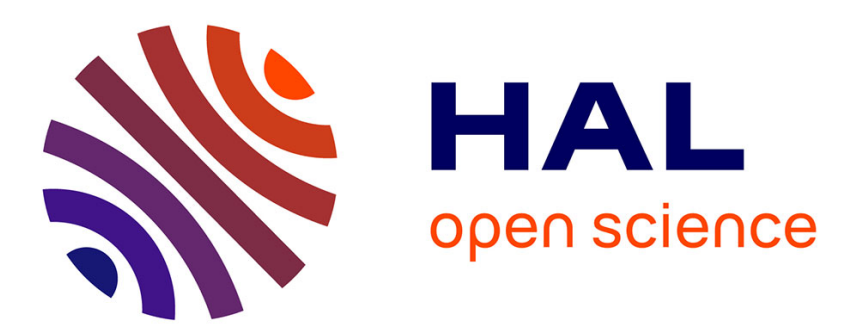

\title{
Nonlinear dynamics of a support-excited flexible rotor with hydrodynamic journal bearings
}

M. Zaki Dakel, Sébastien Baguet, Régis Dufour

\section{To cite this version:}

M. Zaki Dakel, Sébastien Baguet, Régis Dufour. Nonlinear dynamics of a support-excited flexible rotor with hydrodynamic journal bearings. Journal of Sound and Vibration, 2014, 333 (10), pp.2774-2799. 10.1016/j.jsv.2013.12.021 . hal-00935678

\section{HAL Id: hal-00935678 https://hal.science/hal-00935678}

Submitted on 25 Sep 2014

HAL is a multi-disciplinary open access archive for the deposit and dissemination of scientific research documents, whether they are published or not. The documents may come from teaching and research institutions in France or abroad, or from public or private research centers.
L'archive ouverte pluridisciplinaire HAL, est destinée au dépôt et à la diffusion de documents scientifiques de niveau recherche, publiés ou non, émanant des établissements d'enseignement et de recherche français ou étrangers, des laboratoires publics ou privés. 


\title{
Non-linear dynamics of a support-excited flexible rotor with hydrodynamic journal bearings
}

\author{
Mzaki Dakel, Sébastien Baguet, Régis Dufour* \\ Université de Lyon, CNRS, INSA-Lyon, LaMCoS UMR5259, F-69621, France
}

\begin{abstract}
The major purpose of this study is to predict the dynamic behavior of an on-board rotor mounted on hydrodynamic journal bearings in the presence of rigid support movements, the target application being turbochargers of vehicles or rotating machines subject to seismic excitation. The proposed onboard rotor model is based on Timoshenko beam finite elements. The dynamic modeling takes into account the geometric asymmetry of shaft and/or rigid disk as well as the six deterministic translations and rotations of the rotor rigid support. Depending on the type of analysis used for the bearing, the fluid film forces computed with the Reynolds equation are linear/non-linear. Thus the application of the Lagrange's equations yields the linear/non-linear equations of motion of the rotating rotor in bending with respect to the moving rigid support which represents a non-inertial frame of reference. These equations are solved using the implicit Newmark time-step integration scheme. Due to the geometric asymmetry of the rotor and to the rotational motions of the support, the equations of motion include time-varying parametric terms which can lead to lateral dynamic instability. The influence of sinusoidal rotational or translational motions of the support, the accuracy of the linear 8-coefficient bearing model and the interest of the non-linear model for a hydrodynamic journal bearing are examined and discussed by means of stability charts, orbits of the rotor, time history responses, fast Fourier transforms, bifurcation diagrams as well as Poincaré maps.
\end{abstract}

Keywords: Rotordynamics, asymmetric rotor, on-board rotor, finite element method, hydrodynamic journal bearing, support motion, parametric excitation, non-linear dynamics, transient motion, dynamic instability, bifurcation, chaos.

\section{Introduction}

Rotating machines are among the indispensable parts of the modern engineering industries. Due to the nature of the excitations applied to the machine rotors, most of them can be considered as on-board rotors affected mainly by both the mass unbalance and the support motions. Generator, pump, compressor and gas turbine rotors installed in power plants as well as vehicle turbochargers are examples of rotors on moving support. The unavoidable mass unbalance is due to the eccentricity of the center of mass along the rotor axis. The rotor balancing aims at reducing the mass unbalance but is not able to cancel it completely (see Xu et al. [1] and Kang et al. [2]). Rotors can also be subject to the frequent external movements of their support which can increase the flexural vibration of the rotors and create an unstable dynamic behavior.

In order to enhance the performances, the safety factor, the air-gap and the disk-stator gap have to be reduced. Therefore the prediction of dynamic behavior of the rotating machine components must be performed more and more carefully. In this context, the literature comprises numerous books studying a wide variety of phenomena related to the dynamics of symmetric/asymmetric rigid/flexible rotor systems mounted on linear/non-linear elastic bearings in the case of a fixed support. Just few of them can be cited here (Lalanne and Ferraris [3], Genta [4], Bachschmid et al. [5]). Some works observed the instability of parametrically excited systems. Dufour and Berlioz [6] accomplished the study of Berlioz et al. [7] on the dynamics of a drill-string. They analyzed the time-varying parametric equations of motion of the system by employing the Rayleigh-Ritz method, the Floquet theory and the Friedmann approach. The design using a computer opened doors for a technique called "finite element method" to be applied to the rotor modeling and numerous studies contributed to this topic. The real breakthrough in the finite element modeling of a rotor was presented by Nelson and McVaugh [8] who studied a model called "Rayleigh beam" and taking into account the rotary inertia of the shaft and the gyroscopic effects dependent on the speed of rotation of the rotor. Kang et al. [9] modeled rotor-bearing systems using Timoshenko beam finite elements and by taking into account the asymmetry of disk, shaft and/or bearing. They showed that the resonant speeds could change due to various angles between major axes of disk and shaft, the shaft asymmetry as well as the bearing characteristics. Nandi and Neogy [10] proposed an efficient analysis of stability for finite element models of asymmetric rotors and investigated whether an unstable rotor could be stabilized using an isotropic viscous damper. Theoretical and experimental investigations for the isolated and internal resonances of non-linear forced and parametric oscillations of an asymmetric rotor with non-linear spring characteristics were carried out by Ishida et al. [11].

Hydrodynamic bearings play a key role in the design of rotor systems and an accurate prediction of the vibration characteristics of the rotor-bearing systems must be made. Therefore the hydrodynamic bearing performance is predicted and reported in the extant literature by mathematical formulations firmly established. The fluid film forces are strongly non-linear functions of the displacement and velocity of the rotor. Although they act locally on the system, rotordynamics is significantly affected and wholly non-linear.

* Corresponding author. Tel.: +334724382 02; fax: +33472438930.

E-mail address: regis.dufour@insa-lyon.fr (R. Dufour). 
Khonsari and Chang [12] showed that there was a stable region in the short bearing clearance circle outside of which, any initial conditions for the non-linear transient dynamic problem would yield an unstable orbit even if the hydrodynamic bearing should be stable according to the linearized stability analysis. Zhao et al. [13] predicted the linearized stability and presented a comparison between linear and non-linear mass unbalance responses of a flexible rotor-hydrodynamic bearing system modeled by the lump mass method to reduce the degrees of freedom. Li and $\mathrm{Xu}$ [14] studied a Jeffcott rotor supported on oil film infinite-length bearings to obtain periodic orbits, their periods and their stability using the generalized shooting method. Harmonic, sub-harmonic, quasiperiodic and chaotic responses and their Poincaré maps were investigated for a rigid rotor on hydrodynamic short bearings by Brown et al. [15], for a finite element rotor model on hydrodynamic elliptical bearings by Zheng and Hasebe [16] and for a rigid rotor on hydrodynamic elliptical bearings by Shen et al. [17]. Kishor and Gupta [18] used non-linear analytical characteristics for hydrodynamic long bearings and introduced them in a rigid rotor-bearing model with a simplified spur gear model. Baguet and Velex [19] combined a finite element shaft model based on the Timoshenko beam theory with both gear and bearing non-linearities which were represented by spur gear mesh stiffness and hydrodynamic short bearing forces. Baguet and Jacquenot [20] extended the study presented in [19] to include helical gears as well as hydrodynamic finite-length bearings and analyzed parametrically the shaft orbits, the dynamic tooth loading factor as well as the hydrodynamic forces.

Some works focused on the dynamic behavior of a rotor under seismic, random or shock excitations of its support. Srinivasan and Soni [21] studied the effect of spin, support rotation as well as axial force and axial torque on the seismic response of a rotor-bearing system. Samali et al. [22] used the Monte Carlo simulation to simulate the non-stationary earthquake ground motions and to determine the statistics of rotating machinery response. Hori and Kato [23] examined a seismic response of a Jeffcott rotor mounted on oil film bearings to a real seismic wave and investigated its stability. Suarez et al. [24] observed that even for strong rotational inputs, the parametric terms in the equations of motion of a finite element rotor model subject to six components of support excitations could be neglected without affecting its seismic response. Subbiah et al. [25] obtained the amplitude power spectral density due to random excitations of the support for studying the rotor response using modal analysis methods. Lee et al. [26] proposed a finite element rotor model based on the Timoshenko theory by considering a shock excitation of the support and the statespace Newmark method and focused on the experimental behavior of a rotor under this excitation.

The effect of the flexible foundation on rotordynamics was investigated from theoretical and experimental points of view, see, for example, Bonello and Brennan [27]. Da Silva Tuckmantel et al. [28] represented the supporting structure (foundation) of a rotating system by coupled as well as uncoupled modes and tested the methods of mechanical impedance and mixed coordinates to calculate the system responses. The experimental tests of Feng and Hahn [29] showed that even with input data truncated to two significant digits, satisfactory identification were possible for a flexibly supported undamped rigid block foundation in rotating machinery.

Few works are referenced in the literature on the investigation of dynamic behavior of a rotor in the case of a harmonic motion of its support. Duchemin et al. [30] observed the motion stability of a rotor under a sinusoidal rotation of the support by employing the Rayleigh-Ritz and multiple scales methods. They presented also experimental results to validate the analytical study. The work of Driot et al. [31] was based on the model presented in [30], described the numerical orbits of the rotor and compared them with experimental ones. El-Saeidy and Sticher [32] derived the equations of motion of a rigid rotor linear/non-linear bearing system subject to rotating mass unbalance plus harmonic excitations of the support along or around lateral directions. They presented analytical frequency responses in the case of linear bearings, while they discussed numerical results with regards to the time domain, the fast Fourier transform as well as the Poincaré map in the case of a bearing cubic non-linearity. Das et al. [33] investigated the active vibration control of a flexible rotor system modeled by Rayleigh beam finite elements and excited by a mass unbalance as well as a periodic rotational motion of the support. They performed the control with electromagnetic control force provided by an actuator placed on the stator in a suitable plane around the rotor and it was successful for avoiding the lateral parametric instability due to the sinusoidal rotation of the support.

A significant number of previously stated papers treat support-excited rotor systems. Nevertheless, few studies focus on the harmonically excited on-board rotors. Moreover, these papers focus on constructing simplified models. The adoption of simplified models facilitates the understanding of the behavior of such dynamic systems but it is limited in the practical applications and reduces the required accuracy between the theoretical rotor models and the realistic ones under the operating conditions. The most common simplifications and limitations are linked either with the geometry of the rotor (for example, Jeffcott rotor [23]), with the utilization of the Rayleigh-Ritz method instead of the finite element method for the spatial discretization of the rotor model (for example, [30,31]), with the rotors supported by elastic bearings with constant damping and stiffness coefficients (for example, $[32,33]$ ) or with the kind of rotor support motion (simple rotation or translation).

This paper overcomes the previous limitations and contributes to the construction of an improved on-board rotor model. Namely, a rotor with geometric asymmetry due to the shaft and/or the rigid disk is discretized using the finite element method based on the Timoshenko beam theory, mounted on hydrodynamic journal bearings of external forces calculated using the Reynolds equation [34], and excited by combined deterministic motions (three rotations and three translations) of the support. The vibration analysis focuses on the case of bending of the flexible rotor. Eulerian angles are thus employed to describe the orientation of disk and shaft with respect to the moving support. The kinetic and strain energies in addition to the virtual work of the flexible rotor components are computed. Using the short bearing theory in order to obtain the hydrodynamic fluid forces from the Reynolds equation, two models are considered for the hydrodynamic journal bearing: a simplified linearized model with eight constant damping and spring coefficients and a non-linear model where the bearing is treated as external non-linear forces acting on the shaft. The Lagrange's equations are applied to establish the linear/non-linear differential equations of vibratory motion of the rotor in bending with respect to the rigid support representing a non-inertial reference frame. The governing equations of motion display periodic parametric coefficients due to the asymmetry of the rotor and time-varying parametric coefficients due to the support rotations. These parametric coefficients are considered as generators of internal excitation and can create lateral dynamic instability. The equations of motion are solved by the implicit Newmark time-step integration algorithm which must be combined with the Newton-Raphson iterative procedure in the case of a non-linear bearing model. The influence of sinusoidal motions of the support on the dynamics of the onboard rotor-bearing system is analyzed by means of stability charts, orbits of the rotor, time history responses, fast Fourier transforms (FFTs), bifurcation diagrams and Poincaré maps. In addition, a comparison between the linear and non-linear systems is performed to assess the validity range of the linearized 8-coefficient bearing model. 


\section{Problem statement and basic considerations}

Let Fig. 1(a) present the basic components of the rotor: the disk (1), the shaft (2), the mass unbalance (3), the bearing (4) and the support (5). The mass unbalance is distributed continuously as well as in a generic way and cannot completely be canceled. The excitations taken into account are due to the inevitable mass unbalance and to the imposed movements of the support. The transverse deflections of the elastic line of the rotor shaft are studied.

The following assumptions are used: the disk is rigid, the shaft is deformable and modeled by Timoshenko beam elements of constant cross-section solicited in bending along two orthogonal directions, the shaft and/or rigid disk can be asymmetric, the mass unbalance is modeled by concentrated masses, the bearings supporting the shaft are flexible of hydrodynamic short type, the support is rigid and mobile, the rotor rotates at a constant speed $\Omega$.

The consideration of the support motion may modify the form of the equations of motion of a rotor in bending compared to those obtained in the case of a fixed support. In order to develop a modeling as simple as possible, the approach presented by Duchemin et al. [30] is adopted. Three frames of reference are utilized to take into account the movement of the rotor with respect to the support and that of the support with respect to the ground.

The derivation of the equations of motion is inspired from the theory described by Lalanne and Ferraris [3]. First, the coordinate systems needed to describe the motion of an on-board rotor are defined and the vectors expressing the rotations between them are calculated. Then, the kinetic energies for a disk, a shaft element and a concentrated mass unbalance, the strain energy for a shaft element as well as the virtual work for a bearing are calculated. Moreover, the rotating flexible rotor is discretized using the Timoshenko beam finite elements. Finally, the differential equations of motion of an on-board rotor in bending are deduced from the Lagrange's equations being applied with respect to the generalized coordinates $q_{i}$ by

$$
\frac{\mathrm{d}}{\mathrm{d} t}\left(\frac{\partial T}{\partial \dot{q}_{i}}\right)-\frac{\partial T}{\partial q_{i}}+\frac{\partial U}{\partial q_{i}}=F_{q_{i}}
$$

with $n_{\text {dof }}\left(1 \leq i \leq n_{\text {dof }}\right)$ the number of degrees of freedom, $T$ and $U$ the kinetic and strain energies respectively, $F_{q_{i}}$ the external forces corresponding to $q_{i}$. The symbol "." refers to the differentiation with respect to time $t$.

(a)

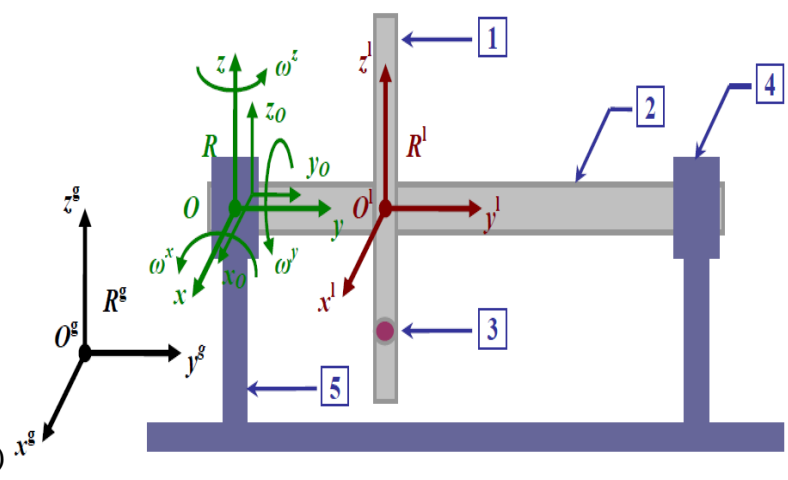

(b) $x$

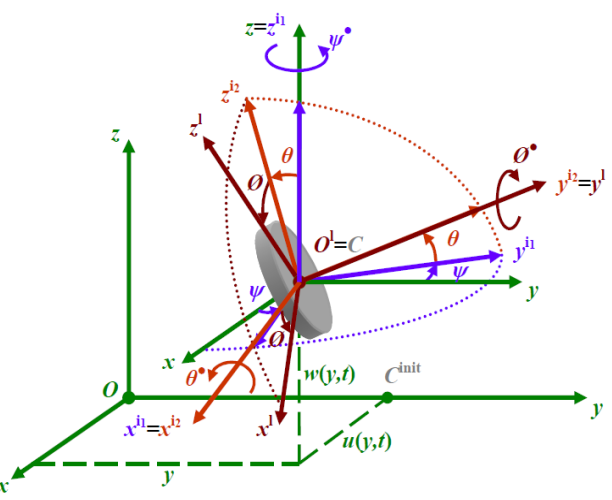

Fig. 1. (a) Components and frames of reference of the on-board rotor model, (b) Euler angles for the transformation between the frame linked with the moving support $R$ and the local frame $R^{1}$.

\section{Kinematics of the on-board rotor}

Three principal Cartesian frames of reference shown in Fig. 1(a) are introduced to take into consideration the movement of the rotor support: $R^{\mathrm{g}}\left(x^{\mathrm{g}}, y^{\mathrm{g}}, z^{\mathrm{g}}\right)$ is the Galilean frame of reference fixed to the ground, $R(x, y, z)$ is the non-inertial frame of reference attached to the moving rigid support, $R^{1}\left(x^{1}, y^{1}, z^{1}\right)$ is the non-inertial local frame of reference (moving with the rotor during its operation) attached to the mass center of the rigid disk or the geometric center of a section of the deformable shaft. The origins of the frames of reference $R^{g}, R$ and $R^{1}$ are $O^{g}, O$ and $O^{1}$ respectively.

The elastic line of the non-deformed shaft is along the $O y$ axis on which the mass center of the disk is located. Moreover $x O y$ and $y O z$ are both planes of symmetry for the disk and the non-deformed shaft. Thus $O x, O y$ and $O z$ are principal axes of inertia.

The translational motions of the rotor support are described by the coordinates $x_{o}(t), y_{O}(t)$ and $z_{o}(t)$ of the position vector $\mathbf{O}^{\mathrm{g}} \mathbf{O}$ projected in the frame attached to the support $R$. The rotational motions of the rotor support are described by the components $\omega^{x}(t)$, $\omega^{y}(t)$ and $\omega^{z}(t)$ of the angular velocity vector $\boldsymbol{\omega}_{R}^{R^{g}}$ of the rigid support $R$ with respect to the ground $R^{g}$ projected in the frame $R$.

In the classical rotordynamics theory, the transformation between the frames $R$ and $R^{1}$ shown in Fig. 1(b) is performed by two intermediate coordinate systems $R^{\mathrm{i}_{1}}\left(x^{\mathrm{i}_{1}}, y^{\mathrm{i}_{1}}, z^{\mathrm{i}_{1}}\right)$ as well as $R^{\mathrm{i}_{2}}\left(x^{\mathrm{i}_{2}}, y^{\mathrm{i}_{2}}, z^{\mathrm{i}_{2}}\right)$ and the Euler angles $\psi(y, t), \theta(y, t), \phi(t)$ which permit describing the angular velocity vector $\boldsymbol{\omega}_{R^{1}}^{R}$ of the rotor $R^{1}$ with respect to its support $R$. The angular velocity vector of the rotor $R^{1}$ with respect to the ground $R^{\mathrm{g}}$ measured in the frame $R^{1}$ is described by

$$
\boldsymbol{\omega}_{R^{1}}^{R^{8}}=\boldsymbol{\omega}_{R}^{R^{8}}+\boldsymbol{\omega}_{R^{1}}^{R}=\left[\omega^{x^{1}}, \omega^{y^{1}}, \omega^{z^{1}}\right]_{R^{1}}^{\mathrm{T}}
$$

where the superscript $\mathrm{T}$ is the matrix transposition symbol. The components $\left(\omega^{x^{1}}, \omega^{y^{1}}, \omega^{z^{1}}\right)$ are formulated as a function of $(\psi, \theta, \phi)$ and their time derivative as well as $\left(\omega^{x}, \omega^{y}, \omega^{z}\right)$. Further details about the kinematics of the on-board rotor can be found in [35]. Let us consider an arbitrary point $C^{\text {init }}$ along the elastic line. Namely, it represents the geometric center of the non-deformed 
shaft. Its coordinates in the frame $R$ are $(0, y, 0)$. Let $u(y, t)$ and $w(y, t)$ be its time-dependent dynamic lateral displacements due to bending along the $O x$ and $O z$ axes, see Fig. 1(b). As its coordinate along the axial axis is assumed to be a constant with respect to the rotor support, the axial displacement along the $O y$ axis is not taken into account.

\section{Energy and virtual work calculations of the on-board rotor-bearing system components}

This section includes formulations for the disk, the shaft, the rotating mass unbalance and the hydrodynamic journal bearing. The kinetic and strain energies are measured by an observer situated at the ground and their terms are written with respect to the frame linked with the rotor rigid support $R$. The rotary inertia, the gyroscopic inertia, the shear deformation of shaft as well as the geometric asymmetry of shaft and/or rigid disk are taken into account.

\subsection{Disk}

According to the assumptions presented in Section 2, the disk is assumed to be rigid and only its kinetic energy $T_{\mathrm{d}}$ is calculated. This energy consists of the scalar sum of the absolute translational kinetic energy and the absolute rotational kinetic energy about the disk mass center located at the generic abscissa $y_{\mathrm{d}}$ along the $O y$ axis, i.e.,

$$
T_{\mathrm{d}}=\frac{m_{\mathrm{d}}}{2}\left(\mathbf{v}_{O_{\mathrm{d}}^{\mathrm{d}}}^{R^{g}}\right)^{\mathrm{T}} \mathbf{v}_{O_{\mathrm{d}}^{\mathrm{d}}}^{R^{g}}+\frac{1}{2}\left(\boldsymbol{\omega}_{R^{1}}^{R^{g}}\right)^{\mathrm{T}} \mathbf{I}_{m_{\mathrm{d}}} \boldsymbol{\omega}_{R^{\prime}}^{R^{g}} \quad \text { with } \quad \mathbf{I}_{m_{\mathrm{d}}}=\operatorname{diag}\left[\begin{array}{ccc}
I_{m_{\mathrm{d}}}^{x} & I_{m_{\mathrm{d}}}^{y} & I_{m_{\mathrm{d}}}^{z}
\end{array}\right]=\operatorname{diag}\left[\begin{array}{lll}
I_{m_{\mathrm{d}}}^{\mathrm{mo}}+I_{m_{\mathrm{d}}}^{\mathrm{di}} & I_{m_{\mathrm{d}}}^{y} & I_{m_{\mathrm{d}}}^{\mathrm{mo}}-I_{m_{\mathrm{d}}}^{\mathrm{di}}
\end{array}\right]
$$

where $m_{\mathrm{d}}$ is the mass of the disk, $\mathbf{v}_{O_{\mathrm{d}}^{1}}^{R^{8}}$ is the translational velocity vector of its center and $\mathbf{I}_{m_{\mathrm{d}}}$ is its principal inertia tensor. $I_{m_{\mathrm{d}}}^{x}$, $I_{m_{\mathrm{d}}}^{y}$ and $I_{m_{\mathrm{d}}}^{z}$ are the principal moments of inertia of the disk mass about the $O x, O y$ and $O z$ axes respectively. In addition, $I_{m_{\mathrm{d}}}^{\mathrm{mo}}$ and $I_{m_{\mathrm{d}}}^{\mathrm{di}}$ are used to seperate the effects due to the mean moment of inertia of the disk mass and those due to the moment of inertia modeling the disk geometric asymmetry. Thus when the disk is asymmetric, the moments of inertia $I_{m_{\mathrm{d}}}^{x}$ as well as $I_{m_{\mathrm{d}}}^{z}$ are different and the contribution of the moment of inertia $I_{m_{\mathrm{d}}}^{\mathrm{di}}$ in them is not nil.

The translational velocity vector $\mathbf{v}_{O_{\mathrm{d}}^{\mathrm{d}}}^{R^{8}}$ is found through the absolute position vector $\mathbf{O}^{\mathrm{g}} \mathbf{O}_{\mathrm{d}}^{1}$ of the disk mass center positioned at the arbitrary abscissa $y_{\mathrm{d}}$ with respect to the frame $R$. The latter is observed from the ground and expressed in the frame fixed to the support $R$ by

$$
\mathbf{O}^{\mathrm{g}} \mathbf{O}_{\mathbf{d}}^{1}=\mathbf{O}^{\mathrm{g}} \mathbf{O}+\mathbf{O O}_{\mathbf{d}}^{\mathbf{1}}=\left[x_{O}, y_{O}, z_{O}\right]_{R}^{\mathrm{T}}+\left[u_{\mathrm{d}}, y_{\mathrm{d}}, w_{\mathrm{d}}\right]_{R}^{\mathrm{T}}=\left[u_{\mathrm{d}}+x_{o}, y_{\mathrm{d}}+y_{O}, w_{\mathrm{d}}+z_{O}\right]_{R}^{\mathrm{T}}
$$

Using the transport theorem relating the time derivative of a vector measured from a fixed frame to that of the same vector but measured from a moving frame, the derivation of the position vector $\mathbf{O}^{\mathbf{g}} \mathbf{O}_{\mathbf{d}}^{1}$ yields

$$
\frac{\mathrm{d} \mathbf{O}^{\mathrm{g}} \mathbf{O}_{\mathrm{d}}^{R^{R^{8}}}}{\mathrm{~d} t}=\frac{\mathrm{d} \mathbf{O}^{\mathrm{g}} \mathbf{O}_{\mathbf{d}}^{1}}{\mathrm{~d} t}+\boldsymbol{\omega}_{R}^{R^{8}} \wedge \mathbf{O}^{\mathrm{g}} \mathbf{O}_{\mathrm{d}}^{\mathbf{1}}=\left[\dot{u}_{O_{\mathrm{d}}^{1}}, \dot{v}_{O_{\mathrm{d}}^{1}}, \dot{w}_{O_{\mathrm{d}}^{1}}\right]_{R}^{\mathrm{T}}=\mathbf{v}_{O_{\mathrm{d}}^{1}}^{R^{g}}
$$

where $\dot{u}_{o_{\mathrm{d}}^{1}}, \dot{v}_{O_{\mathrm{d}}^{1}}$ and $\dot{w}_{o_{\mathrm{d}}^{\prime}}$ are the components of the translational velocity vector of the disk center. Considering the components $\omega_{\mathrm{d}}^{x^{1}}$, $\omega_{\mathrm{d}}^{y^{1}}$ and $\omega_{\mathrm{d}}^{z^{1}}$ of the vector $\boldsymbol{\omega}_{R^{1}}^{R^{8}}$ for the disk, the expression of its kinetic energy becomes

$$
T_{\mathrm{d}}=\frac{m_{\mathrm{d}}}{2}\left(\dot{u}_{O_{\mathrm{d}}^{\mathrm{d}}}^{2}+\dot{v}_{O_{\mathrm{d}}^{\mathrm{d}}}^{2}+\dot{w}_{O_{\mathrm{d}}^{\mathrm{d}}}^{2}\right)+\frac{1}{2}\left(I_{m_{\mathrm{d}}}^{\mathrm{mo}}\left(\omega_{\mathrm{d}}^{\mathrm{x}^{1} 2}+\omega_{\mathrm{d}}^{z^{1} 2}\right)+I_{m_{\mathrm{d}}}^{y} \omega_{\mathrm{d}}^{y^{1} 2}+I_{m_{\mathrm{d}}}^{\mathrm{di}}\left(\omega_{\mathrm{d}}^{x^{1} 2}-\omega_{\mathrm{d}}^{z^{1} 2}\right)\right)
$$

The rotor runs at a constant speed of rotation $\Omega$. So the spinning angle $\phi$ and its time derivative $\dot{\phi}$ are replaced by $\Omega t$ and $\Omega$ respectively. The translational displacements $\left(u_{\mathrm{d}}, w_{\mathrm{d}}\right)$, the angles of rotation $\left(\psi_{\mathrm{d}}, \theta_{\mathrm{d}}\right)$ and their time derivatives are very small. Thus the trigonometric functions are replaced by their Taylor series expansion and the resulting expressions are limited at the order 2 , then

$$
\sin \theta_{\mathrm{d}} \simeq \theta_{\mathrm{d}} \quad ; \quad \cos \theta_{\mathrm{d}} \simeq 1-\frac{\theta_{\mathrm{d}}^{2}}{2} \quad ; \quad \sin \psi_{\mathrm{d}} \simeq \psi_{\mathrm{d}} \quad ; \quad \cos \psi_{\mathrm{d}} \simeq 1-\frac{\psi_{\mathrm{d}}^{2}}{2}
$$

\subsection{Shaft}

The shaft is assumed to be flexible and is modeled by beam elements of constant cross-section and constant moments of inertia. The kinetic energy of the shaft element is obtained by taking a shaft elementary volume similar to a disk of very small thickness dy . Considering the components $\dot{u}_{O^{1}}, \dot{v}_{O^{1}}, \dot{w}_{O^{1}}$ of the vector $\mathbf{v}_{O^{1}}^{R^{8}}$ and the components $\omega^{x^{1}}, \omega^{y^{1}}, \omega^{z^{1}}$ of the vector $\boldsymbol{\omega}_{R^{1}}^{R^{8}}$, the expression for the kinetic energy $T_{\mathrm{sh}}$ of the shaft element, whose end-points have the abscissas $y_{1}$ and $y_{2}$ along the $O y$ axis, is written in the following form

$$
T_{\mathrm{sh}}=\frac{\rho_{\mathrm{sh}} S_{\mathrm{sh}}}{2} \int_{y_{1}}^{y_{2}}\left(\dot{u}_{O^{\prime}}^{2}+\dot{v}_{O^{\prime}}^{2}+\dot{w}_{O^{\prime}}^{2}\right) \mathrm{d} y+\frac{1}{2}\left(\rho_{\mathrm{sh}} I_{S_{\mathrm{sh}}}^{\mathrm{mo}} \int_{y_{1_{1}}}^{y_{2}}\left(\omega^{x^{\prime} 2}+\omega^{z^{1} 2}\right) \mathrm{d} y+2 \rho_{\mathrm{sh}} I_{S_{\mathrm{sh}}}^{\mathrm{mo}} \int_{y_{1}}^{y_{2}} \omega^{y^{\prime} 2} \mathrm{~d} y+\rho_{\mathrm{sh}} I_{S_{\mathrm{sh}}}^{\mathrm{di}} \int_{y_{1}}^{y_{2}}\left(\omega^{x^{t_{2}}}-\omega^{z^{\mathrm{l} 2}}\right) \mathrm{d} y\right)
$$

where $\rho_{\mathrm{sh}}, S_{\mathrm{sh}}$ and $l_{\mathrm{sh}}\left(l_{\mathrm{sh}}=y_{2}-y_{1}\right)$ are respectively the density, the cross-sectional area and the length of the shaft element. $I_{S_{\mathrm{sh}}}^{\mathrm{mo}}=\left(I_{S_{\mathrm{sh}}}^{x}+I_{S_{\mathrm{sh}}}^{z}\right) / 2$ and $I_{S_{\mathrm{sh}}}^{\mathrm{di}}=\left(I_{S_{\mathrm{sh}}}^{x}-I_{S_{\mathrm{sh}}}^{z}\right) / 2$ are respectively the mean moment of inertia of the cross-sectional area and the moment of inertia characterizing the asymmetry of the shaft.

The rigid support motion relative to the ground has no influence on the strain energy of the shaft because the latter depends only on the stresses and therefore on the transverse deflection of the shaft with respect to the rotor support $R$. In addition to the bending deformation, the shear effects introduced by Timoshenko and the second-order (non-linear) terms of the strain field are accounted. The non-linear strains are linked with the centrifugal stiffening being proportional to the square of the support angular velocity 
around the transverse axes and corresponding to the geometric potential energy. Thus the strain energy $U_{\text {sh }}$ of the shaft element is expressed by

$$
\begin{aligned}
U_{\mathrm{sh}}= & \frac{E_{\mathrm{sh}}}{2} I_{S_{\mathrm{sh}}}^{\mathrm{mo}} \int_{y_{1}}^{y_{2}}\left(\left(\frac{\partial \psi}{\partial y}\right)^{2}+\left(\frac{\partial \theta}{\partial y}\right)^{2}\right) \mathrm{d} y+\frac{G_{\mathrm{sh}} \kappa_{\mathrm{sh}}^{\mathrm{mo}} S_{\mathrm{sh}}}{2} \int_{y_{1}}^{y_{2}}\left(\left(\frac{\partial u}{\partial y}+\psi\right)^{2}+\left(\frac{\partial w}{\partial y}-\theta\right)^{2}\right) \mathrm{d} y-\frac{1}{2}\left(E_{\mathrm{sh}} I_{S_{\mathrm{sh}}}^{\mathrm{di}} \int_{y_{1}}^{y_{2}}\left(\left(\frac{\partial \psi}{\partial y}\right)^{2}-\left(\frac{\partial \theta}{\partial y}\right)^{2}\right) \mathrm{d} y\right. \\
& \left.-G_{\mathrm{sh}} \mathrm{sh}_{\mathrm{sh}}^{\mathrm{di}} S_{\mathrm{sh}} \int_{y_{1}}^{y_{2}}\left(\left(\frac{\partial u}{\partial y}+\psi\right)^{2}-\left(\frac{\partial w}{\partial y}-\theta\right)^{2}\right) \mathrm{d} y\right) \cos 2 \Omega t-\left(E_{\mathrm{sh}} I_{S_{\mathrm{sh}}}^{\mathrm{di}} \int_{y_{1}}^{y_{2}} \frac{\partial \psi}{\partial y} \frac{\partial \theta}{\partial y} \mathrm{~d} y\right. \\
& \left.-G_{\mathrm{sh}} \mathrm{sh}_{\mathrm{sh}}^{\mathrm{di}} S_{\mathrm{sh}} \int_{y_{1}}^{y_{2}}\left(\frac{\partial u}{\partial y}+\psi\right)\left(\frac{\partial w}{\partial y}-\theta\right) \mathrm{d} y\right) \sin 2 \Omega t+\frac{\rho_{\mathrm{sh}} S_{\mathrm{sh}}}{4} \int_{y_{1}}^{y_{2}}\left(\left(l_{\mathrm{sh}}+y_{1}\right)^{2}-y^{2}\right)\left(\left(\frac{\partial u}{\partial y}\right)^{2}+\left(\frac{\partial w}{\partial y}\right)^{2}\right) \mathrm{d} y\left(\omega^{x 2}+\omega^{22}\right)
\end{aligned}
$$

where $E_{\mathrm{sh}}, G_{\mathrm{sh}}=E_{\mathrm{sh}} / 2\left(1+v_{\mathrm{sh}}\right)$ and $v_{\mathrm{sh}}$ are respectively the Young's modulus, the shear modulus and the Poisson's ratio of the isotropic shaft material. $\kappa_{\mathrm{sh}}^{\mathrm{mo}}=\left(\kappa_{\mathrm{sh}}^{\mathrm{x}}+\kappa_{\mathrm{sh}}^{z}\right) / 2$ and $\kappa_{\mathrm{sh}}^{\mathrm{di}}=\left(\kappa_{\mathrm{sh}}^{\mathrm{x}}-\kappa_{\mathrm{sh}}^{z}\right) / 2$ are respectively the mean shear correction factor of the crosssectional area and that relative to the section asymmetry of the shaft.

\subsection{Mass unbalance}

The mass unbalance is modeled by a concentrated mass $m_{\mathrm{mu}}$ placed at a point $P_{\mathrm{mu}}$ with a distance $r_{\mathrm{mu}}$ from the geometric center of the cross-sectional area of the shaft. Its initial angle with the $O z$ axis of the frame $R$ at rest is $\eta_{\mathrm{mu}}$. The mass unbalance remains in a plane perpendicular to the $O y$ axis with a constant abscissa $y_{\text {mu }}$ along the $O y$ axis relative to the rotor support $R$. Its kinetic energy $T_{\mathrm{mu}}$ is defined as follows

$$
T_{\mathrm{mu}}=\frac{m_{\mathrm{mu}}}{2}\left(\mathbf{v}_{P_{\mathrm{mu}}}^{R^{g}}\right)^{\mathrm{T}} \mathbf{v}_{P_{\mathrm{mu}}}^{R^{8}}
$$

The translational velocity vector $\mathbf{v}_{P_{\mathrm{mu}}}^{R^{g}}$ is deduced from the absolute position vector $\mathbf{O}^{g} \mathbf{P}_{\mathrm{mu}}$ of the mass unbalance. The latter is measured from the ground and written relative to the frame linked with the support $R$ as

$$
\mathbf{O}^{\mathrm{g}} \mathbf{P}_{\mathrm{mu}}=\mathbf{O}^{\mathrm{g}} \mathbf{O}+\mathbf{O} \mathbf{O}_{\mathrm{mu}}^{1}+\mathbf{O}_{\mathrm{mu}}^{1} \mathbf{P}_{\mathrm{mu}}=\left[u_{\mathrm{mu}}+x_{O}+r_{\mathrm{mu}} \sin \left(\Omega t+\eta_{\mathrm{mu}}\right), y_{\mathrm{mu}}+y_{O}, w_{\mathrm{mu}}+z_{O}+r_{\mathrm{mu}} \cos \left(\Omega t+\eta_{\mathrm{mu}}\right)\right]_{R}^{\mathrm{T}}
$$

Applying the transport theorem, the time derivative of the position vector $\mathbf{O}^{\mathrm{g}} \mathbf{P}_{\mathrm{mu}}$ is of the following form

$$
\frac{\mathrm{d} \mathbf{O}^{\mathrm{g}} \mathbf{P}_{\mathrm{mu}}{ }^{R^{g}}}{\mathrm{~d} t}=\frac{\mathrm{d} \mathbf{O}^{\mathrm{g}} \mathbf{P}_{\mathbf{m u}}{ }^{R}}{\mathrm{~d} t}+\boldsymbol{\omega}_{R}^{R^{g}} \wedge \mathbf{O}^{\mathrm{g}} \mathbf{P}_{\mathrm{mu}}=\mathbf{v}_{P_{\mathrm{mu}}}^{R^{g}}
$$

The external force vectors obtained by the application of the Lagrange's equations to the kinetic energy of the mass unbalance contain the so-called "centripetal and centrifugal force vectors", sinusoidal tangential force vectors and sinusoidal force vectors fixed in space with respect to the support $R$. Thus in the presence of sinusoidal rotations of the support, the excitation due to the mass unbalance combined with the support rotations is sinusoidal and has not only a frequency equal to the speed of rotation $\Omega$ of the rotor but also a combination of frequencies of speed of rotation of the rotor and support rotation.

\subsection{Hydrodynamic journal bearing}

Fig. 2(a) shows a simple representation of a hydrodynamic bearing which consists of a fixed journal (1) including a rotating shaft (2) and a lubricant (3) separating these two components. The points $O$ and $O^{1}$ are respectively the bearing center and the shaft geometric center. The radius, length and clearance of the bearing are respectively $r_{\mathrm{be}}, l_{\mathrm{be}}$ and $c_{\mathrm{be}}=r_{\mathrm{be}}-r_{\mathrm{sh}}$ where $r_{\mathrm{sh}}$ is the shaft radius.

At a constant speed of rotation $\Omega$ of the rotor and for a constant static load $W_{\mathrm{r}}$ created by the rotor weight, the shaft geometric center $O^{1}$ in the bearing occupies a static equilibrium position $C_{\mathrm{be}}^{0}$ defined by the displacement vector $\boldsymbol{\delta}_{\mathrm{be}}^{0}=\left[u_{\mathrm{be}}^{0}, w_{\mathrm{be}}^{0}\right]_{R}^{\mathrm{T}}$ expressed in the frame $R$ or equivalently by the static eccentricity $e_{\mathrm{be}}^{0}=\left\|\boldsymbol{\delta}_{\mathrm{be}}^{0}\right\|$ of the shaft center in the journal and the static attitude angle $\varphi_{\mathrm{be}}^{0}$ between the $W_{\mathrm{r}}$ load direction and the line of centers $\mathbf{O C}_{\mathrm{be}}^{\mathbf{0}}$.

In the dynamic regime, the fluid film pressure distribution in the hydrodynamic bearing is governed by the Reynolds equation and its integration over the bearing yields the external hydrodynamic forces $\mathbf{F}_{\mathrm{be}}=\left[F_{\mathrm{be}}^{u}, F_{\mathrm{be}}^{w}\right]_{R}^{\mathrm{T}}$ acting on the shaft and projected in the Cartesian frame connected to the rotor support $R$. Denoting the dynamic variation of shaft center position in the bearing (i.e., the shaft center describes an orbit situated within a circle whose radius is equal to the radial clearance of the bearing) due to the dynamic variation of forces applied to the rotor (mass unbalance, fluid film forces and support excitations), the dynamic transverse displacement vector of the shaft center is $\boldsymbol{\delta}_{\mathrm{be}}=\left[u_{\mathrm{be}}, w_{\mathrm{be}}\right]_{R}^{\mathrm{T}}$. The dynamic polar coordinates of the shaft center in the bearing expressed in the frame $R^{\text {be }}(t a, r a)$ are the relative dynamic eccentricity $\varepsilon_{\text {be }}$ (relative dynamic radial displacement) and the dynamic attitude angle $\varphi_{\mathrm{be}}$, see Fig. 2(a). These coordinates and their time derivatives are expressed as

$$
\varepsilon_{\mathrm{be}}=\frac{e_{\mathrm{be}}}{c_{\mathrm{be}}}=\frac{\sqrt{u_{\mathrm{be}}^{2}+w_{\mathrm{be}}^{2}}}{c_{\mathrm{be}}} ; \tan \varphi_{\mathrm{be}}=\frac{u_{\mathrm{be}}}{w_{\mathrm{be}}} ; \quad \dot{\varepsilon}_{\mathrm{be}}=\frac{\dot{u}_{\mathrm{be}} u_{\mathrm{be}}+\dot{w}_{\mathrm{be}} w_{\mathrm{be}}}{c_{\mathrm{be}}^{2} \varepsilon_{\mathrm{be}}} ; \quad \dot{\varphi}_{\mathrm{be}}=\frac{\dot{u}_{\mathrm{be}} w_{\mathrm{be}}-\dot{w}_{\mathrm{be}} u_{\mathrm{be}}}{c_{\mathrm{be}}^{2} \varepsilon_{\mathrm{be}}^{2}}
$$

In the present paper, the short bearing theory is considered (i.e., $l_{\mathrm{be}} / d_{\mathrm{be}} \leq 1 / 8$ where $d_{\mathrm{be}}=2 r_{\mathrm{be}}$ ). Assuming an isothermal and laminar flow of an isoviscous incompressible fluid, the dynamic polar (tangential and radial) components of the hydrodynamic force 
vector can be analytically deduced from the integration of the hydrodynamic fluid pressure field based on the Reynolds equation using the Gümbel boundary conditions which discard the negative pressure from the force integral. These dynamic components in the polar frame linked with the hydrodynamic bearing $R^{\text {be }}$ are defined by the following formulations [34]

$$
F_{\mathrm{be}}^{t a}=\frac{\mu_{\mathrm{be}} r_{\mathrm{be}} l_{\mathrm{be}}^{3} \varepsilon_{\mathrm{be}}}{2 c_{\mathrm{be}}^{2}\left(1-\varepsilon_{\mathrm{be}}^{2}\right)^{2}}\left(4 \dot{\varepsilon}_{\mathrm{be}}+\frac{\pi}{2} \sqrt{1-\varepsilon_{\mathrm{be}}^{2}}\left(\Omega-2 \dot{\varphi}_{\mathrm{be}}\right)\right) ; \quad F_{\mathrm{be}}^{r a}=-\frac{\mu_{\mathrm{be}} r_{\mathrm{be}} l_{\mathrm{be}}^{3}}{2 c_{\mathrm{be}}^{2}\left(1-\varepsilon_{\mathrm{be}}^{2}\right)^{2}}\left(\frac{\pi \dot{\varepsilon}_{\mathrm{be}}\left(1+2 \varepsilon_{\mathrm{be}}^{2}\right)}{\sqrt{1-\varepsilon_{\mathrm{be}}^{2}}}+2 \varepsilon_{\mathrm{be}}^{2}\left(\Omega-2 \dot{\varphi}_{\mathrm{be}}\right)\right)
$$

where $\mu_{\mathrm{be}}$ is the fluid film dynamic viscosity. The equation calculating the static solution can be reached by setting the time derivatives of $\varepsilon_{\mathrm{be}}$ and $\varphi_{\mathrm{be}}$ in Eq. (14) to zero and by setting the magnitude of the vector sum for the force vector components in Eq. (14) to be equal to the static load $W_{\mathrm{r}}$. Thus the resulting non-linear equation is solved by an iterative Newton-Raphson method and provides the relative static eccentricity $\varepsilon_{\mathrm{be}}^{0}$ and then the static radial displacement $e_{\mathrm{be}}^{0}$ of the shaft center in the bearing. The components of the static equilibrium position vector $\boldsymbol{\delta}_{\mathrm{be}}^{0}$ and those of the dynamic fluid force vector $\mathbf{F}_{\mathrm{be}}$ are obtained by a classical change of basis (see Fig. 2(a)) given as

$$
\boldsymbol{\delta}_{\mathrm{be}}^{0}=-\left(\mathbf{R}_{R^{\mathrm{bc}}}^{R}\right)^{\mathrm{T}}\left\{\begin{array}{c}
0 \\
e_{\mathrm{be}}^{0}
\end{array}\right\}_{R^{\mathrm{bc}}} \quad ; \quad \mathbf{F}_{\mathrm{be}}=-\left(\mathbf{R}_{R^{\mathrm{bc}}}^{R}\right)^{\mathrm{T}}\left\{\begin{array}{l}
F_{\mathrm{be}}^{t a} \\
F_{\mathrm{be}}^{r a}
\end{array}\right\}_{R^{\mathrm{bc}}} ; \quad \mathbf{R}_{R^{\mathrm{be}}}^{R}=\left[\begin{array}{cc}
\cos \varphi_{\mathrm{be}}^{0} & -\sin \varphi_{\mathrm{be}}^{0} \\
\sin \varphi_{\mathrm{be}}^{0} & \cos \varphi_{\mathrm{be}}^{0}
\end{array}\right] ; \quad \mathbf{R}_{R^{\mathrm{be}^{\mathrm{be}}}}^{R}=\left[\begin{array}{cc}
\cos \varphi_{\mathrm{be}} & -\sin \varphi_{\mathrm{be}} \\
\sin \varphi_{\mathrm{be}} & \cos \varphi_{\mathrm{be}}
\end{array}\right]
$$

where $\mathbf{R}_{R^{\text {be }}}^{R}$ and $\mathbf{R}_{R^{\text {be }}}^{R}$ are the rotation matrices of the polar frame $R^{\text {be }}$ with respect to $R$ in the static and dynamic cases respectively. According to Eqs. (13)-(15), since the hydrodynamic force vector components depend non-linearly on the dynamic polar coordinates and on their time derivatives, they are non-linear functions of the dynamic transverse displacement and velocity vectors $\left(\boldsymbol{\delta}_{\mathrm{be}}, \dot{\boldsymbol{\delta}}_{\mathrm{be}}\right)$. As the bearing contribution appears as forces $\mathbf{F}_{\mathrm{be}}$ external to the rotor, their virtual work $\delta W_{\mathrm{be}}$ must be established to apply the Lagrange's equations

$$
\delta W_{\text {be }}=\mathbf{F}_{\text {be }}^{\mathrm{T}}\left(\boldsymbol{\delta}_{\text {be }}, \dot{\boldsymbol{\delta}}_{\text {be }}\right) \delta \boldsymbol{\delta}_{\text {be }}
$$

If the dynamic displacements $\boldsymbol{\delta}_{\mathrm{be}}$ of the shaft elastic line are assumed to be small in the vicinity of the static position $\boldsymbol{\delta}_{\mathrm{be}}^{0}$, the linear analysis of a bearing is applied by constructing a first-order Taylor expansion of the dynamic fluid forces $\mathbf{F}_{\mathrm{be}}\left(\boldsymbol{\delta}_{\mathrm{be}}, \dot{\boldsymbol{\delta}}_{\mathrm{be}}\right)$ in the vicinity of the static hydrodynamic forces $\mathbf{F}_{\mathrm{be}}\left(\boldsymbol{\delta}_{\mathrm{be}}^{0}, \mathbf{0}\right)$ as follows

$$
\mathbf{F}_{\text {be }}\left(\boldsymbol{\delta}_{\text {be }}, \dot{\boldsymbol{\delta}}_{\text {be }}\right)=\mathbf{F}_{\text {be }}\left(\boldsymbol{\delta}_{\text {be }}^{0}, \mathbf{0}\right)-\mathbf{c}_{\text {be }} \Delta \dot{\boldsymbol{\delta}}_{\text {be }}-\mathbf{k}_{\text {be }} \Delta \boldsymbol{\delta}_{\text {be }}
$$

with

$$
\mathbf{c}_{\mathrm{be}}=-\left.\frac{\partial \mathbf{F}_{\mathrm{be}}}{\partial \dot{\boldsymbol{\delta}}_{\mathrm{be}}}\right|_{\left(\delta_{\mathrm{be}}^{0}, \mathbf{0}\right)}=\left[\begin{array}{cc}
c_{\mathrm{be}}^{x x} & c_{\mathrm{be}}^{x z} \\
c_{\mathrm{be}}^{z x} & c_{\mathrm{be}}^{z z}
\end{array}\right] ; \quad \mathbf{k}_{\mathrm{be}}=-\left.\frac{\partial \mathbf{F}_{\mathrm{be}}}{\partial \boldsymbol{\delta}_{\mathrm{be}}}\right|_{\left(\hat{\mathrm{o}}_{\mathrm{be}}^{0}, 0\right)}=\left[\begin{array}{cc}
k_{\mathrm{be}}^{x x} & k_{\mathrm{be}}^{x z} \\
k_{\mathrm{be}}^{z x} & k_{\mathrm{be}}^{z z}
\end{array}\right] ; \quad \Delta \boldsymbol{\delta}_{\mathrm{be}}=\boldsymbol{\delta}_{\mathrm{be}}-\boldsymbol{\delta}_{\mathrm{be}}^{0} \quad ; \quad \boldsymbol{\Delta} \dot{\boldsymbol{\delta}}_{\mathrm{be}}=\dot{\boldsymbol{\delta}}_{\mathrm{be}}
$$

The bearing force vector $\mathbf{F}_{\mathrm{be}}\left(\boldsymbol{\delta}_{\mathrm{be}}^{0}, \mathbf{0}\right)$ is related to the static displacement vector $\boldsymbol{\delta}_{\mathrm{be}}^{0}$ and must equilibrate the constant load $W_{\mathrm{r}}$ imposed by the rotor weight. The damping and stiffness matrices $\mathbf{c}_{\mathrm{be}}, \mathbf{k}_{\mathrm{be}}$ of the linearized 8-coefficient hydrodynamic bearing (i.e., four damping and four stiffness coefficients) (see Fig. 2(b)) depend only on the static position $\boldsymbol{\delta}_{\mathrm{be}}^{0}$. Actually, they are easier to obtain in the polar frame $R^{\text {be }}$, i.e., $\mathbf{c}_{\mathrm{be}}^{\mathrm{pe}^{\mathrm{be}}}$ and $\mathbf{k}_{\mathrm{be}}^{\mathrm{k}^{\mathrm{be}}}$. Their analytical expressions can be found in [34] and deduced from Eq. (14). In addition, these expressions are presented in Appendix A. Lastly, it is necessary to execute the transformation between the frames $R^{\text {be }}$ and $R$ in the static condition to produce the matrices $\mathbf{c}_{\mathrm{be}}$ and $\mathbf{k}_{\mathrm{be}}$ in $R$ as follows

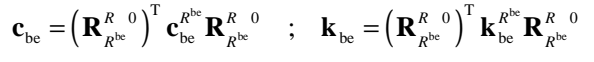

(a)

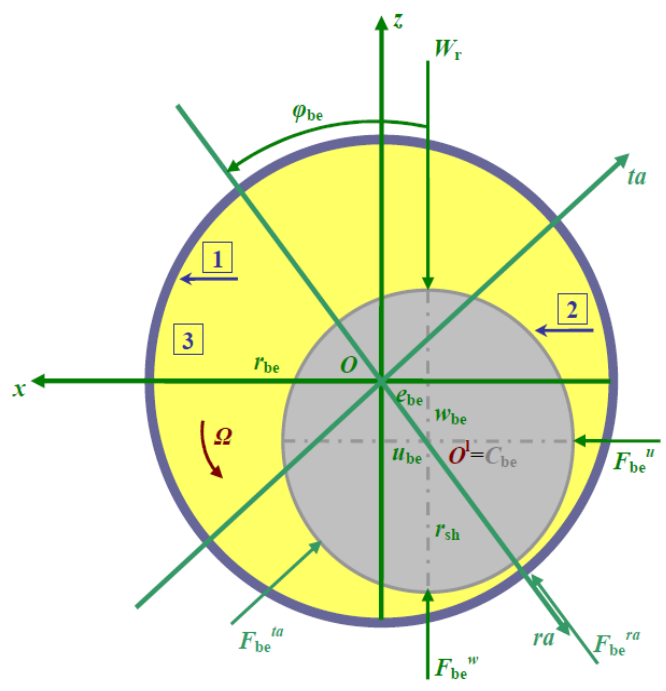

(b)

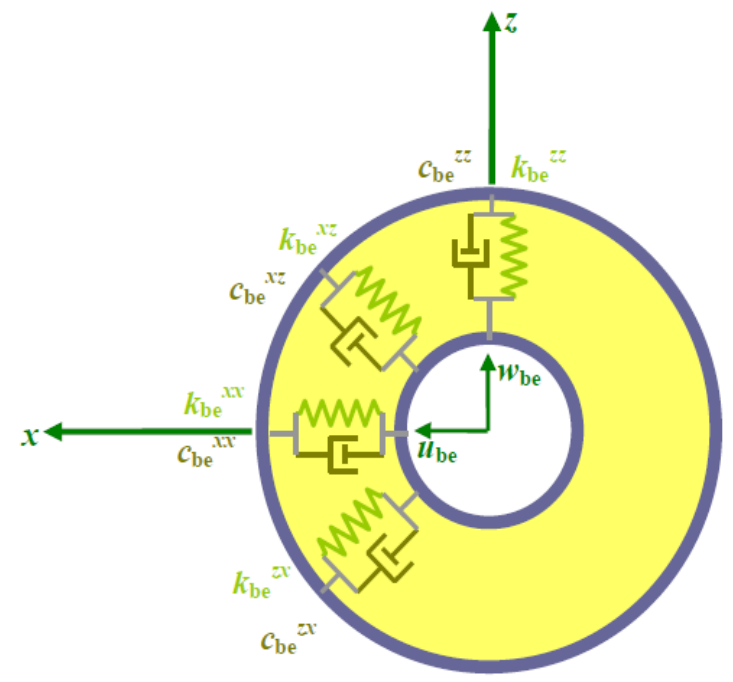

Fig. 2. (a) Schematic diagram of a hydrodynamic bearing, (b) Linearized damping and stiffness coefficients of a bearing model. 


\section{Overall dynamic behavior of the on-board rotor-hydrodynamic bearing system}

\subsection{Finite element formulation}

The finite element method is chosen to discretize the on-board rotor in bending. Each node of nodal interpolation includes four degrees of freedom (two transverse translations and two rotations). The disk, the mass unbalance and the hydrodynamic journal bearing are modeled by one-node finite elements situated at the geometric center of the cross-sectional area of the shaft. On the other hand, the shaft is modeled by $n_{\text {esh }}$ two-node beam finite elements based on the Timoshenko beam theory and thus has $n_{\text {esh }}+1$ nodes. Let us consider a non-deformed shaft finite element $e s h_{i}$ which consists of two nodes $n_{i}$ and $n_{i+1}$ of coordinates $y_{\mathrm{sh}}^{n_{i}}$ and $y_{\mathrm{sh}}^{n_{i+1}}$ along the $O y$ axis. This finite element is of density $\rho_{e s h_{h}}$, cross-sectional area $S_{e s h_{i}}$, length $l_{e s h_{i}}$, moments of inertia $I_{S_{e s s_{i}}}^{\mathrm{mo}}$ and $I_{S_{e s h_{i}}}^{\mathrm{di}}$, Young's modulus $E_{e s h_{i}}$, Poisson's ratio $v_{e s h_{i}}$ as well as shear correction factors $\kappa_{e s h_{i}}^{\mathrm{mo}}$ and $\kappa_{e s h_{i}}^{\mathrm{di}}$ where $i\left(1 \leq i \leq n_{\text {esh }}\right)$ is the shaft finite element number. Its nodal displacement vector $\boldsymbol{\delta}_{e s h_{3}}^{\mathrm{n}}$ projected in the coordinate system attached to the rotor rigid support $R$ is defined by

$$
\boldsymbol{\delta}_{e s h_{i}}^{\mathrm{n}}=\left[u_{e s h_{i}}^{n_{i}}, w_{e s h_{i}}^{n_{i}}, \theta_{e s h_{i}}^{n_{i}}, \psi_{e s h_{i}}^{n_{i}}, u_{e s h_{i}}^{n_{i+1}}, w_{e s h_{i}}^{n_{i+1}}, \theta_{e s h_{i}}^{n_{i+1}}, \psi_{e s h_{i}}^{n_{i+1}}\right]_{R}^{\mathrm{T}}
$$

The above vector is separated into two vectors $\boldsymbol{\delta}_{e s h_{i}}^{\mathrm{n}, u}$ and $\boldsymbol{\delta}_{e s h_{i}}^{\mathrm{n}, w}$ associated with the motions of the nodes $n_{i}$ and $n_{i+1}$ in the planes $x O y$ and $y O z$, then

$$
\boldsymbol{\delta}_{e s h_{i}}^{\mathrm{n}, u}=\left[u_{e s h_{i}}^{n_{i}}, \psi_{e s h_{i}}^{n_{i}}, u_{e s h_{i}}^{n_{i+1}}, \psi_{e s h_{i}}^{n_{i+1}}\right]_{R}^{\mathrm{T}} \quad ; \quad \boldsymbol{\delta}_{e s h_{i}}^{\mathrm{n}, w}=\left[w_{e s h_{i}}^{n_{i}}, \theta_{e s h_{i}}^{n_{i}}, w_{e s h_{i}}^{n_{i+1}}, \theta_{e s h_{i}}^{n_{i+1}}\right]_{R}^{\mathrm{T}}
$$

The dynamic transverse displacements $u_{e s h_{i}}$ and $w_{e s h_{i}}$ of any point $C^{\text {init }}$ due to bending respectively along the $O x$ and $O z$ axes of the frame $R$ as well as the corresponding rotations $\theta_{e s h_{i}}$ and $\psi_{\text {esh }}$ respectively around the $O x$ and $O z$ directions are formulated as a function of the nodal displacement vectors $\boldsymbol{\delta}_{e s h_{i}}^{\mathrm{n}, u}$ and $\boldsymbol{\delta}_{e s h_{i}}^{\mathrm{n}, w}$ in what follows

$$
u_{e s h_{i}}=\overline{\mathbf{F}_{e s h_{i}}^{u}}(\bar{y}) \boldsymbol{\delta}_{e s h_{i}}^{\mathrm{n}, u} ; \psi_{e s h_{i}}=\overline{\mathbf{F}_{e s h_{i}}^{\psi}}(\bar{y}) \boldsymbol{\delta}_{e s h_{i}}^{\mathrm{n}, u} ; w_{e s h_{i}}=\overline{\bar{F}_{e s h_{i}}^{w}}(\bar{y}) \boldsymbol{\delta}_{e s h_{i}}^{\mathrm{n}, w} ; \theta_{e s h_{i}}=\overline{\mathbf{F}_{e s h_{i}}^{\theta}}(\bar{y}) \boldsymbol{\delta}_{e s h_{i}}^{\mathrm{n}, w}
$$

where $\overline{\mathbf{F}_{\text {esh }}^{u}}(\bar{y}), \overline{\mathbf{F}_{\text {esh }}^{\psi}}(\bar{y}), \overline{\mathbf{F}_{\text {esh }}^{w}}(\bar{y})$ and $\overline{\mathbf{F}_{\text {esh }}^{\theta}}(\bar{y})$ are the shape function vectors of a Timoshenko beam finite element. They are thirddegree and second-degree polynomial equations, functions of the dimensionless variable $\bar{y}\left(\bar{y}=y / l_{\text {esh }}\right)$ along the $O y$ axis of the frame $R$ and include the shear effects (see Chen and $\mathrm{Ku}[36]$ ).

\subsection{Equations of motion}

The Lagrange's equations are applied to the energies for the disk, the shaft finite element and the mass unbalance (see Eqs. (6), (8), (9) and (10)) as well as to the virtual work of the hydrodynamic bearing (see Eq. (16)) and the resulting elementary matrices and vectors are appropriately assembled. Due to the presence of hydrodynamic bearings, the boundary conditions do not exist and the corresponding row and column entries of the assembled matrices and vectors are not eliminated. Thus the obtained global matrices and vectors are respectively of dimension $n_{\mathrm{g}} \times n_{\mathrm{g}}$ and $n_{\mathrm{g}} \times 1$ where $n_{\mathrm{g}}=4\left(n_{\mathrm{esh}}+1\right)$ is the total number of degrees of freedom. The obtained non-linear second-order differential equations describing the dynamic behavior of the on-board rotor-bearing system in bending are written with respect to the non-inertial frame connected to the rigid support $R$ as follows

$$
\mathbf{M}_{\mathrm{r}}(t) \ddot{\boldsymbol{\delta}}_{\mathrm{r}}+\mathbf{C}_{\mathrm{r}}(t) \dot{\boldsymbol{\delta}}_{\mathrm{r}}+\mathbf{K}_{\mathrm{r}}(t) \boldsymbol{\delta}_{\mathrm{r}}=\mathbf{F}_{\mathrm{r}}(t)+\mathbf{F}_{\mathrm{be}}\left(\boldsymbol{\delta}_{\mathrm{r}}, \dot{\boldsymbol{\delta}}_{\mathrm{r}}\right)
$$

where $\mathbf{M}_{\mathrm{r}}(t), \mathbf{C}_{\mathrm{r}}(t)$ and $\mathbf{K}_{\mathrm{r}}(t)$ are the global mass, damping and stiffness matrices with periodic and time-varying parametric terms due to the geometric asymmetry of the rotating rotor and to the rotations of its rigid support respectively. These matrices are not affected by the translational motions of the support. $\ddot{\boldsymbol{\delta}}_{\mathrm{r}}, \dot{\boldsymbol{\delta}}_{\mathrm{r}}$ and $\boldsymbol{\delta}_{\mathrm{r}}$ are the global acceleration, velocity and displacement vectors conforming to the connectivity of the finite elements. $\mathbf{F}_{\mathrm{r}}(t)$ is the global external force vector containing the excitations due to the influence of the mass unbalance as well as to that of the rotational and translational motions of the support. Lastly, the external nonlinear hydrodynamic force vector $\mathbf{F}_{\mathrm{be}}\left(\boldsymbol{\delta}_{\mathrm{r}}, \dot{\boldsymbol{\delta}}_{\mathrm{r}}\right)$ of the bearing is opposite to the rotor motion.

The matrices $\mathbf{M}_{\mathrm{r}}(t), \mathbf{C}_{\mathrm{r}}(t)$ and $\mathbf{K}_{\mathrm{r}}(t)$ mentioned in Eq. (23) are defined as follows

$$
\begin{aligned}
& \mathbf{M}_{\mathrm{r}}(t)=\mathbf{M}_{\mathrm{d}, \mathrm{sh}}+\mathbf{M}_{\mathrm{d}, \mathrm{sh}}^{\mathrm{c}_{2}} \cos 2 \Omega t+\mathbf{M}_{\mathrm{d}, \mathrm{sh}}^{\mathrm{s}_{2}} \sin 2 \Omega t \\
& \mathbf{C}_{\mathrm{r}}(t)=\mathbf{C}_{\mathrm{d}, \mathrm{sh}}^{\mathrm{g}} \Omega+\mathbf{C}_{\mathrm{d}, \mathrm{sh}}^{\mathrm{g}, \mathrm{c}_{2}} \Omega \cos 2 \Omega t+\mathbf{C}_{\mathrm{d}, \mathrm{sh}}^{\mathrm{g}, s_{2}} \Omega \sin 2 \Omega t+\mathbf{C}_{\mathrm{d}, \mathrm{sh}, \mathrm{su}}^{\mathrm{re}, \omega^{y}} \omega^{v} \\
& \mathbf{K}_{\mathrm{r}}(t)=\mathbf{K}_{\mathrm{sh}}^{\mathrm{e}}+\mathbf{K}_{\mathrm{sh}}^{\mathrm{e}, \mathrm{c}_{2}} \cos 2 \Omega t+\mathbf{K}_{\mathrm{sh}}^{\mathrm{e}, s_{2}} \sin 2 \Omega t
\end{aligned}
$$

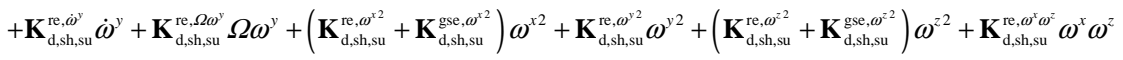

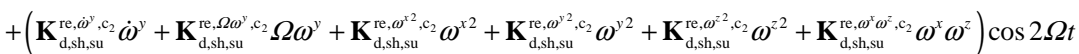

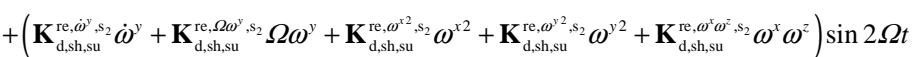

The subscripts "d", "sh" and "su" refer to the disk, the shaft as well as the support respectively and express the contribution to the phenomena represented by the corresponding matrix. The superscripts " $c_{2}$ " and " $s_{2}$ " denote the geometric asymmetry of the rotor expressed in terms of the time-varying trigonometric functions $\cos 2 \Omega t$ and $\sin 2 \Omega t$. The superscript " $\mathrm{g}$ " stands for the rotor gyroscopic effect, "e" for the shaft elasticity corresponding to the bending and shear deformations, "re" for the rotational effects 
due to the support rotations (these effects come from the kinetic energies of the disk and the shaft) and "gse " for the geometric stiffening effects corresponding to the centrifugal stress due to the support rotations (these effects come from the strain energy of the shaft).

The force vector $\mathbf{F}_{\mathrm{r}}(t)$ indicated in Eq. (23) is expressed as follows

with

$$
\mathbf{F}_{\mathrm{r}}(t)=\mathbf{F}_{\mathrm{d}, \mathrm{sh}}^{W_{\mathrm{r}}}+\mathbf{F}_{\mathrm{mu}}(t)+\mathbf{F}_{\mathrm{mu}, \text { su }}(t)+\mathbf{F}_{\mathrm{d}, \mathrm{sh}, \mathrm{su}}(t)+\mathbf{F}_{\mathrm{d}, \mathrm{sh}, \mathrm{su}}^{\mathrm{c}_{2}}(t) \cos 2 \Omega t+\mathbf{F}_{\mathrm{d}, \mathrm{sh}, \mathrm{su}}^{\mathrm{s}_{2}}(t) \sin 2 \Omega t
$$

$$
\begin{aligned}
& \mathbf{F}_{\mathrm{d}, \mathrm{sh}}^{W_{\mathrm{r}}}=-\mathbf{V}_{\mathrm{d}, \mathrm{sh}}^{W_{\mathrm{r}}} W_{\mathrm{r}} \quad ; \quad \mathbf{F}_{\mathrm{mu}}(t)=\mathbf{V}_{\mathrm{mu}}^{\mathrm{c}_{\mathrm{s}}} \Omega^{2} \cos \Omega t+\mathbf{V}_{\mathrm{mu}}^{\mathrm{s}_{1}} \Omega^{2} \sin \Omega t
\end{aligned}
$$

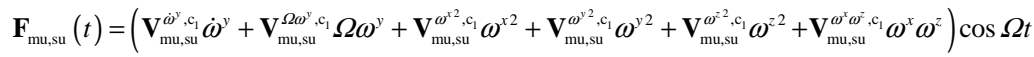

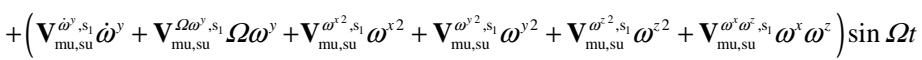

$$
\begin{aligned}
& \mathbf{F}_{\mathrm{d}, \mathrm{sh}, \mathrm{su}}(t)=-\mathbf{V}_{\mathrm{d}, \mathrm{sh}, \mathrm{su}}^{u}\left(\ddot{x}_{O}+2 \dot{z}_{O} \omega^{y}-2 \dot{y}_{O} \omega^{z}+z_{O}\left(\dot{\omega}^{y}+\omega^{x} \omega^{z}\right)-y_{O}\left(\dot{\omega}^{z}-\omega^{x} \omega^{y}\right)-x_{O}\left(\omega^{y 2}+\omega^{z 2}\right)\right) \\
& -\mathbf{V}_{\mathrm{d}, \mathrm{sh}, \mathrm{su}}^{w}\left(\ddot{z}_{O}+2 \dot{y}_{O} \omega^{x}-2 \dot{x}_{O} \omega^{y}+y_{O}\left(\dot{\omega}^{x}+\omega^{y} \omega^{z}\right)-x_{O}\left(\dot{\omega}^{y}-\omega^{x} \omega^{z}\right)-z_{o}\left(\omega^{x 2}+\omega^{y 2}\right)\right) \\
& -\mathbf{V}_{\mathrm{d}, \mathrm{sh}, \mathrm{su}}^{\mathrm{yw}}\left(\dot{\omega}^{x}+\omega^{y} \omega^{z}\right)+\mathbf{V}_{\mathrm{d}, \mathrm{sh}, \mathrm{su}}^{\mathrm{y}}\left(\dot{\omega}^{z}-\omega^{x} \omega^{y}\right)-\mathbf{V}_{\mathrm{d}, \mathrm{sh}, \mathrm{su}}^{\theta}\left(\dot{\omega}^{x}+\omega^{y} \omega^{z}\right)-\mathbf{V}_{\mathrm{d}, \mathrm{sh}, \mathrm{su}}^{\psi}\left(\dot{\omega}^{z}-\omega^{x} \omega^{y}\right) \\
& -\mathbf{V}_{\mathrm{d}, \mathrm{sh}, \mathrm{su}}^{y \psi}\left(\Omega \omega^{x}+\omega^{x} \omega^{y}\right)+\mathbf{V}_{\mathrm{d}, \mathrm{sh}, \mathrm{su}}^{\mathrm{\theta}}\left(\Omega \omega^{z}+\omega^{y} \omega^{z}\right) \\
& \mathbf{F}_{\mathrm{d}, \mathrm{sh}, \mathrm{su}}^{\mathrm{c}_{2}}(t)=-\mathbf{V}_{\mathrm{d}, \mathrm{ch}, \mathrm{su}}^{\theta, c_{2}}\left(\dot{\omega}^{x}-2 \Omega \omega^{z}-\omega^{y} \omega^{z}\right)+\mathbf{V}_{\mathrm{d}, \mathrm{sh}, \mathrm{su}}^{\psi, c_{2}}\left(\dot{\omega}^{z}+2 \Omega \omega^{x}+\omega^{x} \omega^{y}\right) \\
& \mathbf{F}_{\mathrm{d}, \mathrm{sh}, \mathrm{su}}^{\mathrm{s}_{2}}(t)=\mathbf{V}_{\mathrm{d}, \mathrm{sh}, \mathrm{su}}^{\psi, \mathrm{s}_{2}}\left(\dot{\omega}^{x}-2 \Omega \omega^{z}-\omega^{y} \omega^{z}\right)+\mathbf{V}_{\mathrm{d}, \mathrm{sh}, \text { su }}^{\theta, \mathrm{s}_{2}}\left(\dot{\omega}^{z}+2 \Omega \omega^{x}+\omega^{x} \omega^{y}\right)
\end{aligned}
$$

where the load vectors $\mathbf{V}_{\mathrm{mu}}, \mathbf{V}_{\mathrm{d}, \mathrm{sh}, \mathrm{su}}$ and $\mathbf{V}_{\mathrm{mu}, \mathrm{su}}$ and their corresponding force vectors $\mathbf{F}_{\mathrm{mu}}, \mathbf{F}_{\mathrm{d}, \mathrm{sh}, \mathrm{su}}$ and $\mathbf{F}_{\mathrm{mu}, \mathrm{su}}$ are associated respectively with the mass unbalance, the inertia force due to support motions and that due to coupling between both phenomena. The superscripts " $\mathrm{c}_{1}$ " and " $\mathrm{s}_{1}$ " signify the components of the mass unbalance force expressed in terms of the time-varying trigonometric functions $\cos \Omega t$ and $\sin \Omega t$. The superscripts $u, w, \psi$ and $\theta$ denote the direction of the action force components associated with the rotor support motions.

For the sake of conciseness, the elementary matrices involved in $\mathbf{M}_{\mathrm{r}}(t), \mathbf{C}_{\mathrm{r}}(t), \mathbf{K}_{\mathrm{r}}(t)$ and the elementary vectors involved in $\mathbf{F}_{\mathrm{r}}(t)$ as well as the validation of the finite element calculations against the Rayleigh-Ritz formulation are not detailed here and can be seen in [35].

As already stated above, the equations of motion are non-linear. This is due to the hydrodynamic bearings (local components) even if they do not concern all the degrees of freedom of the rotor system. In the two following sections (i.e., Sections 5.3 and 5.4), the treatment of these equations is detailed.

\subsection{Non-linear dynamic analysis}

\subsubsection{Classical transient analysis}

For large hydrodynamic bearing forces and thereby large displacements of the rotor, the linear dynamic analysis is no longer valid. In this case, the non-linear analysis of a bearing is considered. Solving the complete non-linear dynamic problem requires the simultaneous solution of the equations of motion (i.e., Eq. (23)) of the rotor-bearing system and Eqs. (13)-(15) calculating the nonlinear bearing forces obtained by the Reynolds equation in the dynamic regime. As a consequence, the analysis uses a complex solving algorithm combining the Newton-Raphson incremental-iterative procedure with the Newmark time integration scheme. The semi-discrete equations of motion (Eq. (23)) applied at time $t_{i+1}\left(1 \leq i \leq n_{\mathrm{ts}}+1\right.$ where $n_{\mathrm{ts}}$ is the number of time steps) can yield the following residual form

$$
\mathbf{G}_{\mathrm{r}}\left(\boldsymbol{\delta}_{\mathrm{r}}^{i+1}\right)=\mathbf{M}_{\mathrm{r}}\left(t_{i+1}\right) \ddot{\boldsymbol{\delta}}_{\mathrm{r}}^{i+1}+\mathbf{C}_{\mathrm{r}}\left(t_{i+1}\right) \dot{\boldsymbol{\delta}}_{\mathrm{r}}^{i+1}+\mathbf{K}_{\mathrm{r}}\left(t_{i+1}\right) \boldsymbol{\delta}_{\mathrm{r}}^{i+1}-\mathbf{F}_{\mathrm{r}}\left(t_{i+1}\right)-\mathbf{F}_{\mathrm{be}}\left(\boldsymbol{\delta}_{\mathrm{r}}^{i+1}, \dot{\boldsymbol{\delta}}_{\mathrm{r}}^{i+1}\right)=\mathbf{0}
$$

The velocity and displacement vectors of the rotor nodes between $t_{i}$ and $t_{i+1}=t_{i}+\Delta t, \Delta t$ being the time step, are approximated by the following general formulations of the implicit Newmark scheme

$$
\dot{\boldsymbol{\delta}}_{\mathrm{r}}^{i+1}=\dot{\boldsymbol{\delta}}_{\mathrm{r}}^{i}+\Delta t\left(\xi_{1} \ddot{\mathbf{\delta}}_{\mathrm{r}}^{i+1}+\left(1-\xi_{1}\right) \ddot{\boldsymbol{\delta}}_{\mathrm{r}}^{i}\right) \quad ; \quad \boldsymbol{\delta}_{\mathrm{r}}^{i+1}=\boldsymbol{\delta}_{\mathrm{r}}^{i}+\Delta t \dot{\boldsymbol{\delta}}_{\mathrm{r}}^{i}+\frac{(\Delta t)^{2}}{2}\left(2 \xi_{\mathbf{2}} \ddot{\boldsymbol{\delta}}_{\mathrm{r}}^{i+1}+\left(1-2 \xi_{2}\right) \ddot{\boldsymbol{\delta}}_{\mathrm{r}}^{i}\right)
$$

where the parameters $\xi_{1}=1 / 2$ and $\xi_{2}=1 / 4$ define the constant average acceleration scheme and assume an unconditional numerical stability and second-order accuracy. Since Eq. (33) is non-linear, an iterative strategy is used to treat the non-linearity. Namely, the consistent linearization of Eq. (33) is applied by finding a first-order Taylor series of this equation and the Newton-Raphson iterative algorithm is taken into account as follows

$$
\mathbf{G}_{\mathrm{r}}\left({ }^{k+1} \boldsymbol{\delta}_{\mathrm{r}}^{i+1}\right) \simeq \mathbf{G}_{\mathrm{r}}\left({ }^{k} \boldsymbol{\delta}_{\mathrm{r}}^{i+1}\right)+\left.\frac{\mathrm{d} \mathbf{G}_{\mathrm{r}}}{\mathrm{d} \boldsymbol{\delta}_{\mathrm{r}}^{i+1}}\right|_{\left({ }^{k} \boldsymbol{\delta}_{r}^{i+1}\right)} \Delta \boldsymbol{\delta}_{\mathrm{r}}^{\mathrm{NR}}=\mathbf{0} \Rightarrow \mathbf{J}_{\mathrm{r}}\left({ }^{k} \boldsymbol{\delta}_{\mathrm{r}}^{i+1}\right) \Delta \boldsymbol{\delta}_{\mathrm{r}}^{\mathrm{NR}}=-\mathbf{G}_{\mathrm{r}}\left({ }^{k} \boldsymbol{\delta}_{\mathrm{r}}^{i+1}\right)
$$

where $\Delta \boldsymbol{\delta}_{\mathrm{r}}^{\mathrm{NR}}={ }^{k+1} \boldsymbol{\delta}_{\mathrm{r}}^{i+1}-{ }^{k} \boldsymbol{\delta}_{\mathrm{r}}^{i+1}$ is the iterative increment of the displacement vector and $k$ is the Newton-Raphson iteration. The Jacobian matrix $\mathbf{J}_{\mathrm{r}}$ is a function of the displacement vector $\boldsymbol{\delta}_{\mathrm{r}}^{i+1}$ at iteration $k$ and is given by

$$
\mathbf{J}_{\mathrm{r}}\left({ }^{k} \boldsymbol{\delta}_{\mathrm{r}}^{i+1}\right)=\left.\frac{\mathrm{d} \mathbf{G}_{\mathrm{r}}}{\mathrm{d} \boldsymbol{\delta}_{\mathrm{r}}^{i+1}}\right|_{\left({ }^{k} \boldsymbol{\delta}_{r}^{i+1}\right)}=\mathbf{M}_{\mathrm{r}}\left(t_{i+1}\right) \frac{\mathrm{d} \ddot{\boldsymbol{\delta}}_{\mathrm{r}}^{i+1}}{\mathrm{~d} \boldsymbol{\delta}_{\mathrm{r}}^{i+1}}+\mathbf{C}_{\mathrm{r}}\left(t_{i+1}\right) \frac{\mathrm{d} \dot{\boldsymbol{\delta}}_{\mathrm{r}}^{i+1}}{\mathrm{~d} \boldsymbol{\delta}_{\mathrm{r}}^{i+1}}+\mathbf{K}_{\mathrm{r}}\left(t_{i+1}\right)-\left.\frac{\mathrm{d} \mathbf{F}_{b e}}{\mathrm{~d} \boldsymbol{\delta}_{\mathrm{r}}^{i+1}}\right|_{\left({ }^{(} \boldsymbol{\delta}_{r}^{i+1}, \dot{\boldsymbol{\delta}}_{\mathrm{r}}^{i+1}\right)} \quad \text { with } \frac{\mathrm{d} \ddot{\boldsymbol{\delta}}_{\mathrm{r}}^{i+1}}{\mathrm{~d} \boldsymbol{\delta}_{\mathrm{r}}^{i+1}}=\frac{1}{\xi_{2}(\Delta t)^{2}} \mathbf{I} \quad ; \quad \frac{\mathrm{d} \dot{\boldsymbol{\delta}}_{\mathrm{r}}^{i+1}}{\mathrm{~d} \boldsymbol{\delta}_{\mathrm{r}}^{i+1}}=\frac{\xi_{1}}{\xi_{2} \Delta t} \mathbf{I}
$$


where $\mathbf{I}$ is the identity matrix. The derivatives of the acceleration and velocity vectors $\ddot{\boldsymbol{\delta}}_{\mathrm{r}}^{i+1}, \dot{\boldsymbol{\delta}}_{\mathrm{r}}^{i+1}$ with respect to the displacement vector $\boldsymbol{\delta}_{\mathrm{r}}^{i+1}$ are deduced from the approximations of the Newmark scheme (i.e., Eq.(34)). The derivative of the non-linear hydrodynamic forces in the Jacobian matrix of Eq. (36) depends on both the displacement and velocity vectors $\left({ }^{k} \boldsymbol{\delta}_{\mathrm{r}}^{i+1},{ }^{k} \dot{\boldsymbol{\delta}}_{\mathrm{r}}^{i+1}\right)$, is updated at each iteration $k$ and is expressed in what follows

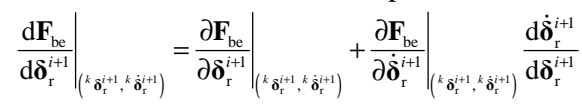

The algorithm of the Newton-Raphson incremental-iterative procedure combined with the Newmark time integration scheme is presented in Fig. 3. The initial dynamic displacement vector is set to the static equilibrium position $\boldsymbol{\delta}_{\mathrm{r}}^{0}$ (or to other user-defined vectors) and the initial velocity vector is set to zero. When the residual vector $\mathbf{G}_{\mathrm{r}}\left({ }^{k} \boldsymbol{\delta}_{\mathrm{r}}^{i+1}\right)$ is calculated by Eq. (33), a new displacement increment $\Delta \boldsymbol{\delta}_{\mathrm{r}}^{\mathrm{NR}}$ is produced by Eq. (35) and thus the displacement, velocity and acceleration vectors are updated as shown in Fig. 3. If the ratio $\left\|\mathbf{G}_{\mathrm{r}}\left({ }^{k+1} \boldsymbol{\delta}_{\mathrm{r}}^{i+1}\right)\right\| /\left\|\mathbf{F}_{\mathrm{r}}\left(t_{i+1}\right)\right\|$ is less than a convergence criterion $\varepsilon_{\mathrm{nl}}^{\mathrm{NR}}$, then a converged solution is reached and the algorithm shifts to the next time step of the Newmark scheme. Otherwise the non-linear bearing forces are re-evaluated and a new NewtonRaphson iteration begins.

In order to reach the steady-state dynamic response in the periodic regime, the Newmark integration scheme must be used over a large number of time periods in the case of a harmonic external excitation of the system. This results in a huge computational time when the indicated scheme is repeated for a large number of fundamental excitation frequencies, i.e., each fundamental frequency corresponds to a large number of time periods. Computational time can be saved by computing directly the periodic solution by means of the shooting algorithm which is discussed in the next section.

\subsubsection{Direct computation of the periodic solution (shooting method)}

The following two-point boundary-value problem defined by the periodicity condition is considered, then

$$
\mathbf{H}_{\mathrm{r}}\left(\mathbf{X}_{\mathrm{r}}(0), t=\tau_{\mathrm{r}}\right)=\mathbf{X}_{\mathrm{r}}\left(\mathbf{X}_{\mathrm{r}}(0), t=\tau_{\mathrm{r}}\right)-\mathbf{X}_{\mathrm{r}}(0)=\mathbf{0} \quad \text { with } \quad \mathbf{X}_{\mathrm{r}}=\left[\boldsymbol{\delta}_{\mathrm{r}}, \dot{\boldsymbol{\delta}}_{\mathrm{r}}\right]^{\mathrm{T}}
$$

where $\tau_{\mathrm{r}}$ is the minimal period of the rotor response. The dependence of the system on the fundamental period $\tau_{\mathrm{ex}}$ of the harmonic exciting forces is assumed, i.e., $\tau_{\mathrm{r}}=j \tau_{\mathrm{ex}}$ (with $j=1,2,3 \ldots$ ). Integer multiples of the fundamental period are used to compute subharmonic responses occurring, for instance, after a period-doubling bifurcation. In practice, in order to find the minimal period $\tau_{\mathrm{r}}$ of the response, the shooting algorithm is first run with $\tau_{\mathrm{r}}=\tau_{\mathrm{ex}}$ (harmonic response) and if there is no convergence, it is then run with $\tau_{\mathrm{r}}=2 \tau_{\mathrm{ex}}, \tau_{\mathrm{r}}=3 \tau_{e x}, \ldots$ consecutively (sub-harmonic response). The state-space vector $\mathbf{X}_{\mathrm{r}}$ in Eq. (38) represents the solution of Eq. (23) at the end of one period $\tau_{\mathrm{r}}$ for an approximated initial solution $\mathbf{X}_{\mathrm{r}}(0)$. This vector is obtained with the non-linear Newmark time integration scheme over one period $\tau_{\mathrm{r}}$ (see Section 5.3.1 and Fig. 3). An appropriate linearization of Eq. (38) is applied by building a first-order Taylor series expansion of this equation and a Newton-Raphson iterative correction is performed, then

$$
\mathbf{H}_{\mathrm{r}}\left({ }^{k+1} \mathbf{X}_{\mathrm{r}}(0), \tau_{\mathrm{r}}\right) \simeq \mathbf{H}_{\mathrm{r}}\left({ }^{k} \mathbf{X}_{\mathrm{r}}(0), \tau_{\mathrm{r}}\right)+\left.\frac{\partial \mathbf{H}_{\mathrm{r}}}{\partial \mathbf{X}_{\mathrm{r}}(0)}\right|_{\left({ }^{k} \mathbf{X}_{\mathrm{r}}(0), \tau_{\mathrm{r}}\right)} \Delta \mathbf{X}_{\mathrm{r}}^{\mathrm{SH}}=\mathbf{0} \Rightarrow \mathbf{J}_{\mathrm{r}}^{\mathrm{SH}}\left({ }^{k} \mathbf{X}_{\mathrm{r}}(0), \tau_{\mathrm{r}}\right) \Delta \mathbf{X}_{\mathrm{r}}^{\mathrm{SH}}=-\mathbf{H}_{\mathrm{r}}\left({ }^{k} \mathbf{X}_{\mathrm{r}}(0), \tau_{\mathrm{r}}\right)
$$

where $\Delta \mathbf{X}_{\mathrm{r}}^{\mathrm{SH}}={ }^{k+1} \mathbf{X}_{\mathrm{r}}(0)-{ }^{k} \mathbf{X}_{\mathrm{r}}(0)$ is the iterative increment of the state-space vector and $k$ is the Newton-Raphson iteration. The Jacobian matrix $\mathbf{J}_{\mathrm{r}}^{\mathrm{SH}}$ is a function of the state-space vector $\mathbf{X}_{\mathrm{r}}(0)$ at iteration $k$ and is given by

$$
\mathbf{J}_{\mathrm{r}}^{\mathrm{SH}}\left({ }^{k} \mathbf{X}_{\mathrm{r}}(0), \tau_{\mathrm{r}}\right)=\left.\frac{\partial \mathbf{H}_{\mathrm{r}}}{\partial \mathbf{X}_{\mathrm{r}}(0)}\right|_{\left({ }^{k} \mathbf{X}_{\mathrm{r}}(0), \tau_{\mathrm{r}}\right)}=\left.\frac{\partial \mathbf{X}_{\mathrm{r}}}{\partial \mathbf{X}_{\mathrm{r}}(0)}\right|_{\left({ }^{k} \mathbf{X}_{\mathrm{r}}(0), \tau_{\mathrm{r}}\right)}-\mathbf{I}
$$

The shooting method algorithm solving simultaneously Eqs. (23) and (38) is presented in Fig. 4. The approximated initial solution is set to a user-defined state-space vector ${ }^{1} \mathbf{X}_{\mathrm{r}}(0)$. When the residual vector $\mathbf{H}_{\mathrm{r}}\left({ }^{k} \mathbf{X}_{\mathrm{r}}(0), \tau_{\mathrm{r}}\right)$ is calculated by Eq. (38), a new increment $\Delta \mathbf{X}_{\mathrm{r}}^{\mathrm{SH}}$ of the state-space vector is produced by Eq. (39) and thus the approximated initial solution ${ }^{k+1} \mathbf{X}_{\mathrm{r}}(0)$ is updated as shown in Fig. 4. If the ratio $\left\|\mathbf{H}_{\mathrm{r}}\left({ }^{k} \mathbf{X}_{\mathrm{r}}(0), \tau_{\mathrm{r}}\right)\right\| /\left\|\mathbf{X}_{\mathrm{r}}\left({ }^{k} \mathbf{X}_{\mathrm{r}}(0), t=\tau_{\mathrm{r}}\right)\right\|$ is less than a convergence criterion $\varepsilon^{\mathrm{sH}}$, then a converged solution is reached and the algorithm is terminated. Otherwise the state-space vector $\mathbf{X}_{\mathrm{r}}\left({ }^{k} \mathbf{X}_{\mathrm{r}}(0), t=\tau_{\mathrm{r}}\right)$ is re-calculated and a new Newton-Raphson iteration begins.

\subsection{Linear dynamic analysis}

\subsubsection{Transient analysis}

The solution technique can be significantly simplified and the computational time can be reduced if the assumption of a linearized 8 -coefficient hydrodynamic bearing model is considered to be valid, i.e., the dynamic displacement vector of the rotor remains small in the vicinity of the static position $\boldsymbol{\delta}_{\mathrm{r}}^{0}$ and a first-order Taylor expansion of the dynamic fluid forces $\mathbf{F}_{\mathrm{be}}\left(\boldsymbol{\delta}_{\mathrm{r}}, \dot{\boldsymbol{\delta}}_{\mathrm{r}}\right)$ is built by

$$
\mathbf{F}_{\mathrm{be}}\left(\boldsymbol{\delta}_{\mathrm{r}}, \dot{\boldsymbol{\delta}}_{\mathrm{r}}\right)=\mathbf{F}_{\mathrm{be}}\left(\boldsymbol{\delta}_{\mathrm{r}}^{0}, \mathbf{0}\right)-\mathbf{C}_{\mathrm{be}} \Delta \dot{\boldsymbol{\delta}}_{\mathrm{r}}-\mathbf{K}_{\mathrm{be}} \Delta \boldsymbol{\delta}_{\mathrm{r}}
$$


with $\Delta \boldsymbol{\delta}_{\mathrm{r}}=\boldsymbol{\delta}_{\mathrm{r}}-\boldsymbol{\delta}_{\mathrm{r}}^{0}$ and $\Delta \dot{\boldsymbol{\delta}}_{\mathrm{r}}=\dot{\boldsymbol{\delta}}_{\mathrm{r}}$. The hydrodynamic force vector $\mathbf{F}_{\mathrm{be}}\left(\boldsymbol{\delta}_{\mathrm{r}}^{0}, \mathbf{0}\right)$ depends on the static solution vector $\boldsymbol{\delta}_{\mathrm{r}}^{0}$ caused by the static equilibrium between the rotor weight vector $\mathbf{F}_{\mathrm{d}, \mathrm{s}}^{W_{r}}$ and the hydrodynamic bearing reaction (i.e., $\mathbf{F}_{\mathrm{be}}\left(\boldsymbol{\delta}_{\mathrm{r}}^{0}, \mathbf{0}\right)$ is opposite and equal to $\mathbf{F}_{\mathrm{d}, \mathrm{sh}}^{W_{\mathrm{s}}}$ ). The global damping and stiffness matrices $\mathbf{C}_{\mathrm{be}}, \mathbf{K}_{\mathrm{be}}$ deduced from the bearing linearization (see Section 4.4) are generated by the assembly of the elementary matrices. These matrices are kept constant all along the dynamic simulation. Substituting Eq. (41) into Eq. (23), the equations of motion of the on-board rotor-bearing system become linear as follows

$$
\mathbf{M}_{\mathrm{r}}(t) \ddot{\boldsymbol{\delta}}_{\mathrm{r}}+\left(\mathbf{C}_{\mathrm{r}}(t)+\mathbf{C}_{\mathrm{be}}\right) \dot{\boldsymbol{\delta}}_{\mathrm{r}}+\left(\mathbf{K}_{\mathrm{r}}(t)+\mathbf{K}_{\mathrm{be}}\right) \boldsymbol{\delta}_{\mathrm{r}}=\mathbf{F}_{\mathrm{r}}(t)+\mathbf{F}_{\mathrm{be}}\left(\boldsymbol{\delta}_{\mathrm{r}}^{0}, \mathbf{0}\right)+\mathbf{K}_{\mathrm{be}} \boldsymbol{\delta}_{\mathrm{r}}^{0}
$$

The transient dynamic motion of the rotor nodes is obtained by solving Eq. (42) by means of the implicit Newmark time-step integration algorithm based on the average acceleration. The displacement vector $\boldsymbol{\delta}_{\mathrm{r}}^{0}$ of the static equilibrium position $C_{\mathrm{be}}^{0}$ is used to initialize the transient dynamic problem. The final integration time is selected such that the transient effects have disappeared and the steady-state regime has been reached. Lastly, it is necessary to establish precisely the validity range of the linear bearing model by comparing its results with those of the non-linear bearing model.

\subsubsection{Stability analysis}

The motion stability of the linear rotor-bearing system is investigated by applying the Floquet theory for one period $\tau_{\mathrm{pe}}$ of the periodic parametric excitation, see Dufour and Berlioz [6] and Dakel et al. [35]. In other words, by calculating the so-called "Floquet transition matrix" (or monodromy matrix) during one period $\tau_{\mathrm{pe}}$ of the parametric excitation and by finding its eigenvalues, the zones of instability are identified, i.e., if the modulus of at least one of these eigenvalues is greater than 1, the system is unstable. As reported in [37], the Floquet transition matrix can be very efficiently computed using the linear version of the Newmark algorithm.

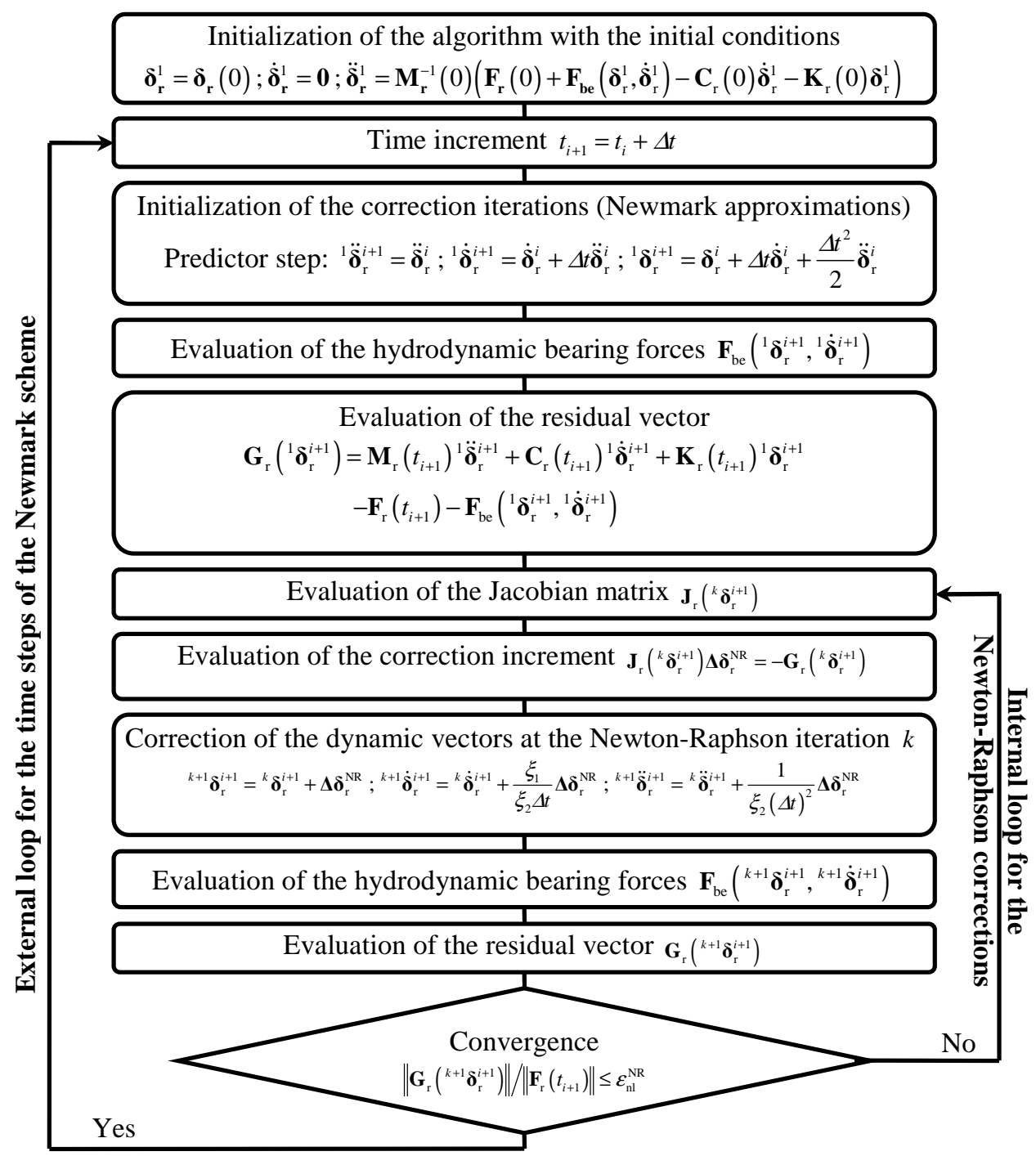

Fig. 3. Algorithm used for the non-linear transient analysis of the on-board rotor-hydrodynamic bearing system. 


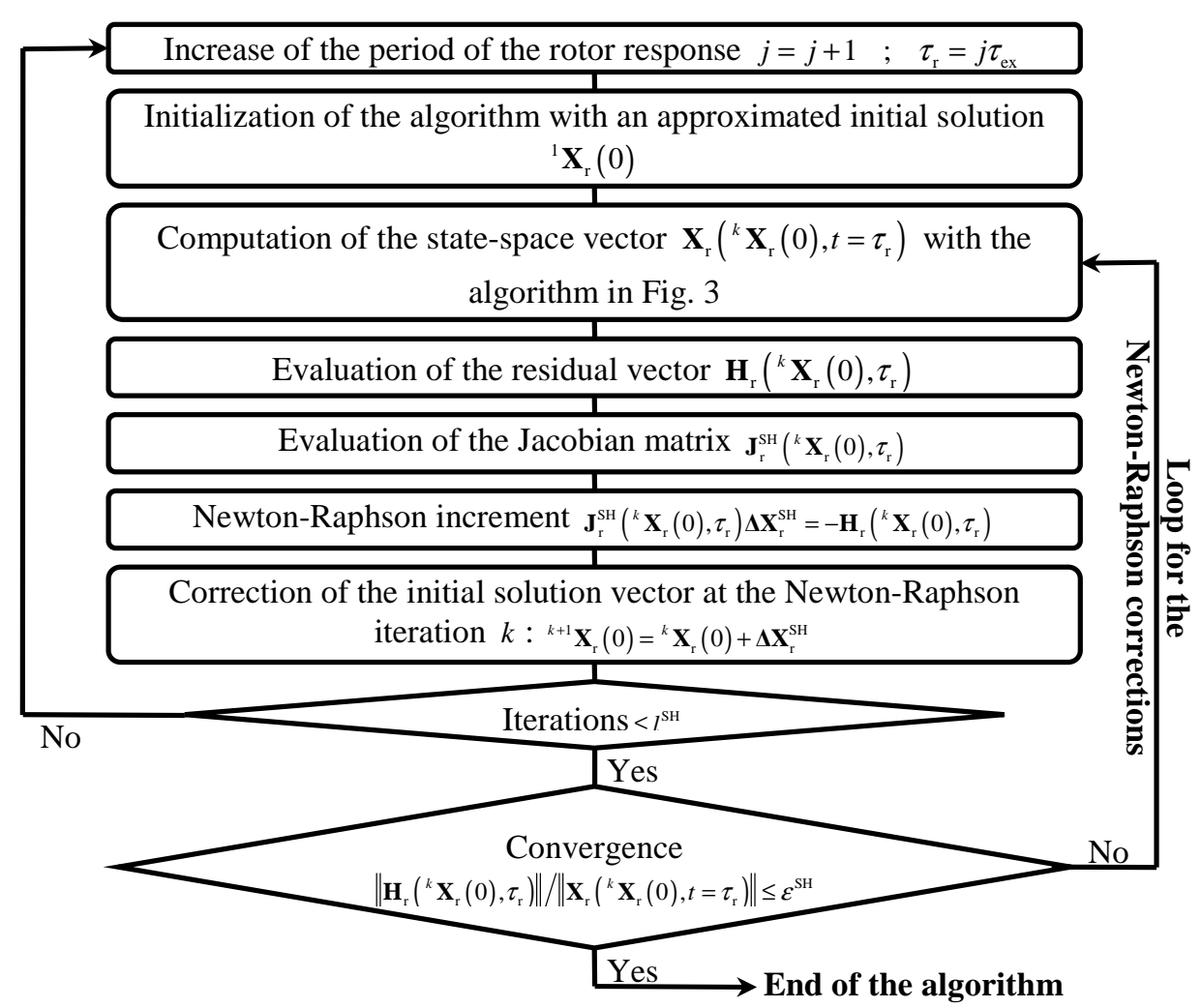

Fig. 4. Shooting method algorithm used for the non-linear dynamic analysis of the on-board rotor.

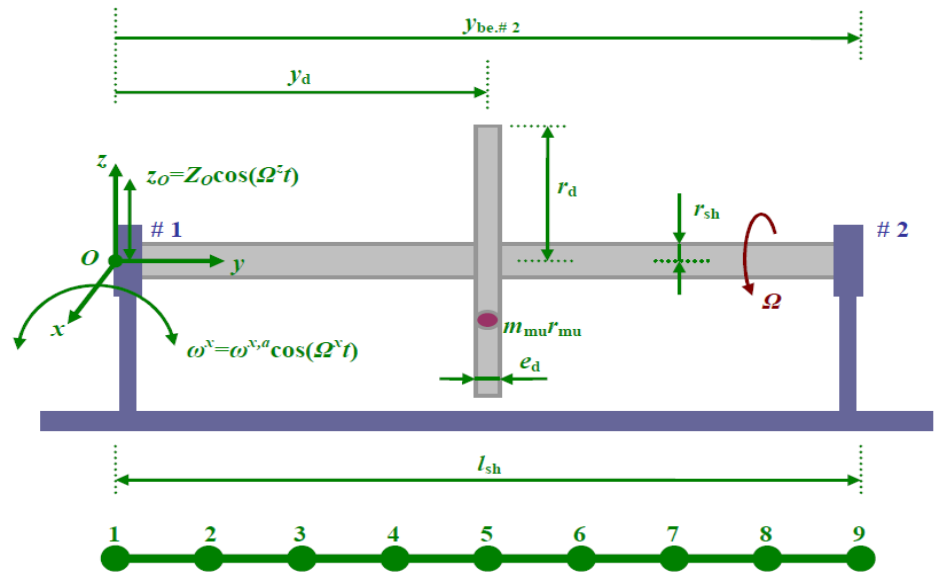

Fig. 5. On-board rotor-hydrodynamic bearing system configuration.

Table 1. Main characteristics of the on-board rotor.

Density of both disk and shaft material

Radius, thickness and location of the disk

Radius and length of the shaft

$\rho_{\mathrm{d}}=\rho_{\mathrm{sh}}=7800 \mathrm{~kg} / \mathrm{m}^{3}$

$r_{\mathrm{d}}=0.15 \mathrm{~m}, e_{\mathrm{d}}=0.03 \mathrm{~m}, y_{\mathrm{d}}=0.2 \mathrm{~m}$

Young's modulus and Poisson's ratio of the shaft $\quad E_{\mathrm{sh}}=2 \times 10^{11} \mathrm{~N} / \mathrm{m}^{2}, v_{\mathrm{sh}}=0.3$

Shear correction factor of the shaft $\quad \kappa_{\mathrm{sh}}{ }^{x}=\kappa_{\mathrm{sh}}{ }^{z}=0.8864$ [38]

Mass unbalance phase

$\eta_{\mathrm{mu}}=0^{\circ}$

Table 2. Main characteristics of the hydrodynamic short bearings.

Radius and length of the bearings

Locations of the bearings

Radial clearance of the bearings $r_{\mathrm{be}}=0.04 \mathrm{~m}, l_{\mathrm{be}}=0.01 \mathrm{~m}$

Oil film dynamic viscosity

$y_{\text {be.\# } 1}=0 \mathrm{~m}, y_{\text {be.\# } 2}=0.4 \mathrm{~m}$

$c_{\text {be }}=2 \times 10^{-4} \mathrm{~m}$

$\mu_{\mathrm{be}}=288 \times 10^{-4} \mathrm{~Pa} \mathrm{~s}$ 


\section{Numerical simulations and result discussion}

\subsection{Description of the investigated on-board rotor-bearing system}

The symmetric on-board rotor-hydrodynamic short bearing system is presented in Fig. 5. The origin $O$ of the reference frame $R$ coincides with the shaft end-point positioned at the center of the bearing \# 1 . The symmetric shaft is modeled with eight identical two-node Timoshenko beam finite elements, i.e., the total number of degrees of freedom is $n_{\mathrm{g}}=36$. The symmetric disk is located at node 5 and the mass unbalance is placed on it. The hydrodynamic short bearings \# 1 and \# 2 are located at nodes 1 and 9 respectively, i.e., the corresponding localized non-linear degrees of freedom are 1-4 and 33-36. The material characteristics and geometric properties of the rotor and the bearings are listed in Tables 1 and 2.

The investigated rotor is subject to rotating mass unbalance excitation combined with sinusoidal rotational or translational motions of the support: the rotation around the $O x$ axis is given by the angular velocity $\omega^{x}=\omega^{x, a} \cos \Omega^{x} t$ in $\mathrm{rad} / \mathrm{s}$ (i.e., the corresponding angular displacement is $\beta=\omega^{x, a} / \Omega^{x} \sin \Omega^{x} t$ in $\mathrm{rad}$ ), while the translation along the $O z$ axis is expressed as $z_{o}=Z_{O} \cos \Omega^{z} t$ in $\mathrm{m}$. This rotor runs at a constant speed of rotation $\Omega=1200 \mathrm{rpm}$ ( $=20 \mathrm{~Hz}=$ mass unbalance frequency) and its first four natural frequencies for the considered speed of rotation and a fixed support in the case of linearized bearings are $33.6 \mathrm{~Hz}$, $52.7 \mathrm{~Hz}, 524.8 \mathrm{~Hz}$ and $1573 \mathrm{~Hz}$. The relative coordinates of the static equilibrium position $C_{\mathrm{be}}^{0}$ of the shaft geometric center in the fluid film bearings are given by $\boldsymbol{\delta}_{\mathrm{be}}^{0} / c_{\mathrm{be}}=[-0.29,-0.88]_{R}^{\mathrm{T}}$ or equivalently by $e_{\mathrm{be}}^{0} / c_{\mathrm{be}}=0.93$. The damping and stiffness matrices of the linearized 8-coefficient bearing model obtained with Eq. (18) and data from Table 2 are expressed as follows

$$
\mathbf{c}_{\mathrm{be}}=\left[\begin{array}{ll}
3.50 \times 10^{3} & 1.08 \times 10^{4} \\
1.08 \times 10^{4} & 7.57 \times 10^{4}
\end{array}\right] \mathrm{N} \mathrm{s} / \mathrm{m} \quad ; \quad \mathbf{k}_{\mathrm{be}}=\left[\begin{array}{ll}
1.30 \times 10^{6} & 1.32 \times 10^{6} \\
6.30 \times 10^{6} & 1.94 \times 10^{7}
\end{array}\right] \mathrm{N} / \mathrm{m}
$$

The equations of motion of the on-board rotor (see Eqs. (23)-(32)) show that the support rotations have a parametric influence on these equations, while the support translations have only an influence on the external force vector. Due to the complexity of the rotor motion, the overall dynamic analysis of the on-board rotor is performed by means of stability charts, rotor orbits, time history responses, fast Fourier transforms (FFTs), bifurcation diagrams and Poincaré maps.

\subsection{Role of the hydrodynamic bearing models in the stability analysis}

\subsubsection{Linearized model}

In this section, the linearized equations of motion are considered (see Eq. (42)) and the dynamic stability of the on-board rotor is examined through the Floquet theory. The instability comes from the parametric excitation due to the sinusoidal rotation of the rotor support, see Eqs. (24)-(26). For a certain speed of rotation $\Omega$ of the rotor, two factors affect the parametric excitations generated in the symmetric rotor: the amplitude $\omega^{r, a}$ of the support rotation and its frequency $\Omega^{x}$. Therefore the stability analysis covers these two parameters and uses $\tau_{\mathrm{pe}}=\pi / \Omega^{x}$ as the period of the parametric excitation.

Fig. 6(a) and (b) compare the stability charts investigated in the presence of the rigid short bearings (for which the first four natural frequencies of the rotor at $\Omega=1200 \mathrm{rpm}$ are $552.8 \mathrm{~Hz}, 553.1 \mathrm{~Hz}, 1608 \mathrm{~Hz}$ and $1642.7 \mathrm{~Hz}$ ) and the linearized hydrodynamic short ones respectively. The frequency of the support rotation is contained in the range of interest $\Omega^{x} \in[0 ; 2000 \mathrm{~Hz}]$ and the calculations are performed with $\Delta \Omega^{x}=1 \mathrm{~Hz}$ and $\Delta\left(\omega^{x, a} / \Omega^{x}\right)=0.05 \mathrm{rad}$. It is noted that the size of the instability zones increases for increasing values of $\omega^{x, a} / \Omega^{x}$.

For example, in the case where $\omega^{x, a} / \Omega^{x}=0.5 \mathrm{rad}$, the on-board rotor mounted on the rigid bearings can be unstable for $\Omega^{x} \in[508 ; 536 \mathrm{~Hz}]$ and $\Omega^{x} \in[1507 ; 2000 \mathrm{~Hz}]$ (see Fig. 6(a)), while the rotor mounted on the flexible bearings can be unstable for $\Omega^{x} \in[370 ; 2000 \mathrm{~Hz}]$ (see Fig. 6(b)). Namely, the instability region due to the linearized hydrodynamic bearings is greater than that due to the rigid ones because of the presence of the damping in the hydrodynamic bearings.

(a)

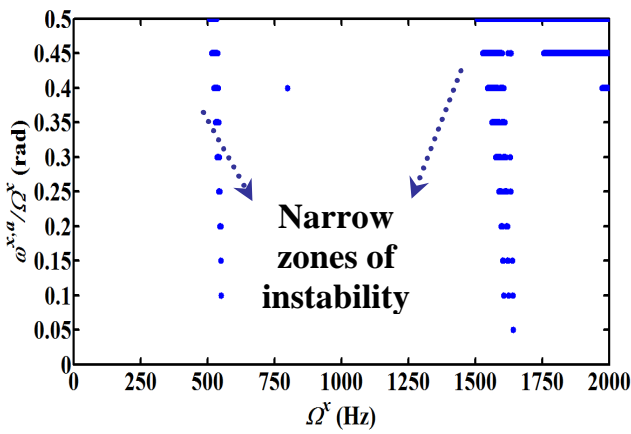

(b)

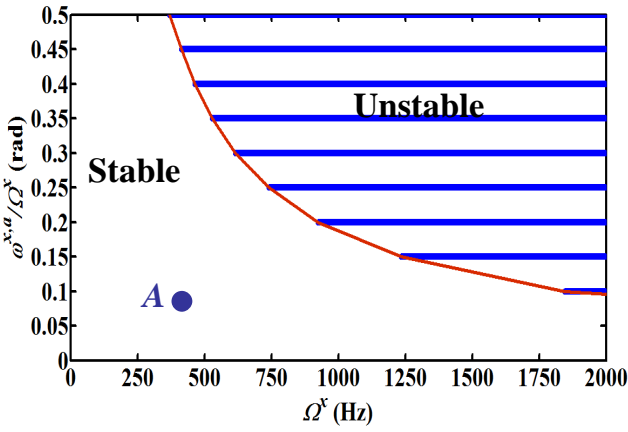

Fig. 6. Stability charts of the rotor running at $\Omega=1200 \mathrm{rpm}$, subject to sinusoidal rotations of the support and mounted on: rigid bearings, or (b) hydrodynamic bearings. 
(a)

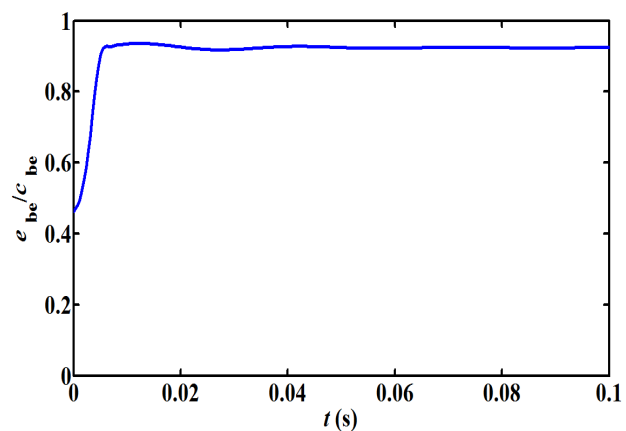

(b)

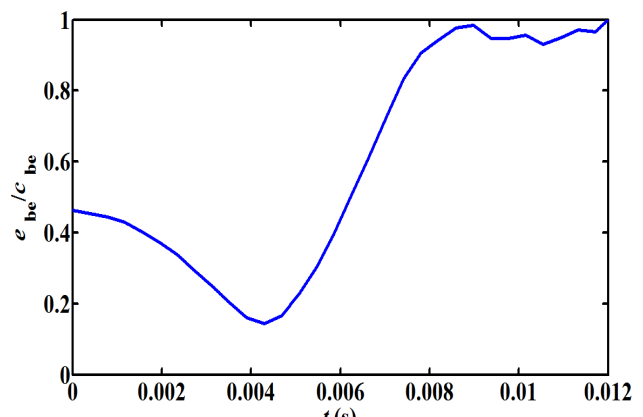

$t(\mathbf{s})$

Fig. 7. Relative non-linear time history responses of the rotor at the bearing \# 2 corresponding to the point $A$ in Fig. 6 with two different initial conditions.

\subsubsection{Non-linear model}

According to the stability analysis in the case of linearized 8-coefficient hydrodynamic bearings, any operating point below the transition curve between the stable and unstable zones is considered to be stable. Namely, the transient dynamic analysis of the linearized equations of motion of the rotor (see Eq. (42)) with no external forces except the rotor weight yields the static equilibrium position $C_{\mathrm{be}}^{0}$ of the shaft center in the bearings for any initial conditions. Unlike the previous case, due to the non-linear bearings, the chosen initial conditions for the transient analysis of the non-linear equations of motion (see Eq. (23)) with only the rotor weight as external forces can lead to an unstable response instead of the static position. This phenomenon is a form of instability occurring in hydrodynamic bearings and called "self-excited vibrations" or "non-loaded bearing vibrations", see Khonsari and Chang [12].

Fig. 7 presents the relative dynamic radial displacement of the rotor at the bearing \# 2 (i.e., node 9) in the presence of a non-linear bearing model and a sinusoidal rotation of the support of frequency $\Omega^{x}=500 \mathrm{~Hz}$. The dynamic displacement in the time domain corresponding to the point $A$ (Fig. 6(b)) with the initial conditions $\boldsymbol{\delta}_{\text {be }}^{0} / 2 c_{\text {be }}$ (see Fig. 7(a)) settles into a stable point corresponding to the static position $C_{\mathrm{be}}^{0}$ but becomes unstable with the initial conditions $-\boldsymbol{\delta}_{\mathrm{be}}^{0} / 2 c_{\mathrm{be}}$ (see Fig. $7(\mathrm{~b})$ ). In the second case of initial conditions, the non-loaded bearing vibrations appear. Moreover, a contact between the rotor and the bearing occurs at the instant $t=0.012 \mathrm{~s}$ and the transient dynamic computation is stopped.

\subsection{Comparison of linear and non-linear bearing models}

\subsubsection{Influence of the mass unbalance}

All the orbits presented in this paper are made dimensionless with respect to the bearing clearance $c_{\mathrm{be}}$ and are thus plotted within a unit circle.

In this section, the dynamic simulation is performed to present a comparison between rotor orbits obtained with linearized 8coefficient and non-linear hydrodynamic bearing models due to three different values of mass unbalance. Since the bearings are identical and the disk is positioned at the middle of the shaft (i.e., node 5), the orbits at both bearings (i.e., nodes 1 and 9) are identical in the case of a fixed support. These orbits illustrated in Fig. 8 show the transient motion of the shaft geometric center within the bearings from the static position $C_{\mathrm{be}}^{0}$ to the steady-state harmonic solution which has a period $\tau_{\mathrm{r}}$ equal to the inverse of the mass unbalance frequency $\Omega$. The periodic non-linear orbits plotted in Fig. 8 are also obtained with the shooting method. As expected, they perfectly coincide with the steady-state periodic motion obtained by the transient time integration and thus validate the shooting implementation.

For a small mass unbalance ( $m_{\mathrm{mu}} r_{\mathrm{mu}}=750 \mathrm{~g} \mathrm{~mm}$ ), the linear and non-linear orbits of the rotor exhibit very similar evolutions (see Fig. 8(a)). According to Fig. 8(b), the chosen mass unbalance $\left(m_{\mathrm{mu}} r_{\mathrm{mu}}=1500 \mathrm{~g} \mathrm{~mm}\right)$ corresponds to the limit of validity of the linearized bearing model and is thus used in the next sections. For a very large mass unbalance $\left(m_{\mathrm{mu}} r_{\mathrm{mu}}=7500 \mathrm{~g} \mathrm{~mm}\right)$, the dynamic displacement of the rotor becomes much greater and no longer remains in the vicinity of the static position and a high difference between the linear and non-linear orbits shown in Fig. 8(c) is noted. As a consequence, the assumption of constant damping and stiffness coefficients of the bearings is no longer valid and the linearized 8-coefficient bearing model yields inaccurate results.

\subsubsection{Influence of the support motion frequency}

Figs. 9 and 10 display a comparison between linear orbits of the rotor and non-linear ones (obtained by the transient Newmark integration and the shooting algorithm) at the bearing \#2 (node 9) in the presence of the mass unbalance excitation $\left(m_{\mathrm{mu}} r_{\mathrm{mu}}=1500 \mathrm{~g} \mathrm{~mm}\right)$ and the sinusoidal rotational or translational motions of the rotor support for the initial conditions $\boldsymbol{\delta}_{\mathrm{be}}^{0} / c_{\mathrm{be}}$. In

Fig. 9, the amplitude of the support rotation is kept constant $\left(\omega^{x, a}=1 \times 10^{-2} \mathrm{rad} / \mathrm{s}\right)$, while its frequency varies ( $\Omega^{x}=80 \mathrm{~Hz}, 120 \mathrm{~Hz}$ and $200 \mathrm{~Hz}$ ), i.e., the corresponding angular displacement amplitudes of the support are $\omega^{x, a} / \Omega^{x}=2 \times 10^{-5} \mathrm{rad}, 1.33 \times 10^{-5} \mathrm{rad}$ and $7.96 \times 10^{-6} \mathrm{rad}$ respectively. In Fig. 10, the amplitude of the support translation is kept constant $\left(Z_{O}=1 \times 10^{-5} \mathrm{~m}\right)$, while its frequency varies $\left(\Omega^{z}=80 \mathrm{~Hz}, 120 \mathrm{~Hz}\right.$ and $\left.200 \mathrm{~Hz}\right)$. 


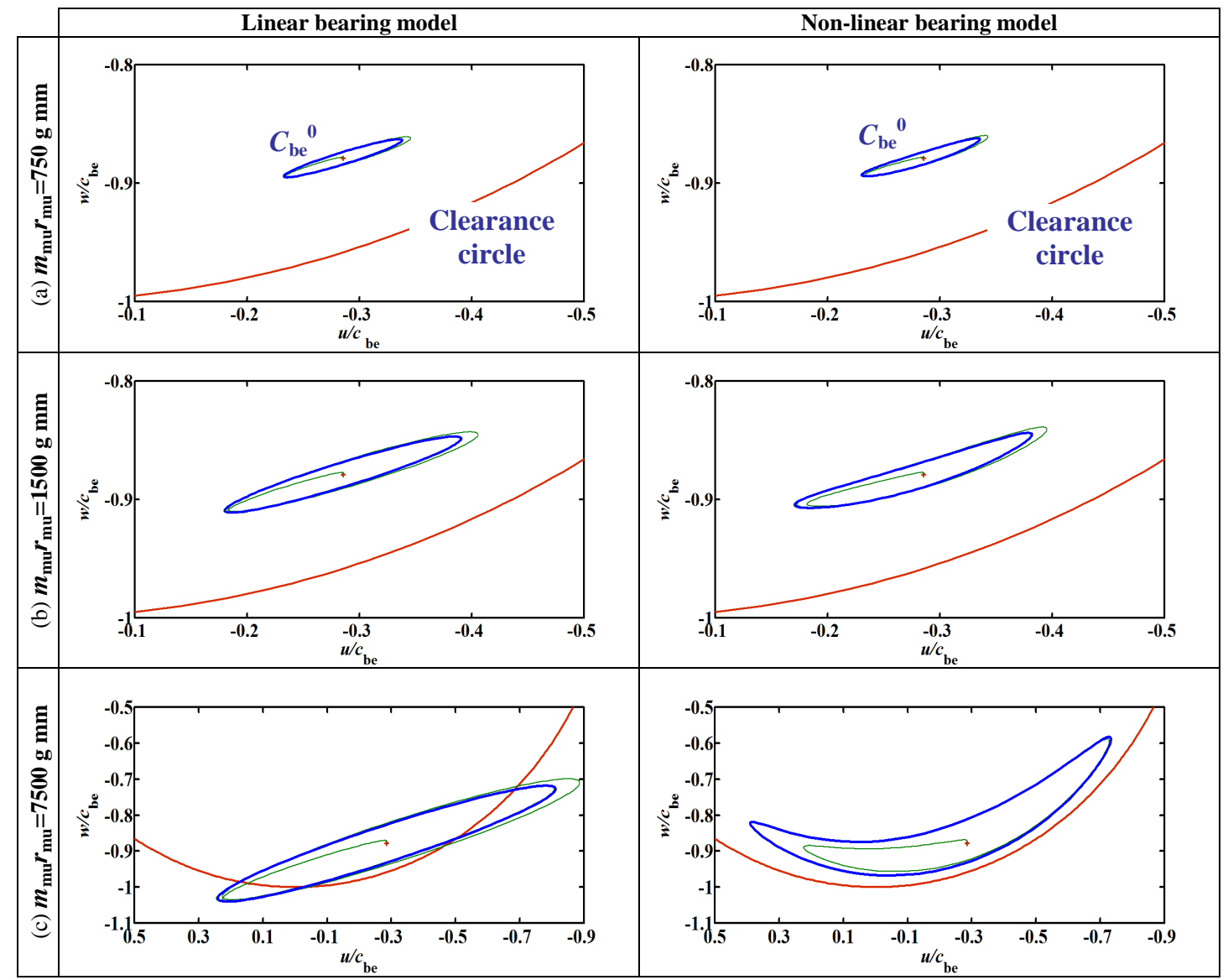

Fig. 8. Comparison of linear and non-linear orbits of the rotor at both bearings for three different values of mass unbalance: $m_{\mathrm{mu}} r_{\mathrm{mu}}=(\mathrm{a}) 750 \mathrm{~g} \mathrm{~mm}$,

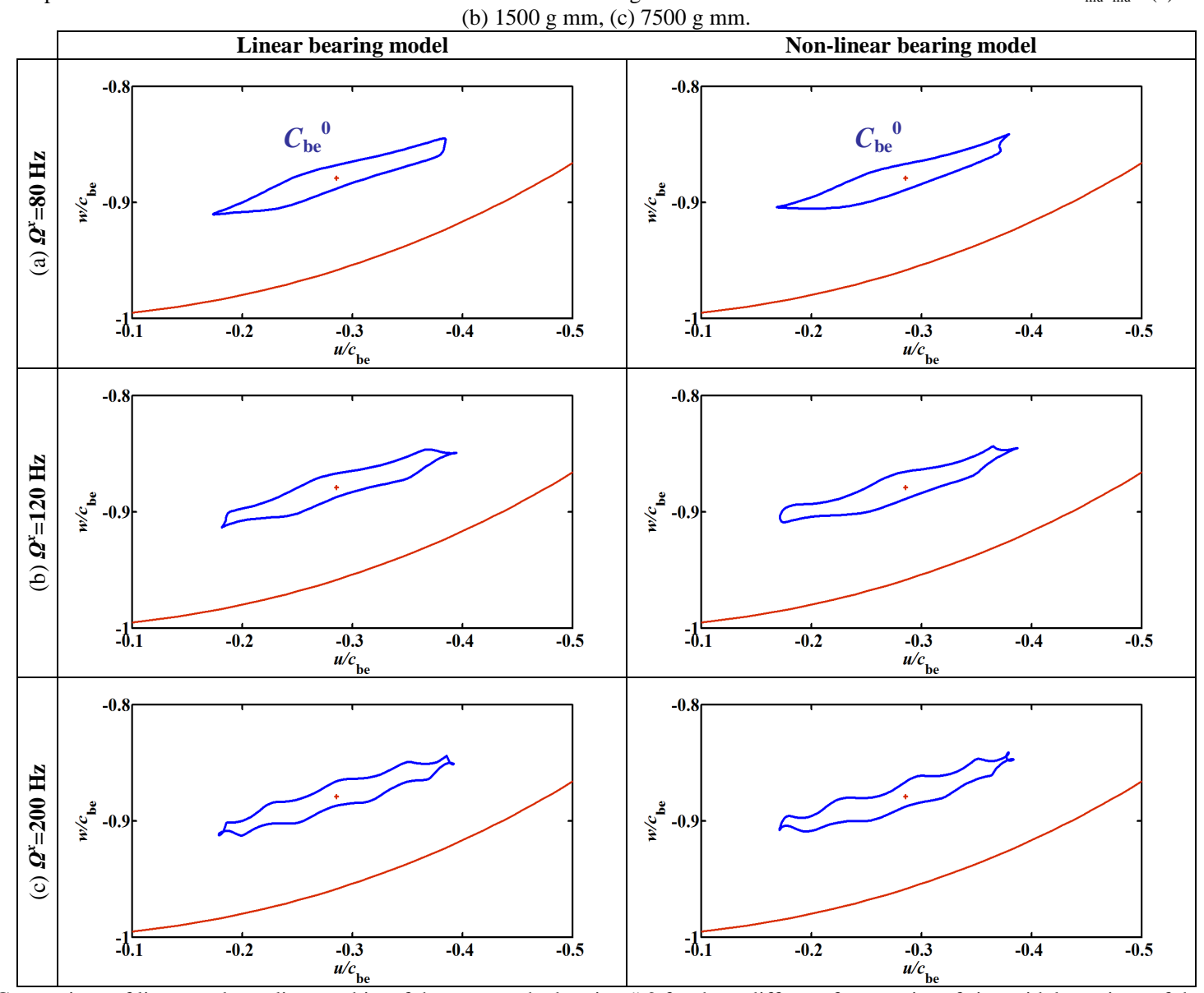

Fig. 9. Comparison of linear and non-linear orbits of the rotor at the bearing \# 2 for three different frequencies of sinusoidal rotations of the support: $\omega^{x}=\omega^{x, a} \cos \Omega^{x} t$ with $\omega^{x, a}=1 \times 10^{-2} \mathrm{rad} / \mathrm{s}$ and $\Omega^{x}=$ (a) $80 \mathrm{~Hz}$, (b) $120 \mathrm{~Hz}$, (c) $200 \mathrm{~Hz}$. 


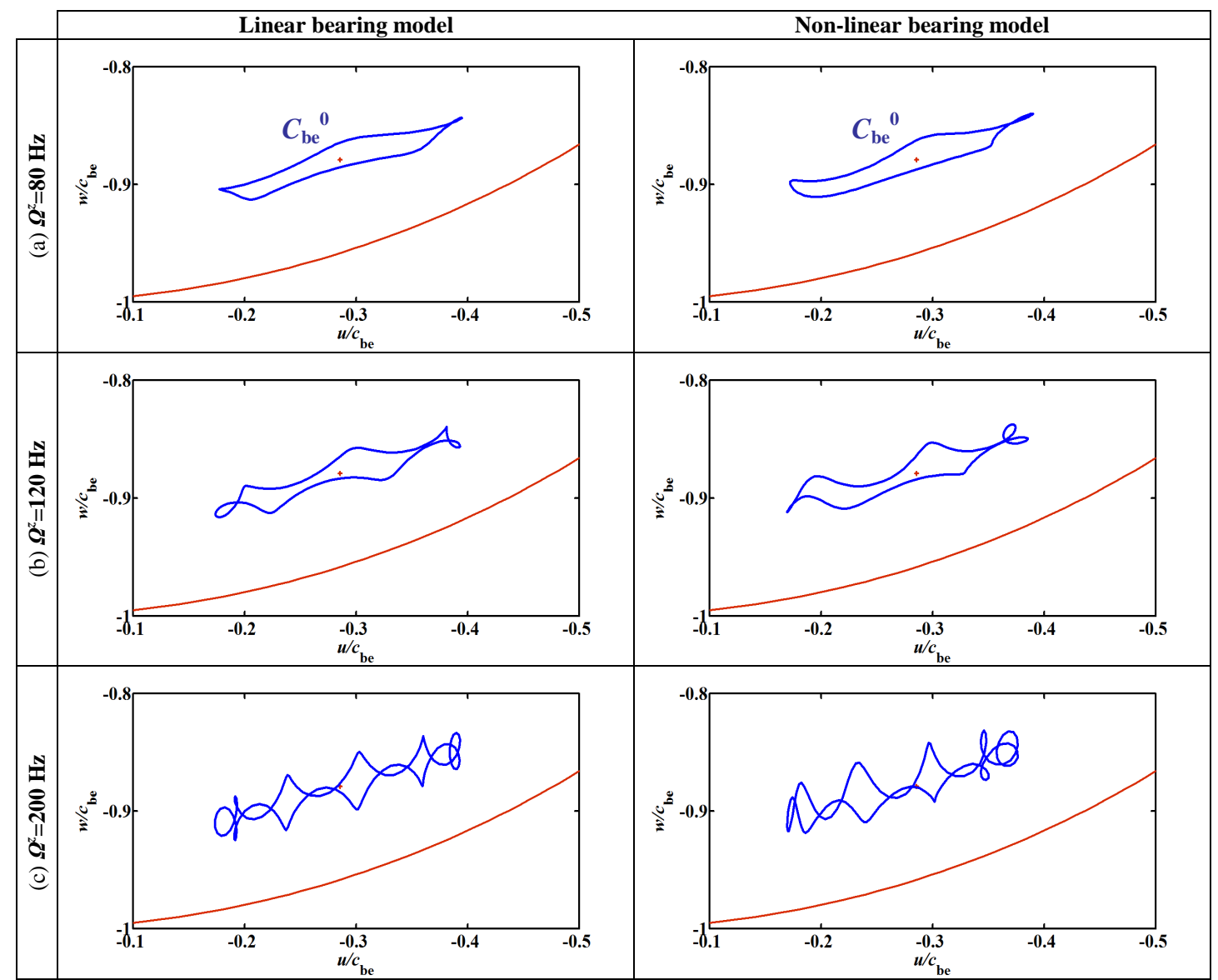

Fig. 10. Comparison of linear and non-linear orbits of the rotor at the bearing \# 2 for three different frequencies of sinusoidal translations of the support: $z_{O}=Z_{O} \cos \Omega^{z} t$ with $Z_{O}=1 \times 10^{-5} \mathrm{~m}$ and $\Omega^{z}=$ (a) $80 \mathrm{~Hz}$, (b) $120 \mathrm{~Hz}$, (c) $200 \mathrm{~Hz}$.

Unlike the previous section, the sinusoidal rotation of the support makes the orbits at the locations of the bearings different due to its normal and tangential accelerations. Focus is thus put on the bearing \# 2 which is more affected by the support rotation.

Since the excitations due to the mass unbalance and to the support motions are sinusoidal and commensurable, the external excitation of the rotor is harmonic of period $\tau_{\text {ex }}$ equal to the inverse of the greatest common factor of the mass unbalance frequency $\Omega$ and the support motion frequency $\Omega^{x}$ or $\Omega^{z}$. For example, one period of the first external excitation of the rotor corresponds to one period of the mass unbalance excitation $(\Omega=20 \mathrm{~Hz})$ and to four periods of the support motion for $\Omega^{x}$ or $\Omega^{z}=80 \mathrm{~Hz}$. In addition, the linear and non-linear orbits of the rotor in Figs. 9 and 10 are harmonic of periods $\tau_{\mathrm{r}, 1}$ and $\tau_{\mathrm{r}, \mathrm{ll}}$ equal to $\tau_{\mathrm{ex}}$. As expected when the amplitude of the support motions is small, the shape of the linear and non-linear orbits in Figs. 9 and 10 changes a lot with frequency and is made more complicated, while their magnitudes are slightly modified when compared to the orbits shown in Fig. 8(b) corresponding to the fixed support. Lastly, the linear and non-linear orbits are rather similar, i.e., the dynamic motion of the rotor remains close to the static equilibrium position $C_{\mathrm{be}}^{0}$ and the linear bearing model can still be considered to be acceptable.

The relative linear and non-linear time history responses in the $O z$ direction of the rotor at the bearing \# 2 due to the mass unbalance effect and to the sinusoidal rotational or translational excitations of the support are shown in Figs. 11 and 12 . They confirm the results provided in Figs. 9(c) and 10(c), i.e., these responses exhibit similar evolutions and their periods $\tau_{\mathrm{r}, 1}$ and $\tau_{\mathrm{r}, \mathrm{n}}$ are equal to the period $\tau_{\text {ex }}$ of the external excitation of the rotor. Moreover, the FFTs of the linear response (see Figs. 11(a) and 12(a)) show two frequency components due to the mass unbalance excitation $(20 \mathrm{~Hz})$ and the support motions $(200 \mathrm{~Hz})$, while the FFTs of the non-linear response (see Figs. 11(b) and 12(b)) show additional frequency components which correspond to multiples of $20 \mathrm{~Hz}$ (super-harmonics) relative to the harmonic motion of the rotor. The peaks at these additional frequencies are relatively small compared to those of $20 \mathrm{~Hz}$ and $200 \mathrm{~Hz}$. This validates the assumption of small displacements of the rotor in the vicinity of the static position $C_{\mathrm{be}}^{0}$.

\subsubsection{Influence of the support motion amplitude}

Figs. 13 and 14 introduce a comparison between linear orbits of the rotor and non-linear ones (obtained by the transient Newmark scheme and the shooting algorithm) at the bearing \# 2 located at node 9 for the mass unbalance excitation $\left(m_{\mathrm{mu}} r_{\mathrm{mu}}=1500 \mathrm{~g} \mathrm{~mm}\right)$ and the sinusoidal rotational or translational motions of the rotor support and due to the initial conditions $\boldsymbol{\delta}_{\mathrm{be}}^{0} / c_{\mathrm{be}}$. 
(a)

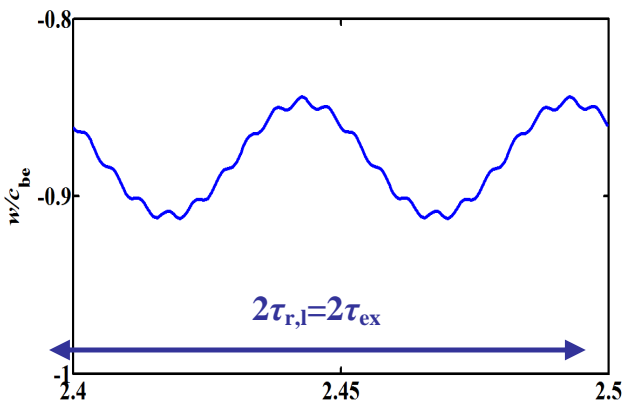

2.45
$t(s)$

(b)

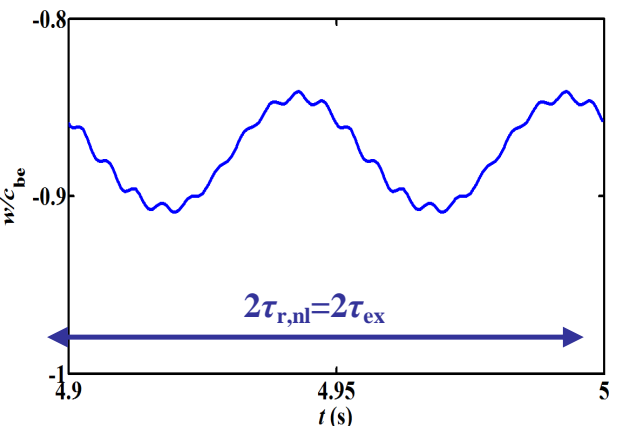

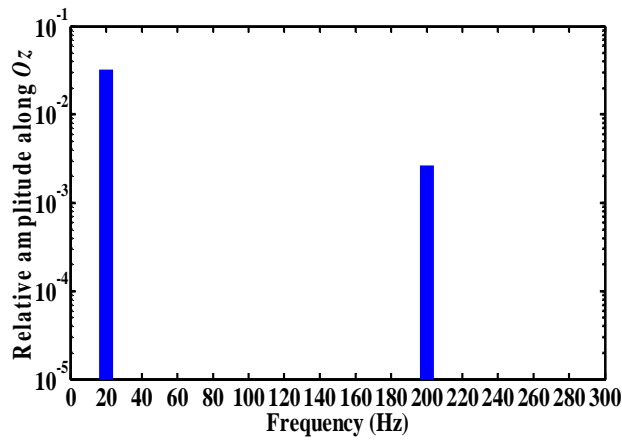

Frequency $(\mathrm{Hz})$

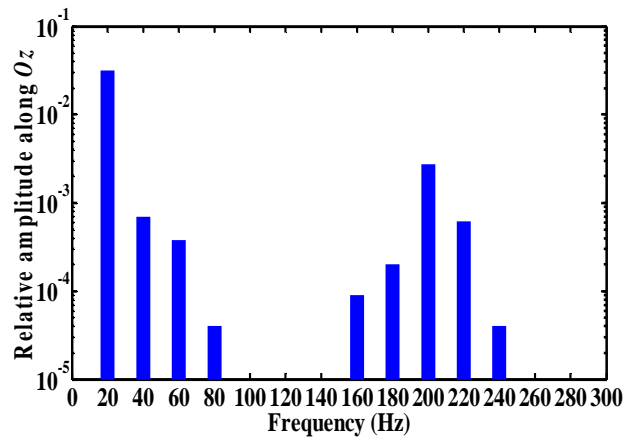

Fig. 11. (a) Linear, and (b) non-linear responses of the rotor and their FFTs at the bearing \# 2 due to a sinusoidal rotation of the support: $\omega^{x}=\omega^{x, a} \cos \Omega^{x} t$ with $\omega^{x, a}=1 \times 10^{-2} \mathrm{rad} / \mathrm{s}$ and $\Omega^{x}=200 \mathrm{~Hz}$.

(a)

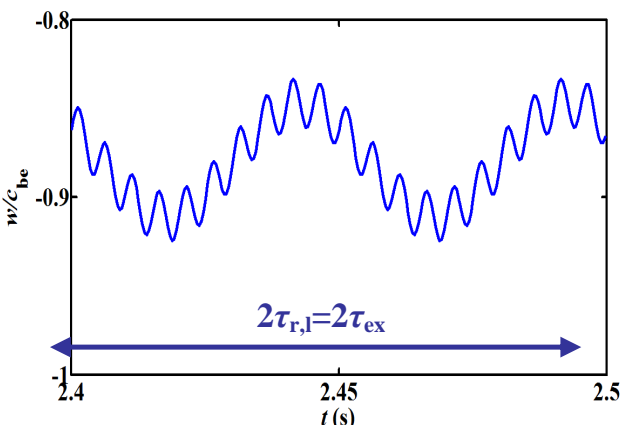

(b)

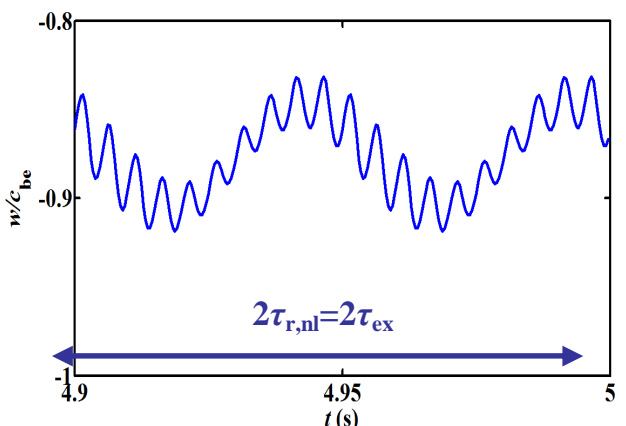

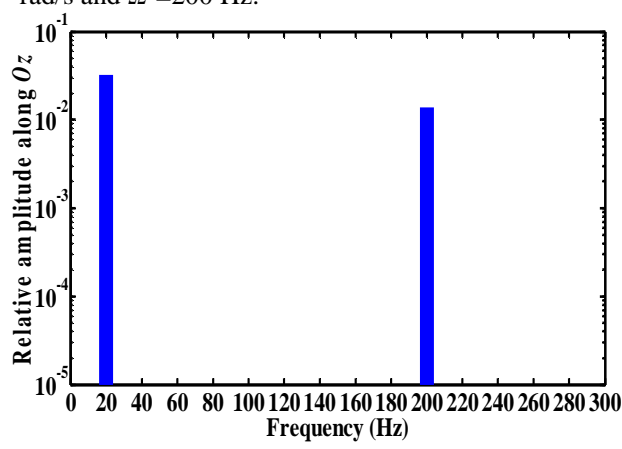

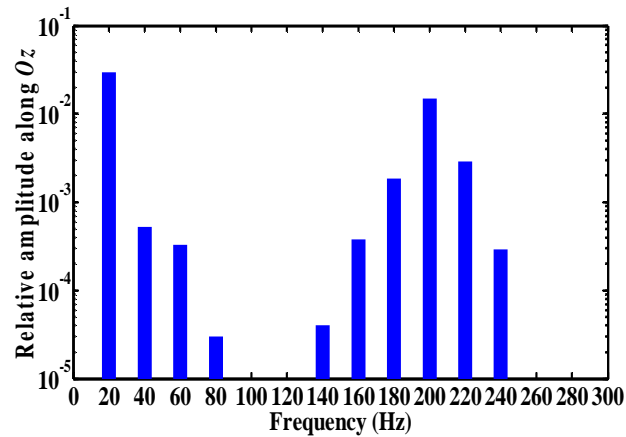

Fig. 12. (a) Linear, and (b) non-linear responses of the rotor and their FFTs at the bearing \# 2 due to a sinusoidal translation of the support: $z_{O}=Z_{O} \cos \Omega^{z} t$ with $Z_{O}=1 \times 10^{-5} \mathrm{~m}$ and $\Omega^{z}=200 \mathrm{~Hz}$.

In Fig. 13, the frequency of the support rotation is kept constant $\left(\Omega^{x}=80 \mathrm{~Hz}\right)$, while its amplitude varies $\left(\omega^{x, a}=8 \times 10^{-2} \mathrm{rad} / \mathrm{s}\right.$ and $\left.12 \times 10^{-2} \mathrm{rad} / \mathrm{s}\right)$, i.e., the corresponding angular displacement amplitudes of the support are $\omega^{x, a} / \Omega^{x}=1.59 \times 10^{-4} \mathrm{rad}$ and $2.39 \times 10^{-4} \mathrm{rad}$ respectively. In Fig. 14, the frequency of the support translation is kept constant $\left(\Omega^{z}=80 \mathrm{~Hz}\right)$, while its amplitude varies $\left(Z_{O}=3 \times 10^{-5} \mathrm{~m}\right.$ and $\left.5 \times 10^{-5} \mathrm{~m}\right)$.

As explained previously, the excitations due to the mass unbalance and to the support motions are sinusoidal and commensurable. Thus the external excitation of the rotor is harmonic of period $\tau_{\text {ex }}$ equal to the inverse of the greatest common factor of the mass unbalance frequency $\Omega$ and the support motion frequency $\Omega^{x}$ or $\Omega^{z}$. In the case where $\omega^{x, a}=8 \times 10^{-2} \mathrm{rad} / \mathrm{s}$ and $Z_{O}=3 \times 10^{-5} \mathrm{~m}$, see Figs. 13(a) and 14(a), the linear and non-linear orbits of the rotor are harmonic of periods $\tau_{\mathrm{r}, \mathrm{l}}$ and $\tau_{\mathrm{r}, \mathrm{nl}}$ equal to the period $\tau_{\mathrm{ex}}$ but they are not similar. This justifies that the assumption of small displacements of the rotor in the vicinity of the static equilibrium position $C_{\mathrm{be}}^{0}$ is not verified anymore and the validity range of the linearized bearing model is more affected by the amplitude than by the frequency of the support motion. For larger amplitudes of the support motions $\omega^{x, a}=12 \times 10^{-2} \mathrm{rad} / \mathrm{s}$ and $Z_{O}=5 \times 10^{-5} \mathrm{~m}$, the non-linear 
bearing model yields a very complex orbit with a change of dynamic regime, i.e., the appearance of a bifurcation which corresponds to a period-doubling orbit with $\tau_{\mathrm{r}, \mathrm{nl}}=2 \tau_{\mathrm{ex}}$, see Figs. 13(b) and 14(b). It can be noted that this period-doubling motion is also obtained when the transient time integration algorithm is used as a checking procedure. As expected, the linear bearing model is unable to reproduce this period-doubling motion, i.e., the linear motion of the rotor remains $1 \tau_{\mathrm{ex}}$-periodic.

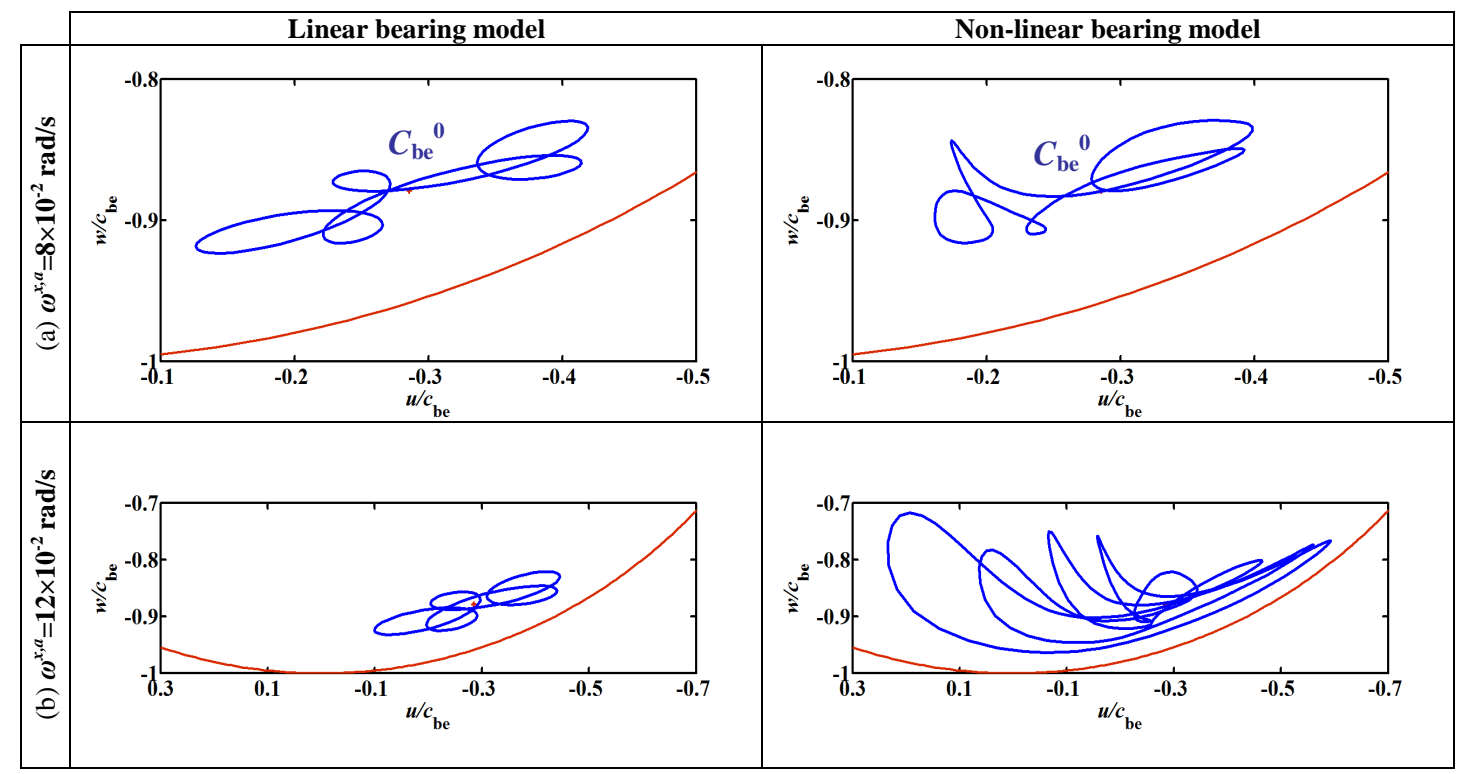

Fig. 13. Comparison of linear and non-linear orbits of the rotor at the bearing \# 2 for two different amplitudes of sinusoidal rotations of the support: $\omega^{x}=\omega^{x, a} \cos \Omega^{x} t$ with $\Omega^{x}=80 \mathrm{~Hz}$ and $\omega^{x, a}=$ (a) $8 \times 10^{-2} \mathrm{rad} / \mathrm{s}$, (b) $12 \times 10^{-2} \mathrm{rad} / \mathrm{s}$.

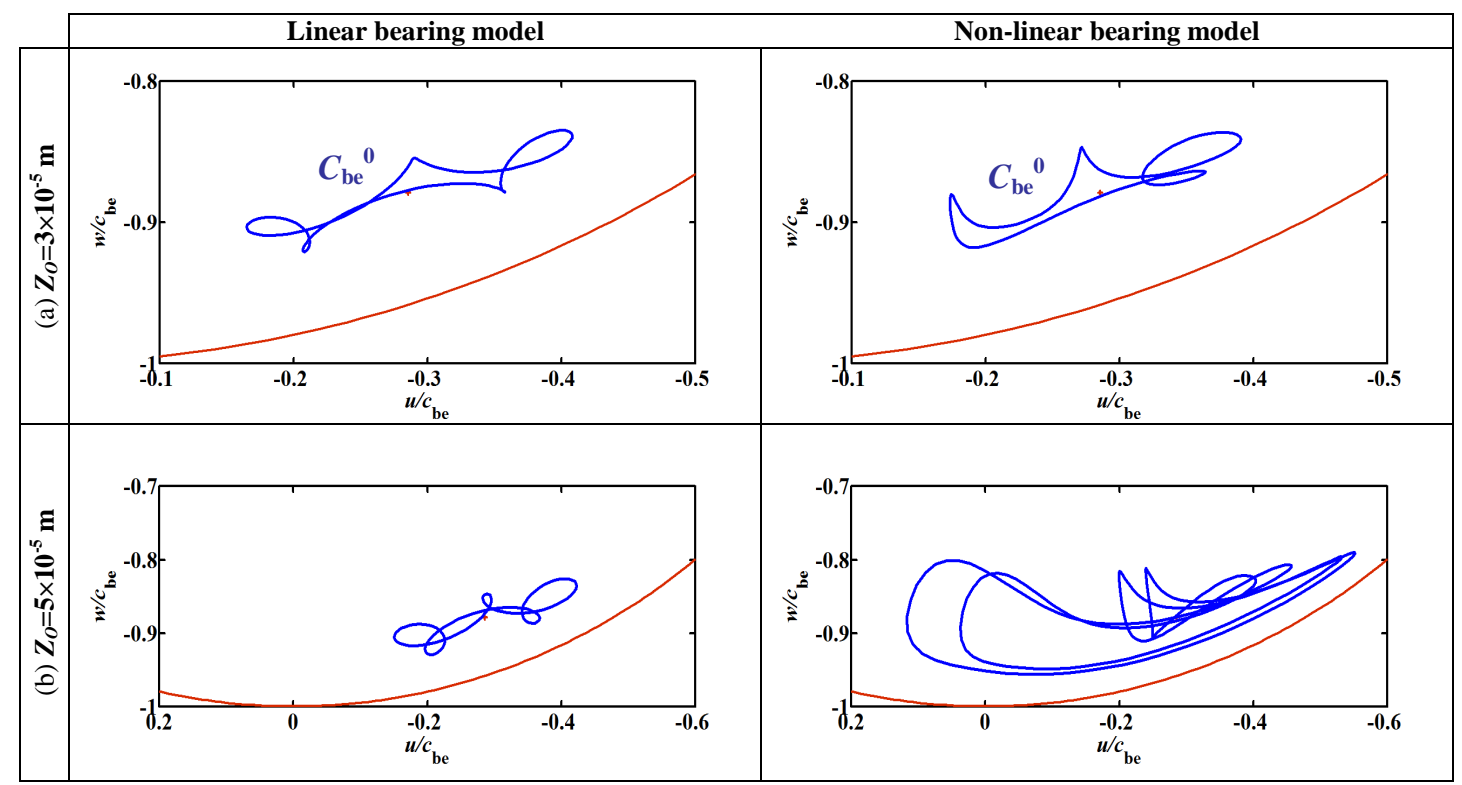

Fig. 14. Comparison of linear and non-linear orbits of the rotor at the bearing \# 2 for two different amplitudes of sinusoidal translations of the support: $z_{O}=Z_{O} \cos \Omega^{z} t$ with $\Omega^{z}=80 \mathrm{~Hz}$ and $Z_{O}=$ (a) $3 \times 10^{-5} \mathrm{~m}$, (b) $5 \times 10^{-5} \mathrm{~m}$.

Figs. 15 and 16 illustrate the linear and non-linear time history responses along the $O z$ axis of the shaft geometric center within the bearing \# 2 for the mass unbalance effect and the sinusoidal rotational or translational excitations of the support. They verify the results pointed out in Figs. 13(b) and 14(b), i.e., the non-linear response is $2 \tau_{\mathrm{ex}}$-periodic because its period $\tau_{\mathrm{r}, \mathrm{nl}}$ is two times higher than the period $\tau_{\mathrm{r}, 1}$ of the linear response. In addition, the FFTs of the linear response (see Figs. 15(a) and 16(a)) display two frequency components due to the mass unbalance $(20 \mathrm{~Hz})$ and the support motions $(80 \mathrm{~Hz})$, while the FFTs of the non-linear response (see Figs. 15(b) and 16(b)) highlight much more frequency components and contain harmonics, sub-harmonics and superharmonics relative to the period-doubling motion of the rotor.

Fig. 17 gives the linear and non-linear orbits of the rotor within the bearing \# 1 (node 1) due to the mass unbalance excitation ( $\left.m_{\mathrm{mu}} r_{\mathrm{mu}}=1500 \mathrm{~g} \mathrm{~mm}\right)$ combined with the sinusoidal rotation $\left(\omega^{x, a}=12 \times 10^{-2} \mathrm{rad} / \mathrm{s}\right.$ and $\left.\Omega^{x}=80 \mathrm{~Hz}\right)$ of the support. This figure confirms that the orbits at both bearings are very different (see Fig. 13(b)) in the case of the support rotation due to its normal and tangential 
accelerations. From Fig. 17(b), it can be noticed that the non-linear orbit in the bearing \# 1 is also $2 \tau_{\text {ex }}$-periodic. This demonstrates the non-linear couplings between the localized degrees of freedom of the hydrodynamic bearings through the shaft.

(a)

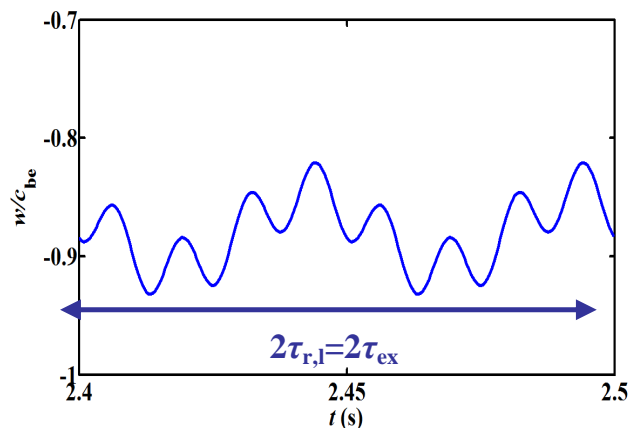

(b)

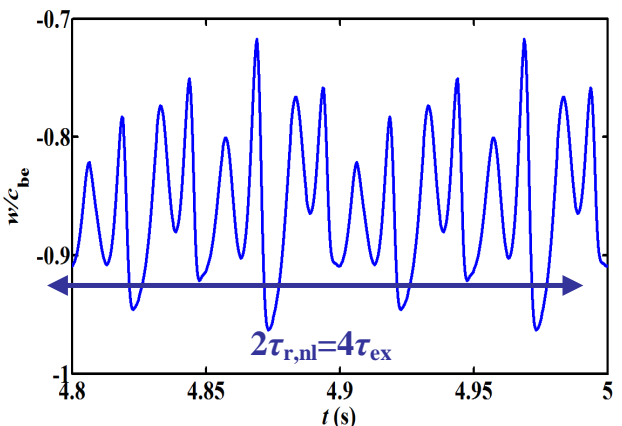

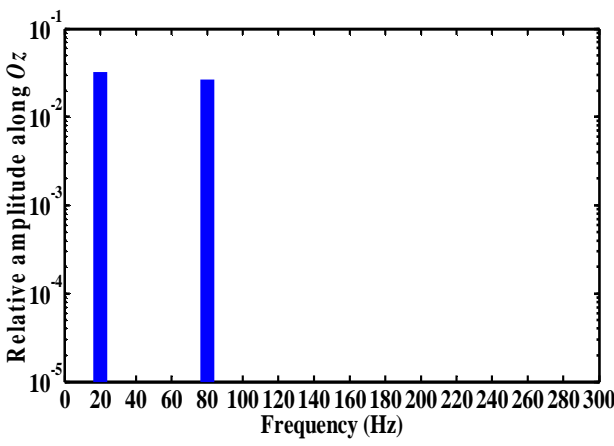

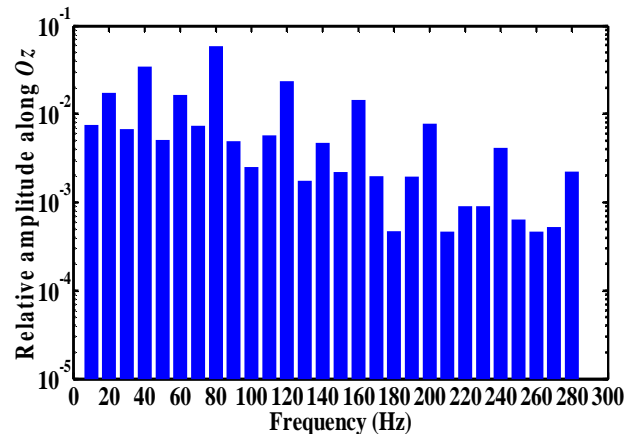

Fig. 15. (a) Linear, and (b) non-linear responses of the rotor and their FFTs at the bearing \# 2 due to a sinusoidal rotation of the support: $\omega^{x}=\omega^{x, a} \cos \Omega^{x} t$ with $\omega^{x, a}=12 \times 10^{-2} \mathrm{rad} / \mathrm{s}$ and $\Omega^{x}=80 \mathrm{~Hz}$.

(a)

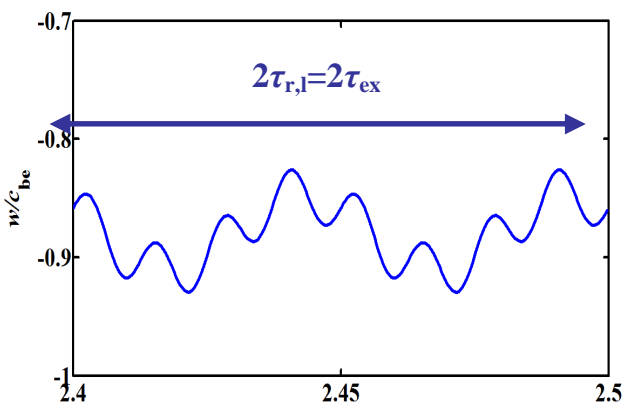

$t(\mathrm{~s})$

(b)

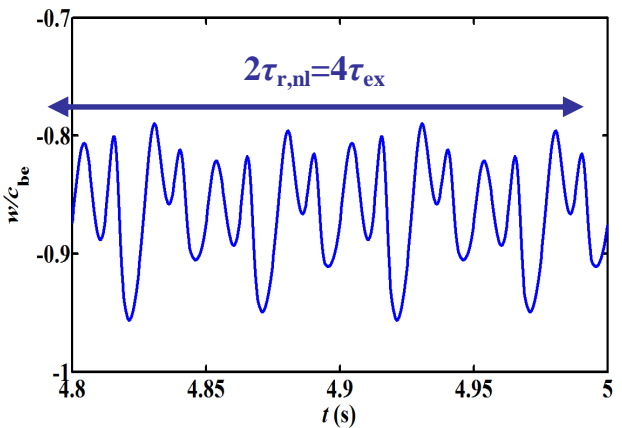

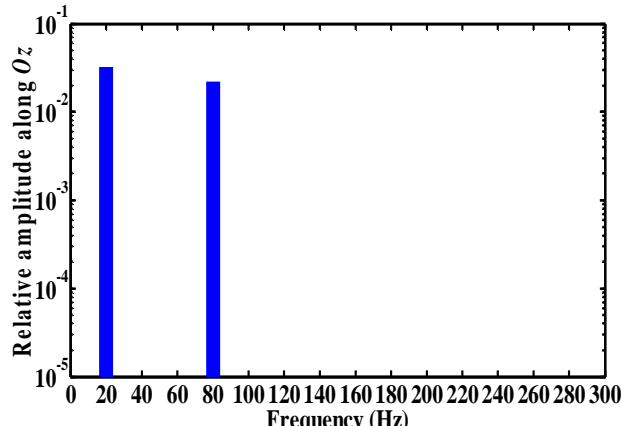

Frequency $(\mathrm{Hz})$

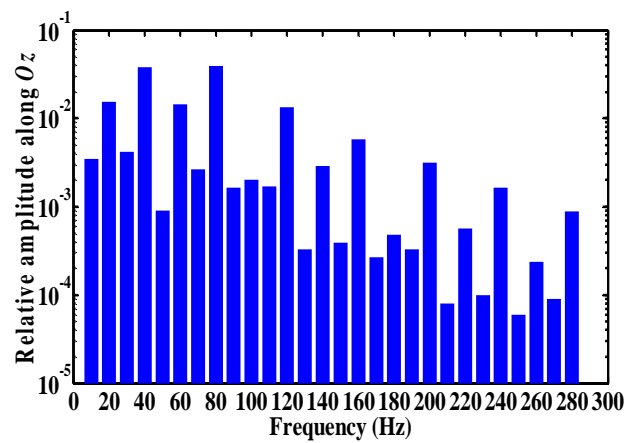

Fig. 16. (a) Linear, and (b) non-linear responses of the rotor and their FFTs at the bearing \# 2 due to a sinusoidal translation of the support: $z_{O}=Z_{O} \cos \Omega^{z} t$ with $Z_{O}=5 \times 10^{-5} \mathrm{~m}$ and $\Omega^{z}=80 \mathrm{~Hz}$.

(a)

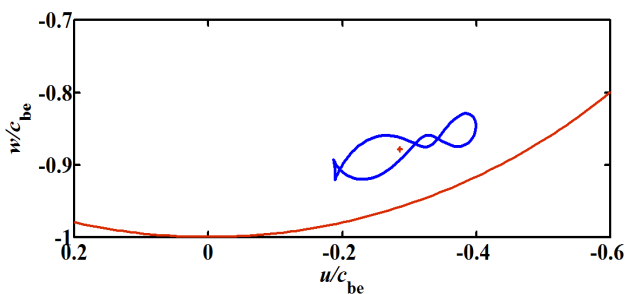

(b)

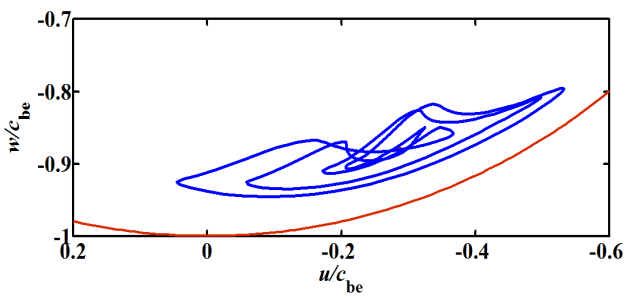

Fig. 17. (a) Linear, and (b) non-linear orbits of the rotor at the bearing \# 1 in the presence of a sinusoidal rotation of the support: $\omega^{x}=\omega^{x, a} \cos \Omega^{x} t$ with $\omega^{x, a}=12 \times 10^{-2} \mathrm{rad} / \mathrm{s}$ and $\Omega^{x}=80 \mathrm{~Hz}$. 


\subsection{Route to chaos in the rotor-non-linear hydrodynamic bearing system}

\subsubsection{Bifurcation diagrams}

The response of the linear rotor system to a harmonic external excitation of period $\tau_{\mathrm{ex}}$ is harmonic of same period, i.e., $\tau_{\mathrm{r}}=\tau_{\mathrm{ex}}$, while the corresponding response of the non-linear rotor system can be non-periodic or can have a different period due to bifurcations as seen in Section 6.3.3. Such changes in the non-linear dynamic regime of the system can be investigated by means of diagrams of bifurcation of periodic solution.

Figs. 18 and 19 present the bifurcation diagrams of the rotor at the bearing \# 2 (node 9) for the mass unbalance effect $\left(m_{\mathrm{mu}} r_{\mathrm{mu}}=1500 \mathrm{~g} \mathrm{~mm}\right)$ and the sinusoidal rotational or translational motions of the support of frequency $\Omega^{x}$ or $\Omega^{z}=80 \mathrm{~Hz}$. For each diagram, the amplitudes $\omega^{x, a}$ or $Z_{O}$ of the support motions are used as bifurcation parameters to provide essential information about the Poincaré sections. The steps used for the bifurcation parameters $\omega^{x, a}$ and $Z_{O}$ are $0.05 \times 10^{-2} \mathrm{rad} / \mathrm{s}$ and $0.05 \times 10^{-5} \mathrm{~m}$ respectively. The period of the harmonic external excitation of the rotor is $\tau_{\mathrm{ex}}=1 / 20 \mathrm{~s}=0.05 \mathrm{~s}$ which corresponds to the inverse of the greatest common factor of the mass unbalance frequency $\Omega=20 \mathrm{~Hz}$ and the support motion frequency $\Omega^{x}$ or $\Omega^{z}=80 \mathrm{~Hz}$. The transient numerical simulations are performed with 1000 rotor revolutions (i.e., $1000 \tau_{\mathrm{ex}}$ ) and 512 time steps per period $\tau_{\mathrm{ex}}$. The data of the first 500 revolutions of the rotor are not used in the bifurcation diagrams in order to exclude the transient regime. The Poincaré sections are obtained by taking successive intersections of the $x$ coordinates of the dynamic displacements of the rotor with the instants corresponding to the multiples of the period $\tau_{\mathrm{ex}}$, i.e., $t=k \tau_{\mathrm{ex}}$ and $k \in[501 ; 1000]$. The initial conditions for the transient simulations are set to $\boldsymbol{\delta}_{\mathrm{be}}^{0} / c_{\mathrm{be}}$. Lastly, Tables 3 and 4 present a summary of the different dynamic regimes observed on the bifurcation diagrams of the on-board rotor-bearing system excited by sinusoidal rotational or translational motions of its support as a function of the bifurcation parameters.

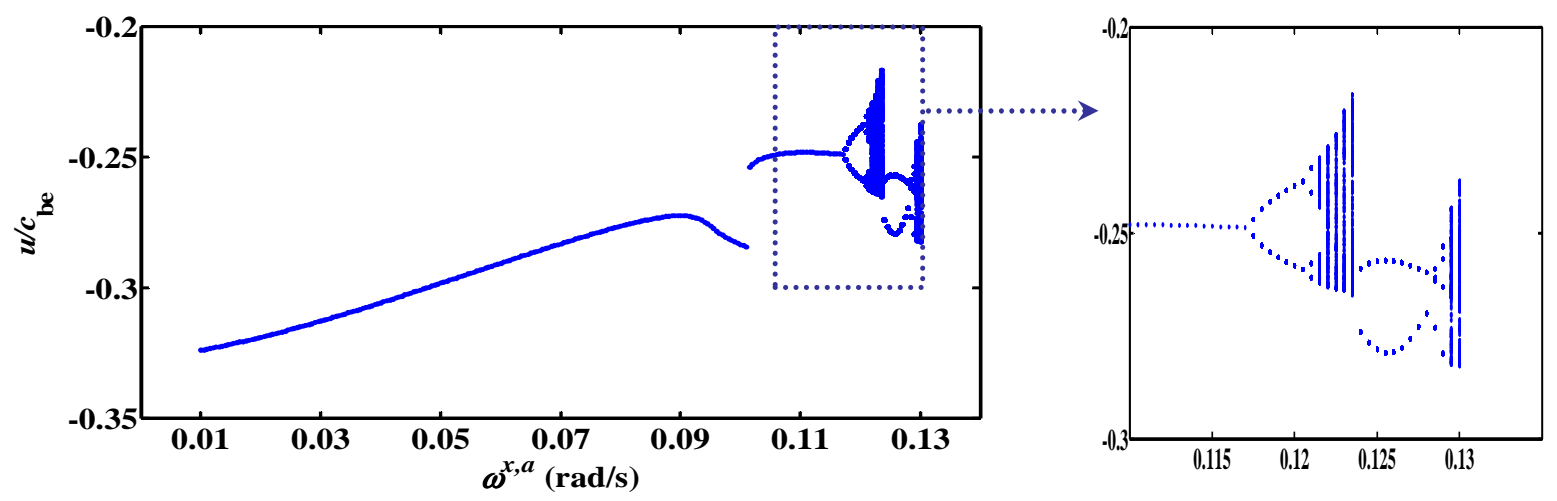

Fig. 18. Bifurcation diagram of the rotor at the bearing \# 2 for a sinusoidal rotation of the support: $\omega^{x}=\omega^{x, a} \cos \Omega^{x} t$ with $\Omega^{x}=80 \mathrm{~Hz}$.

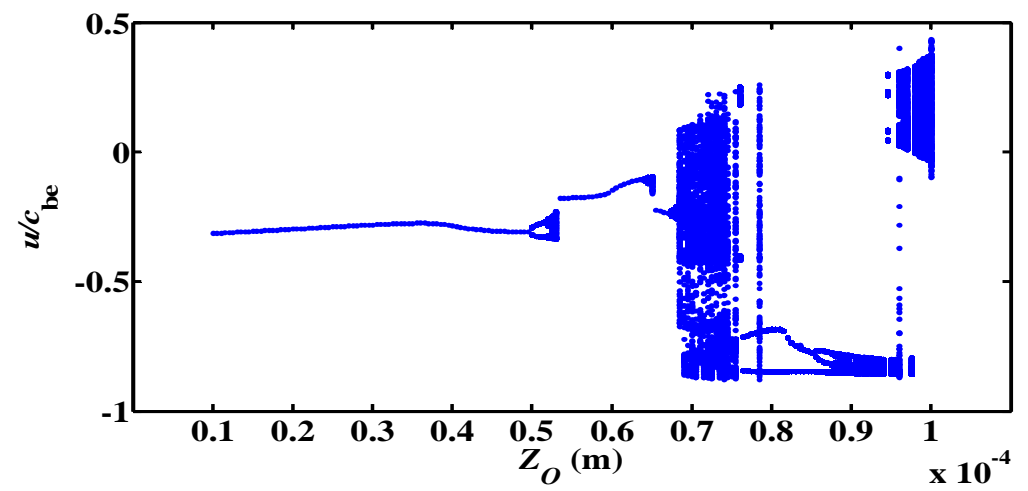

Fig. 19. Bifurcation diagram of the rotor at the bearing \# 2 for a sinusoidal translation of the support: $z_{O}=Z_{O} \cos \Omega^{z} t$ with $\Omega^{z}=80 \mathrm{~Hz}$.

Table 3. Types of motion identified in the bifurcation diagram due to the sinusoidal rotation of the support.

\begin{tabular}{ll}
\hline Type of the rotor motion & Support rotation amplitude $\omega^{x, a}\left(10^{-2} \mathrm{rad} / \mathrm{s}\right)$ \\
\hline $1 \tau_{\mathrm{ex}}$-periodic & {$[1-11.7]$} \\
\hline $2 \tau_{\mathrm{ex}}$-periodic & {$[11.75-12.05],[12.4-12.8]$} \\
\hline $4 \tau_{\mathrm{ex}}$-periodic & {$[12.1],[12.85]$} \\
\hline Quasi-periodic & {$[12.15-12.35],[12.9-13]$} \\
\hline
\end{tabular}


Table 4. Types of motion identified in the bifurcation diagram due to the sinusoidal translation of the support.

\begin{tabular}{ll}
\hline Type of the rotor motion & Support translation amplitude $Z_{o}\left(10^{-5} \mathrm{~m}\right)$ \\
\hline $1 \tau_{\mathrm{ex}}$-periodic & {$[1-4.95],[5.35-6.3],[6.55-6.7]$} \\
\hline $2 \tau_{\mathrm{ex}}$-periodic & {$[5-5.1],[6.35-6.4],[6.75],[7.65-7.8]$,} \\
\hline $4 \tau_{\mathrm{ex}}$-periodic & {$[7.9-8.3],[8.4-8.5]$} \\
\hline $8 \tau_{\mathrm{ex}}$-periodic & {$[8.55-8.7]$} \\
\hline $16 \tau_{\mathrm{ex}}$-periodic & {$[8.75],[9.45]$} \\
\hline Quasi-periodic & {$[8.8]$} \\
\hline Chaotic & {$[5.15-5.3],[6.45-6.5],[6.8],[7.5],[7.6]$,} \\
\hline
\end{tabular}
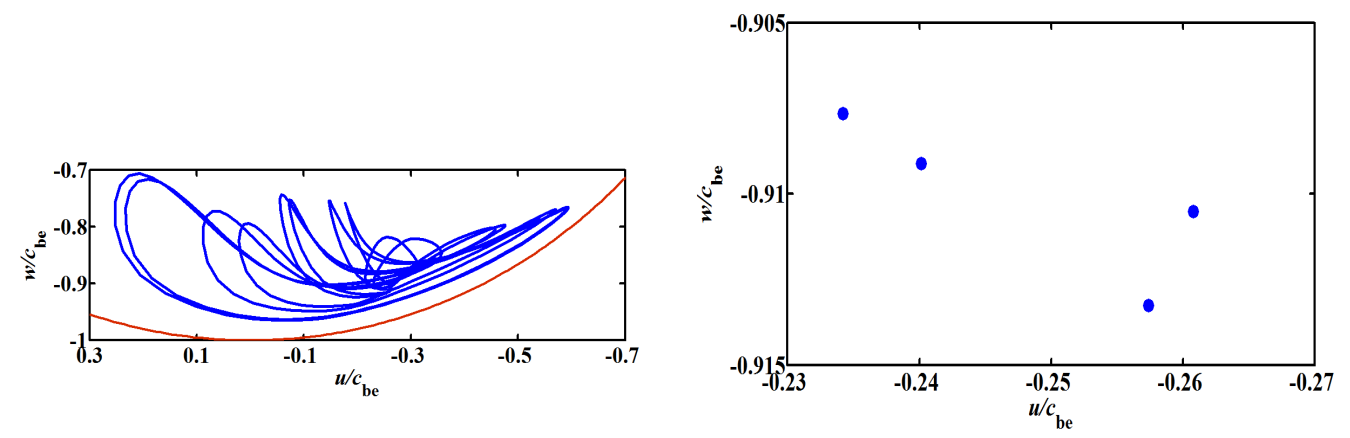

(a)

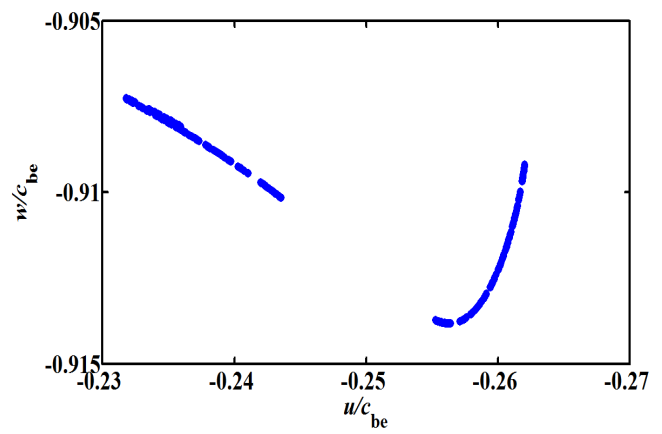

(b)
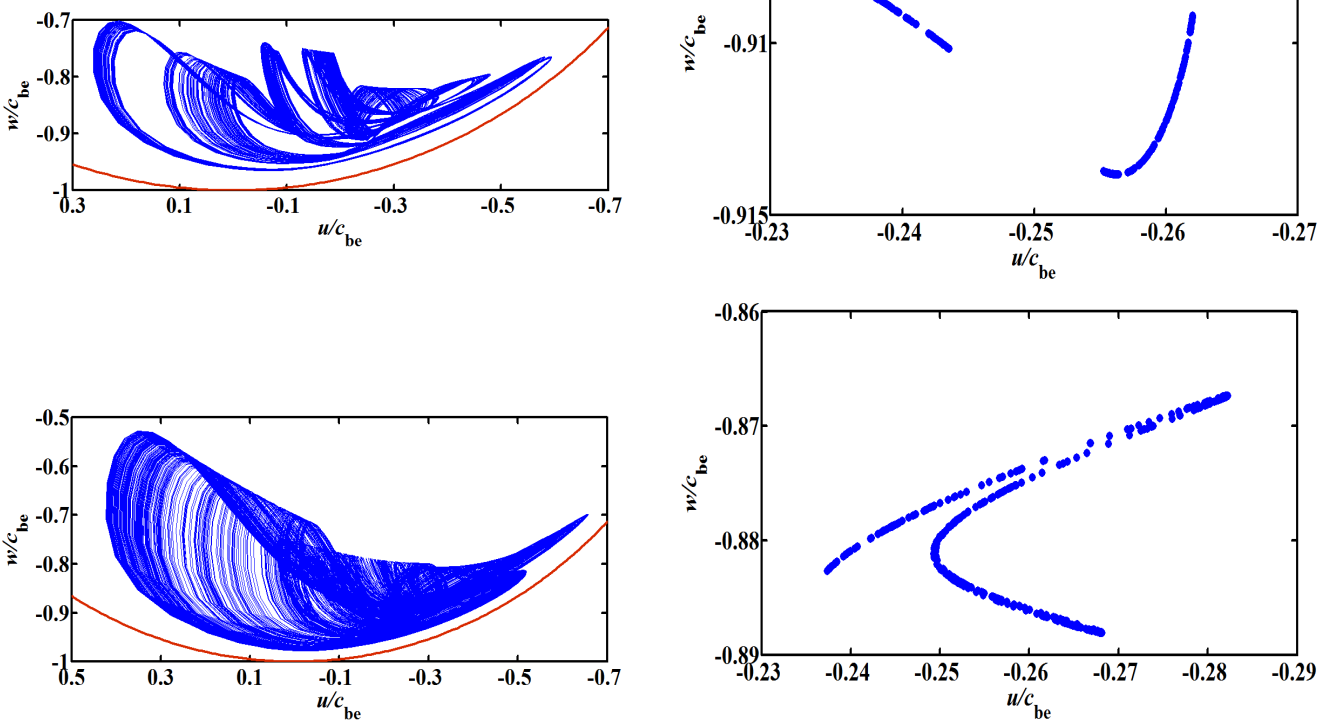

(c)

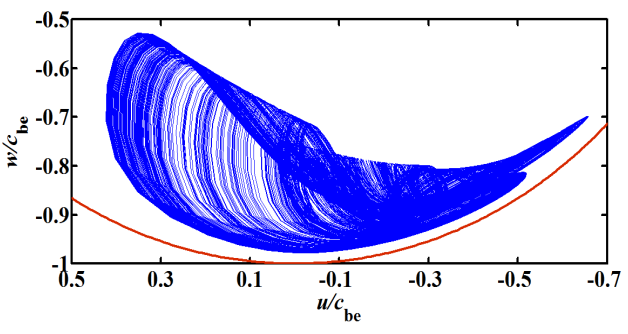

Fig. 20. Orbits and Poincaré maps of the rotor at the bearing \# 2 due to sinusoidal rotations of the support: $\omega^{x}=\omega^{x, a} \cos \Omega^{x} t$ with $\Omega^{x}=80 \mathrm{~Hz}$ and $\omega^{x, a}=(\mathrm{a})$ $12.1 \times 10^{-2} \mathrm{rad} / \mathrm{s}$, (b) $12.15 \times 10^{-2} \mathrm{rad} / \mathrm{s}$, (c) $13 \times 10^{-2} \mathrm{rad} / \mathrm{s}$.

\subsubsection{Sub-harmonic, quasi-periodic and chaotic bifurcation responses}

In this section, some orbits of the rotor and their projected Poincaré maps at the bearing \# 2 (node 9) corresponding to values of interest of the bifurcation parameters $\omega^{x, a}$ or $Z_{o}$ of the bifurcation diagrams in Figs. 18 and 19 are presented. The relative coordinates of the static position of the shaft center in the bearings $\boldsymbol{\delta}_{\mathrm{be}}^{0} / c_{\mathrm{be}}$ are used as initial conditions for the transient dynamic computations. The Poincaré maps are produced by considering successive intersections of the relative dynamic displacements of the rotor with the instants associated with the multiples of the period $\tau_{\mathrm{ex}}=0.05 \mathrm{~s}$ of the harmonic external excitation of the rotor starting from $t=501 \tau_{\mathrm{ex}}$ to get rid of the transient regime.

When the amplitudes $\omega^{x, a}$ of the support rotation are successively equal to $12.1 \times 10^{-2} \mathrm{rad} / \mathrm{s}$ (Fig. 20(a)), $12.15 \times 10^{-2} \mathrm{rad} / \mathrm{s}$ (Fig. $20(\mathrm{~b}))$ and $13 \times 10^{-2} \mathrm{rad} / \mathrm{s}$ (Fig. 20(c)), the rotor orbits highlight a sub-harmonic motion $\left(4 \tau_{\text {ex }}\right.$-periodic) and quasi-periodic motions respectively. When the amplitudes $Z_{O}$ of the support translation are successively equal to $7.3 \times 10^{-5} \mathrm{~m}$ (Fig. 21 (a)), $8.6 \times 10^{-5} \mathrm{~m}$ (Fig. $21(\mathrm{~b}))$ and $9.5 \times 10^{-5} \mathrm{~m}$ (Fig. $21(\mathrm{c})$ ), the rotor orbits display a chaotic motion, a sub-harmonic motion ( $4 \tau_{\mathrm{ex}}$-periodic) and a quasiperiodic motion respectively. 
(a)
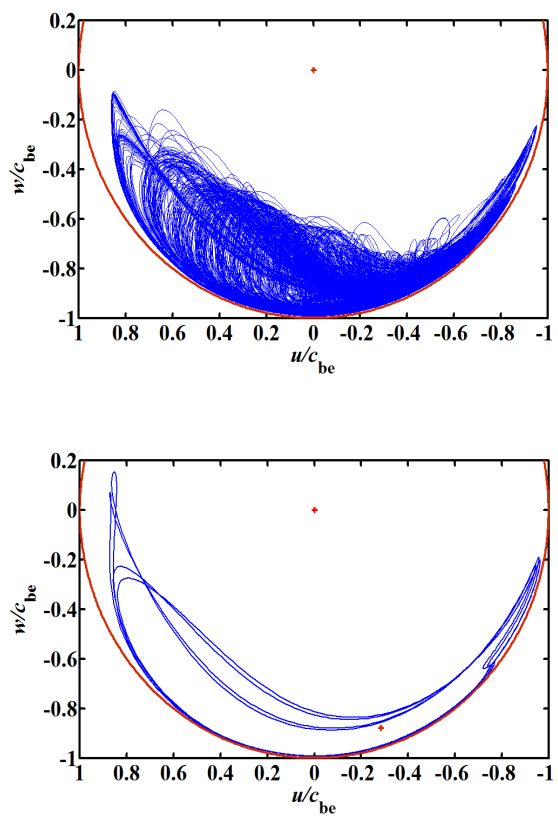

(b)

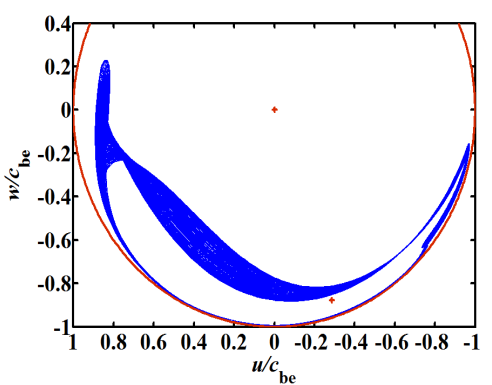

(c)
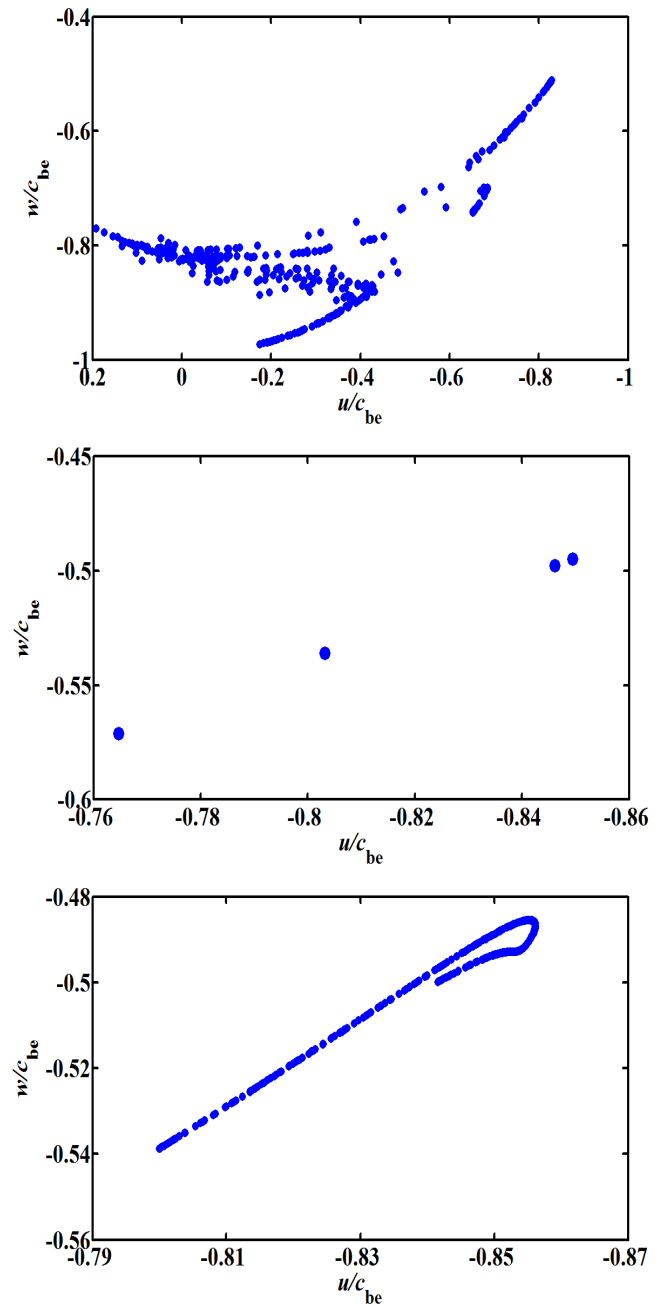

Fig. 21. Orbits and Poincaré maps of the rotor at the bearing \# 2 due to sinusoidal translations of the support: $z_{O}=Z_{O} \cos \Omega^{z} t$ with $\Omega^{z}=80 \mathrm{~Hz}$ and $Z_{O}=$ (a) $7.3 \times 10^{-5} \mathrm{~m}$, (b) $8.6 \times 10^{-5} \mathrm{~m}$, (c) $9.5 \times 10^{-5} \mathrm{~m}$.

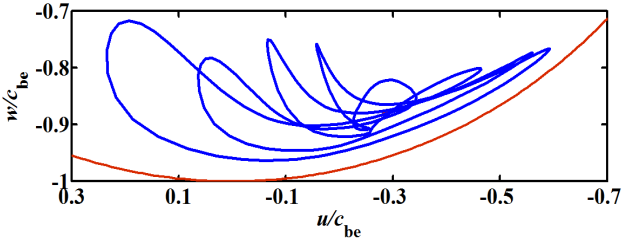

(a)
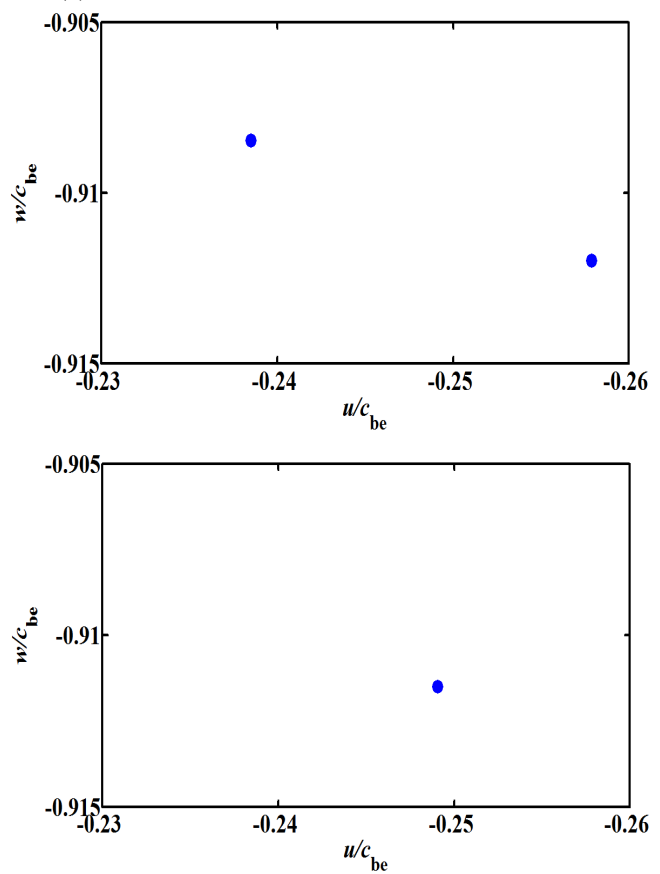

(b)

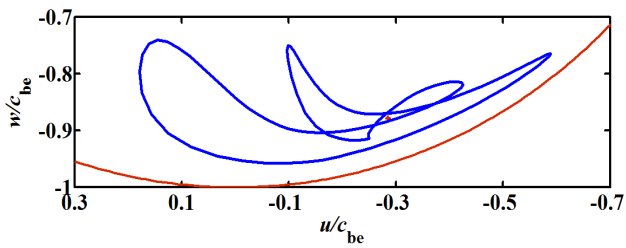

Fig. 22. Influence of the initial conditions on the orbits of the rotor in the presence of a mass unbalance $\left(m_{\mathrm{mu}} r_{\mathrm{mu}}=1500 \mathrm{~g}\right.$ mm) and sinusoidal rotations of the support: $\omega^{x}=\omega^{x, a} \cos \Omega^{x} t$ with $\omega^{x, a}=12 \times 10^{-2} \mathrm{rad} / \mathrm{s}$ and $\Omega^{x}=80 \mathrm{~Hz}$. 

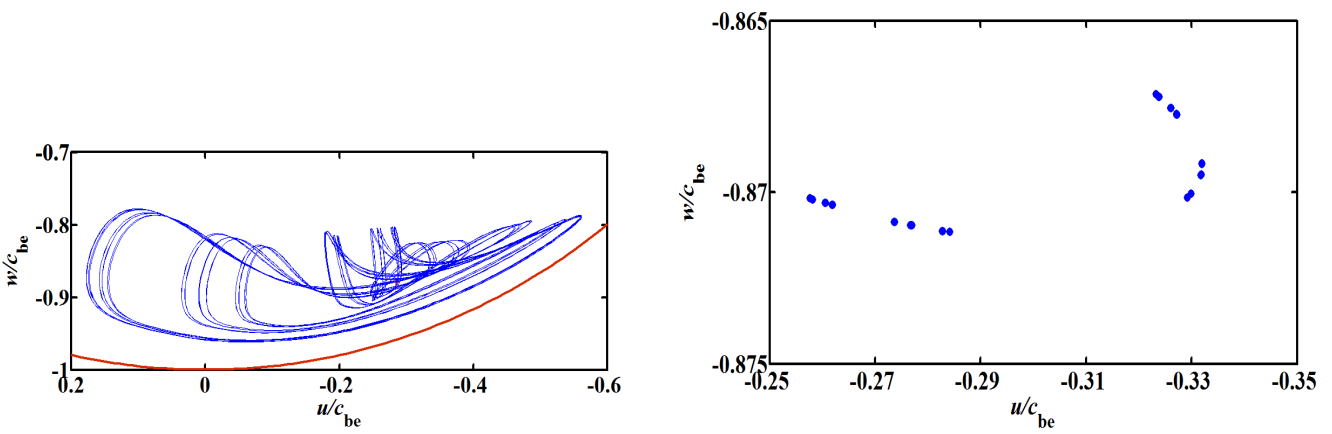

(a)

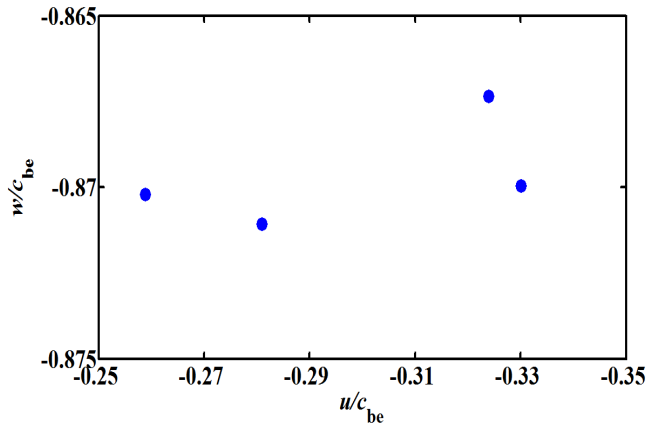

(b)

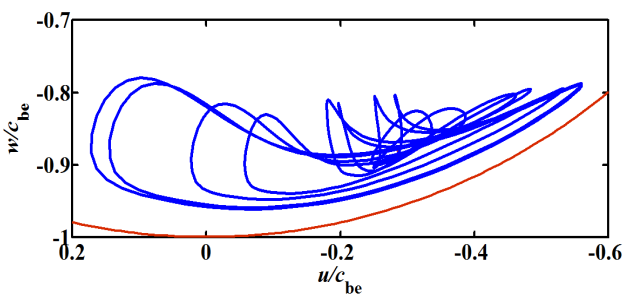

Fig. 23. Influence of the initial conditions on the orbits of the rotor in the presence of a mass unbalance $\left(m_{\mathrm{mu}} r_{\mathrm{mu}}=1500 \mathrm{~g} \mathrm{~mm}\right)$ and sinusoidal translations of the support: $z_{o}=Z_{O} \cos \Omega^{z} t$ with $Z_{O}=5.2 \times 10^{-5} \mathrm{~m}$ and $\Omega^{z}=80 \mathrm{~Hz}$.

\subsubsection{Sensitivity to initial conditions}

A very small change in the initial conditions for a non-linear rotor system can make an important change in its corresponding dynamic trajectory. This phenomenon is called "sensitivity to initial conditions" and is one of the main ways in which a non-linear system can be recognized. This is demonstrated in Figs. 22 and 23 which provide different orbits of rotor and their projected Poincaré maps at the bearing \# 2 (node 9) produced due to different initial conditions for the transient dynamic motion.

In Fig. 22, the rotor support is excited by a sinusoidal rotation of amplitude $\omega^{x, a}=12 \times 10^{-2} \mathrm{rad} / \mathrm{s}$ and frequency $\Omega^{x}=80 \mathrm{~Hz}$. The rotor orbit corresponds to a sub-harmonic motion ( $2 \tau_{\text {ex }}$-periodic), see Fig. 22(a), when the initial conditions are set to the coordinates of the static equilibrium position $\delta_{\mathrm{be}}^{0} / c_{\mathrm{be}}=[-0.29,-0.88]_{R}^{\mathrm{T}}$, while it corresponds to a harmonic motion $\left(1 \tau_{\mathrm{ex}}-\right.$ periodic), see Fig. 22(b), when the initial conditions are slightly modified and set to the coordinates $[-0.25,-0.91]_{R}^{\mathrm{T}}$.

In Fig. 23, the rotor support is excited by a sinusoidal translation of amplitude $Z_{O}=5.2 \times 10^{-5} \mathrm{~m}$ and frequency $\Omega^{z}=80 \mathrm{~Hz}$. The rotor orbit corresponds to a quasi-periodic motion, see Fig. 23(a), when the initial conditions are set to $\boldsymbol{\delta}_{\text {be }}^{0} / c_{\mathrm{be}}=[-0.29,-0.88]_{R}^{\mathrm{T}}$, while it corresponds to a sub-harmonic motion ( $4 \tau_{\text {ex }}$-periodic), see Fig. 23(b), when the initial conditions are set to $[-0.26,-0.87]_{R}^{\mathrm{T}}$.

\section{Conclusions}

In order to predict and analyze numerically the role of the sinusoidal rotational or translational motions of the support in the dynamic behavior of an on-board rotor-non-linear hydrodynamic journal bearing system, a new finite element model based on the Timoshenko beam theory is presented in this paper. This model includes the effects corresponding to the rotary inertia, the gyroscopic inertia, the shear deformation of shaft, the geometric asymmetry of shaft and/or rigid disk as well as six types of deterministic motions (rotations and translations) of the rotor rigid support. In addition, the rotational effects and the geometric stiffening effects associated with the centrifugal stressing caused by the support rotations are taken into consideration.

The derivation of the equations of motion displays that the rotational motions of the rotor support bring not only a parametric contribution in the damping and stiffness matrices of the rotor but also additional external force vectors, while the translational motions of the support only add external force vectors. A robust algorithm combining the Newton-Raphson incremental-iterative procedure with the Newmark time integration scheme is used to obtain the non-linear transient dynamic motion of the on-board rotor. Moreover, a robust shooting algorithm is used to obtain the periodic non-linear responses of the rotor and is validated against the transient time integration algorithm.

When the rotor is excited by a sinusoidal rotation of the support, it is shown that a dynamic instability of the rotor can occur because of time-varying parametric coefficients in the linearized equations of motion and that the linearized hydrodynamic bearings have an influence greater than the rigid bearings on the size of the instability regions of the rotor.

It is noted that the shape and the magnitude of the rotor orbits can be significantly affected by the frequency and amplitude of the support motions. As expected, the linearized bearing model gives accurate results only for small amplitudes of the support motions. 
Also, this simplified bearing model cannot account for non-linear phenomena and does not permit to predict the change of dynamic regime such as period-doubling motion and transition to chaos. Conversely, these phenomena are observed by means of diagrams of bifurcation of dynamic responses when the non-linear bearing model is considered.

It is observed that depending on the amplitudes of the sinusoidal motions of the support, the non-linear rotor system can exhibit sub-harmonic, quasi-periodic and chaotic motions. Lastly, it is well demonstrated that different initial conditions for the non-linear transient dynamic motion can lead to different responses of the rotor in the presence of the support motions.

\section{Appendix A}

The analytical expressions of the components of the damping and stiffness matrices $\mathbf{c}_{\mathrm{be}}^{\mathbf{R}^{\text {be }}}$ and $\mathbf{k}_{\mathrm{be}}^{R^{\text {pe }}}$ in the polar frame linked with the bearing $R^{\text {be }}$ are given by

$$
\mathbf{c}_{\mathrm{be}}^{R^{\text {be }}}=\frac{W_{\mathrm{r}}}{c_{\mathrm{be}} \Omega}\left[\begin{array}{cc}
c_{\mathrm{be}}^{\text {tata }} & c_{\mathrm{be}}^{\text {tara }} \\
c_{\mathrm{be}}^{\text {rata }} & c_{\mathrm{be}}^{\text {rara }}
\end{array}\right] ; \quad \mathbf{k}_{\mathrm{be}}^{R^{\text {be }}}=\frac{W_{\mathrm{r}}}{c_{\mathrm{be}}}\left[\begin{array}{ll}
k_{\mathrm{be}}^{\text {tata }} & k_{\mathrm{be}}^{\text {tara }} \\
k_{\mathrm{be}}^{\text {rata }} & k_{\mathrm{be}}^{\text {rara }}
\end{array}\right]
$$

with

$$
\begin{aligned}
& c_{\mathrm{be}}^{\text {tata }}=2 k_{\mathrm{be}}^{\text {rata }} \quad ; \quad c_{\mathrm{be}}^{\text {tara }}=-2 k_{\mathrm{be}}^{\text {tata }} ; \quad c_{\mathrm{be}}^{\text {rata }}=-2 k_{\mathrm{be}}^{\text {tata }} ; c_{\mathrm{be}}^{\text {rara }}=-2 k_{\mathrm{be}}^{\text {tara }} \\
& k_{\mathrm{be}}^{\text {tata }}=4 f_{\mathrm{be}}\left(\varepsilon_{\mathrm{be}}^{0}\right) ; \quad k_{\mathrm{be}}^{\text {tara }}=\frac{-\pi\left(1+2 \varepsilon_{\mathrm{be}}^{02}\right)}{\varepsilon_{\mathrm{be}}^{0} \sqrt{1-\varepsilon_{\mathrm{be}}^{02}}} f_{\mathrm{be}}\left(\varepsilon_{\mathrm{be}}^{0}\right) ; \quad k_{\mathrm{be}}^{\text {rata }}=\frac{\pi \sqrt{1-\varepsilon_{\mathrm{be}}^{02}}}{\varepsilon_{\mathrm{be}}^{0}} f_{\mathrm{be}}\left(\varepsilon_{\mathrm{be}}^{0}\right) ; \quad k_{\mathrm{be}}^{\text {rara }}=\frac{8\left(1+\varepsilon_{\mathrm{be}}^{02}\right)}{1-\varepsilon_{\mathrm{be}}^{02}} f_{\mathrm{be}}\left(\varepsilon_{\mathrm{be}}^{0}\right) \\
& f_{\mathrm{be}}\left(\varepsilon_{\mathrm{be}}^{0}\right)=\left(16 \varepsilon_{\mathrm{be}}^{02}+\pi^{2}\left(1-\varepsilon_{\mathrm{be}}^{02}\right)\right)^{\frac{-1}{2}}
\end{aligned}
$$

\section{References}

[1] B. Xu, L. Qu, R. Sun, The optimization technique-based balancing of flexible rotors without test runs, Journal of Sound and Vibration 238 (5) (2000) 877-892.

[2] Y. Kang, T.-W. Lin, Y.-J. Chang, Y.-P. Chang, C.-C. Wang, Optimal balancing of flexible rotors by minimizing the condition number of influence coefficients, Mechanism and Machine Theory 43 (7) (2008) 891-908.

[3] M. Lalanne, G. Ferraris, Rotordynamics Prediction in Engineering, Wiley, Chichester, 1998.

[4] G. Genta, Dynamics of Rotating Systems, Springer, New York, 2005.

[5] N. Bachschmid, P. Pennacchi, E. Tanzi, Cracked Rotors, Springer, Berlin, 2010.

[6] R. Dufour, A. Berlioz, Parametric instability of a beam due to axial excitations and to boundary conditions, ASME Journal of Vibration and Acoustics 120 (2) (1998) 461-467.

[7] A. Berlioz, J. Der Hagopian, R. Dufour, E. Draoui, Dynamic behavior of a drill-string: Experimental investigation of lateral instabilities, ASME Journal of Vibration and Acoustics 118 (3) (1996) 292-298.

[8] H.D. Nelson, J.M. McVaugh, The dynamics of rotor-bearing systems using finite elements, ASME Journal of Engineering for Industry 98 (2) (1976) 593-600.

[9] Y. Kang, Y.-P. Shih, A.-C. Lee, Investigation on the steady-state responses of asymmetric rotors, ASME Journal of Vibration and Acoustics 114 (2) (1992) 194-208.

[10] A. Nandi, S. Neogy, An efficient scheme for stability analysis of finite element asymmetric rotor models in a rotating frame, Finite Elements in Analysis and Design 41 (14) (2005) 1343-1364.

[11] Y. Ishida, J. Liu, T. Inoue, A. Suzuki, Vibrations of an asymmetrical shaft with gravity and nonlinear spring sharacteristics (isolated resonances and internal resonances), ASME Journal of Vibration and Acoustics 130 (4) (2008) 041004-1 to 041004-8.

[12] M.M. Khonsari, Y.J. Chang, Stability boundary of non-linear orbits within clearance circle of journal bearings, ASME Journal of Vibration and Acoustics 115 (3) (1993) 303-307.

[13] S. Zhao, H. Xu, G. Meng, J. Zhu, Stability and response analysis of symmetrical single-disk flexible rotor-bearing system, Tribology International 38 (8) (2005) 749-756.

[14] D. Li, J. Xu, A method to determine the periodic solution of the non-linear dynamics system, Journal of Sound and Vibration 275 (1-2) (2004) 1-16.

[15] R.D. Brown, P. Addison, A.H.C. Chan, Chaos in the unbalance response of journal bearings, Nonlinear Dynamics 5 (4) (1994) $421-432$.

[16] T. Zheng, N. Hasebe, An efficient analysis of high-order dynamical system with local nonlinearity, ASME Journal of Vibration and Acoustics 121 (3) (1999) 408-416.

[17] G. Shen, Z. Xiao, W. Zhang, T. Zheng, Nonlinear behavior analysis of a rotor supported on fluid-film bearings, ASME Journal of Vibration and Acoustics 128 (1) (2006) 35-40.

[18] B. Kishor, S.K. Gupta, On the dynamic analysis of a rigid rotor-gear pair-hydrodynamic bearing system, ASME Journal of Vibration, Acoustics, Stress and Reliability in Design 111 (3) (1989) 234-240.

[19] S. Baguet, P. Velex, Influence of the nonlinear dynamic behavior of journal bearings on gear-bearing assemblies, Proceedings of the ASME 2005 International Design Engineering Technical Conferences \& Computers and Information in Engineering Conference, Long Beach, California, USA, 24-28 September 2005.

[20] S. Baguet, G. Jacquenot, Nonlinear couplings in a gear-shaft-bearing system, Mechanism and Machine Theory 45 (12) (2010) $1777-1796$.

[21] V. Srinivasan, A.H. Soni, Seismic analysis of a rotor-bearing system, Earthquake Engineering and Structural Dynamics 12 (3) (1984) 287311.

[22] B. Samali, K.B. Kim, J.N. Yang, Random vibration of rotating machines under earthquake excitations, ASCE Journal of Engineering Mechanics 112 (6) (1986) 550-565.

[23] Y. Hori, T. Kato, Earthquake-induced instability of a rotor supported by oil film bearings, ASME Journal of Vibration and Acoustics 112 (2) (1990) 160-165.

[24] L.E. Suarez, M.P. Singh, M.S. Rohanimanesh, Seismic response of rotating machines, Earthquake Engineering and Structural Dynamics 21 (1) (1992) 21-36. 
[25] R. Subbiah, R.B. Bhat, T.S. Sankar, Response of rotors subjected to random support excitations, ASME Journal of Vibration, Acoustics, Stress and Reliability in Design 107 (4) (1985) 453-459.

[26] A.S. Lee, B.O. Kim, Y.-C. Kim, A finite element transient response analysis method of a rotor-bearing system to base shock excitations using the state-space Newmark scheme and comparisons with experiments, Journal of Sound and Vibration 297 (3-5) (2006) 595-615.

[27] P. Bonello, M.J. Brennan, Modelling the dynamic behaviour of a supercritical rotor on a flexible foundation using the mechanical impedance technique, Journal of Sound and Vibration 239 (3) (2001) 445-466.

[28] F.W. da Silva Tuckmantel, K.L. Cavalca, H.F. de Castro, P. Felscher, R. Markert, An analysis on the supporting structure representative model in rotating systems, Proceedings of the Tenth International Conference on Vibration Problems, Prague, Czech Republic, September 2011.

[29] N. Feng, E. Hahn, Rotor-model-based identification of foundations in rotating machinery using modal parameters, Proceedings of the Tenth International Conference on Vibration Problems, Prague, Czech Republic, 5-8 September 2011.

[30] M. Duchemin, A. Berlioz, G. Ferraris, Dynamic behavior and stability of a rotor under base excitation, ASME Journal of Vibration and Acoustics 128 (5) (2006) 576-585.

[31] N. Driot, C.H. Lamarque, A. Berlioz, Theoretical and experimental analysis of a base-excited rotor, ASME Journal of Computational and Nonlinear Dynamics 1 (3) (2006) 257-263.

[32] F.M.A. El-Saeidy, F. Sticher, Dynamics of a rigid rotor linear/nonlinear bearings system subject to rotating unbalance and base excitations, Journal of Vibration and Control 16 (3) (2010) 403-438.

[33] A.S. Das, J.K. Dutt, K. Ray, Active vibration control of unbalanced flexible rotor-shaft systems parametrically excited due to base motion, Applied Mathematical Modelling 34 (9) (2010) 2353-2369.

[34] J. Frêne, D. Nicolas, B. Degueurce, D. Berthe, M. Godet, Hydrodynamic Lubrication Bearings and Thrust Bearings, Elsevier Science, Amsterdam, 1997.

[35] M. Dakel, S. Baguet, R. Dufour, Steady-state dynamic behavior of an on-board rotor under combined base motions, Journal of Vibration and Control, Article in press, DOI: 10.1177/1077546313483791.

[36] L.-W. Chen, D.-M. Ku, Dynamic stability of a cantilever shaft-disk system, ASME Journal of Vibration and Acoustics 114 (3) (1992) 326329.

[37] L. Peletan, S. Baguet, M. Torkhani, G. Jacquet-Richardet, A comparison of stability computational methods for periodic solution of nonlinear problems with application to rotordynamics, Nonlinear Dynamics 72 (3) (2103) 671-682.

[38] G.R. Cowper, The shear coefficient in Timoshenko's beam theory, ASME Journal of Applied Mechanics 33 (2) (1966) $335-340$. 